\title{
The Effects of Feedback Loops and Signalling Complexes in the Induction of Postsynaptic Plasticity: A Systems Biology Approach
}

\author{
by \\ Matthew MacLeod \\ A thesis submitted to the \\ Faculty of Graduate Studies and Research \\ in partial fulfillment of the requirements \\ for the degree of \\ Master of Cognitive Science \\ Institute of Cognitive Science
Carleton University
Ottawa, Ontario, Canada \\ April, 2014 \\ (C) Copyright \\ Matthew MacLeod, 2014
}




\begin{abstract}
Synaptic plasticity is the activity-dependent modification of the strength of connections between neurons, which supports learning and memory by selectively strengthening some connections while weakening others. Neural activity generates biochemical signals that activate a complex network of signalling proteins affecting synaptic change by increasing or decreasing the number of receptors present at the synapse. A key feature of this process of induction is that it requires the activation of feedback loops with sharp thresholds to sustain signalling and filter out fluctuations. Also present at the synapse are specialized proteins called scaffolds that bind members of signalling pathways and shape their dynamics in a variety of ways. Recent research has shown that scaffolds can variously strengthen and weaken the threshold properties of these pathways, depending on context. The aim of this thesis is to determine the qualitative effects of combining feedback loops and scaffold proteins in computational models of synaptic plasticity. In agreement with previous results in the literature, we found that the effect of scaffolds on threshold properties is highly dependent on biochemical context, but we did not find evidence of an interactive effect of feedback loops and scaffold proteins in this regard.
\end{abstract}




\section{Acknowledgements}

There are many people I would like to thank for their contributions and support:

First and foremost, I thank my supervisor, Dr. Anthony White. Without his insight, support, dedication, and patience, this work would not have been possible.

My family has been tremendously supportive and helpful throughout the time I have been working on this project. I thank my parents for their continued love and support. My three sisters have also been exceptionally generous with their time and advice.

I would like to thank the other members of the Complex Adaptive System Group at Carleton University for their comments and assistance over the past few years.

Special thanks to Will Hrynewich for sparking my initial interest in complex systems and engaging in endless discussions of their importance in various areas of science; he helped immensely in developing the conceptual framework that informs this work.

I thank William Hlavacek of Los Alamos National Laboratory and Leonard Harris of the University of Pittsburgh for their technical assistance in developing models with BioNetGen.

I would like to thank the Institute of Cognitive Science at Carleton University for their understanding and support.

Finally, I would like to thank my friends for understanding when I was not able to see them and, when I could, for smiling and looking interested during my many expositions on the wonders of protein interactions. 


\section{Contents}

$\begin{array}{ll}\text { Abstract } & \text { ii }\end{array}$

$\begin{array}{ll}\text { Acknowledgments } & \text { iii }\end{array}$

List of Tables vii

List of Figures

1 Introduction 1

1.1 Overview . . . . . . . . . . . . . . . . . . . 1

1.2 Problem Statement . . . . . . . . . . . . . . . . 5

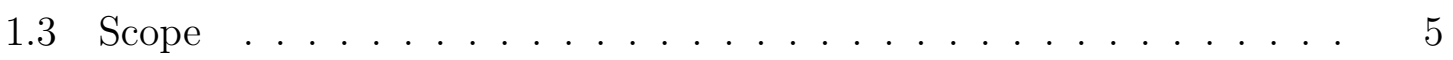

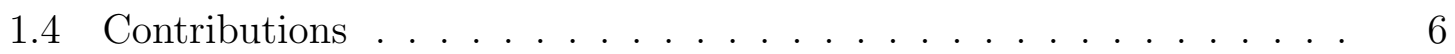

1.5 Organization of the Document . . . . . . . . . . . 7

2 Background $\quad 8$

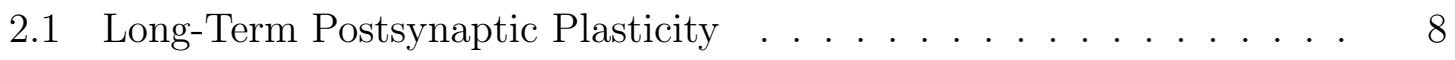

2.2 Feedback Loops . . . . . . . . . . . . . . . . . . . . . . . . 11

2.2.1 The MAPK-PLA ${ }_{2}$-PKC Positive Feedback Loop . . . . . . . 12

2.2.2 Role of the MAPK-PLA $A_{2}$-PKC Loop in the Induction of Plasticity 13

2.3 Signalling Complexes . . . . . . . . . . . . . . . . . 14

2.3.1 Mechanisms of Scaffold Protein Function . . . . . . . . . 15

2.3.2 The Role of KSR in MAPK Signalling . . . . . . . . . . 18

2.4 Systems Biology Modelling Methods . . . . . . . . . . . . . . . 19

2.4.1 Modelling Approaches . . . . . . . . . . . . . . . . . . 19 
2.4.2 The BioNetGen Modelling Framework _. . . . . . . . . 21

3 Related Work $\quad 26$

3.1 The Role of Feedback Loops in Synaptic Plasticity . . . . . . . . . 28

3.1.1 Single Feedback Loops . . . . . . . . . . . . . . . . . 28

3.1 .2 Coupled Feedback Loops . . . . . . . . . . . . . . . . 33

3.2 The Role of Scaffold Proteins in MAPK Signalling . . . . . . . . . 39

3.2 .1 Concentration Effects . . . . . . . . . . . . . . . . . . . . 39

3.2 .2 Regulatory Roles . . . . . . . . . . . . . . . . . 41

3.2 .3 Thresholding and Bistability . . . . . . . . . . . . . . 44

3.2 .4 Limitations of Scaffold Models . . . . . . . . . . . . . . . 46

3.3 Trends in Biological Modelling . . . . . . . . . . . . . . . 47

3.3.1 Approximations for Enzymatic Reactions . . . . . . . . . 47

3.4 Summary of Related Work . . . . . . . . . . . . . . . . . . . 49

4 Materials and Methods $\quad 51$

4.1 Design . . . . . . . . . . . . . . . . . . . 52

4.1.1 Application of the Systems Biology Approach . . . . . . . 52

4.1 .2 Model Selection . . . . . . . . . . . . . . . . . . . 54

4.1 .3 Experimental Design . . . . . . . . . . . . . . . 55

4.2 Implementation . . . . . . . . . . . . . . . . . 56

4.2.1 Implementation of the Synaptic Model . . . . . . . . . . 57

4.2.2 Implementation of the Scaffold Model . . . . . . . . . . . . 61

4.2 .3 Creation of Final Models . . . . . . . . . . . . . . . 63

5 Results and Discussion $\quad 65$

5.1 Results . . . . . . . . . . . . . . . . . 66

5.1.1 Basic Behaviour of the Scaffold Model . . . . . . . . . 66

5.1.2 Exploration of the Network Model . . . . . . . . . . . . 68

5.1.3 Comparison of the Pathway and Network Models . . . . . . 74

5.2 Discussion . . . . . . . . . . . . . . . . . . 78

6 Conclusions and Future Work $\quad 81$

6.1 Conclusion . . . . . . . . . . . . . . . . . 81 
6.2 Summary of Contributions . . . . . . . . . . . . . . . . . . 82

6.3 Future Work . . . . . . . . . . . . . . . . 83

6.3.1 Continuing the Search for a Feedback-Scaffolding Interaction . 83

6.3.2 Determining the Differences Between the Pathway and Network Model Responses . . . . . . . . . . . . . . . . . . . . . . 84

6.3.3 Extending the Models and Methods . . . . . . . . . . . . . . . 85

$\begin{array}{lr}\text { References } & 87\end{array}$

$\begin{array}{ll}\text { A Validation of the Synaptic Model } & 97\end{array}$

$\begin{array}{ll}\text { B Supplementary Experimentation } & 100\end{array}$ 


\section{List of Tables}

5.1 Parameters and ranges for all experiments on the pathway and network models. The only parameter that differs between the pathway and network models is the stimulus, where the pathway model uses a ratebased constant stimulus and the network model uses a concentrationbased 1-second pulse of $\mathrm{Ca}^{2+}$. Scaffold dissociation rates are the same for all kinases (Raf, MEK, and ERK). The Feedback Rate parameter range listed applies to all feedback loops in both models, although the reaction to which it is applied differs; specific reactions are provided in Section 4.1.3. Phosphatase concentrations apply to all phosphatases in both models (PP2A, PP5, and MKP), although PP1 is present only in the network model. . . . . . . . . . . . . . . . . . .

B.1 Parameter ranges for additional experiments on the pathway and network models. . . . . . . . . . . . . . . . . . . . . . . . . . . . 101 


\section{List of Figures}

2.1 Block diagram of the MAPK-PLA ${ }_{2}$-PKC positive feedback loop. The core components are shown in magenta (MAPK cascade), green $\left(\mathrm{PLA}_{2}\right)$, and cyan $(\mathrm{PKC})$. The connections comprising the loop are shown in red. 12

3.1 Single feedback loop model used by Ogasawara and Kawato in [73]. . 35

3.2 Coupled feedback loop model used by Ogasawara and Kawato in [73]. 35

3.3 Tristable synaptic system. CaMKII and PP2A each have autoactivation loops and inhibitory connections between them. Adapted from

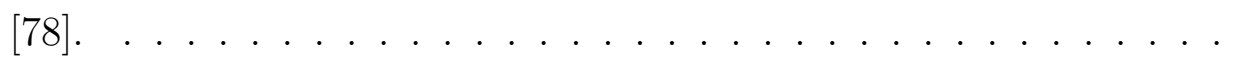

4.1 Block diagram of the synaptic signal transduction model, based on [3]

4.2 Examples of protein representation: comparison of the graphical representation from the SMART database and its corresponding representation in BNGL for the enzymes ERK (part A) and PKC (part

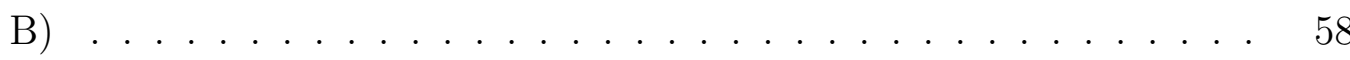

4.3 Block diagram of the core scaffold model, based on [49] . . . . . . 61

4.4 Three versions of the pathway model: A) no feedback, B) positive feedback, and C) negative feedback . . . . . . . . . . .

4.5 Three versions of the network model: A) no added feedback, B) positive feedback, and C) negative feedback . . . . . . . . . . . . 64

5.1 Effects of signal magnitude variation. Part A: Original model. Part B: Our implementation. . . . . . . . . . . . . . . . . . 66

5.2 Effects of scaffold concentration variation. Part A: Original model. Part B: Our implementation. . . . . . . . . . . . . . 66 
5.3 Results of calibration tests on the network model. Increasing scaffold dissociation rate constant restored the sigmoidal curve, while increasing the activation threshold and decreasing the maximal response. . .

5.4 Results of $\mathrm{Ca}^{2+}$ stimulus versus feedback strength for the cytoplasmic and scaffold versions of the network model. Part A shows the results with no added feedback; part B with added positive feedback; and part $\mathrm{C}$ with added negative feedback. . . . . . . . . . . . . . . 71

5.5 Results of $\mathrm{Ca}^{2+}$ stimulus versus scaffold affinity experiments for the network model under the three feedback conditions. . . . . . . . . . .

5.6 Results of feedback strength versus scaffold affinity experiments on the network model under the three feedback conditions. In part $\mathrm{A}$, an intermediate $\mathrm{Ca}^{2+}$ stimulus of $2.5 \mu \mathrm{M}$ was applied, while a strong 5.0 $\mu \mathrm{M}$ stimulus was used in part B.

5.7 Results of an example phosphatase concentration versus scaffold affinity experiment. . . . . . . . . . . . . . . . 73

5.8 Results of input stimulus versus feedback strength for the cytoplasmic and scaffold versions of the pathway model. Parts A and B show the positive and negative feedback conditions, respectively. . . . . . . . .

5.9 Results of input stimulus versus scaffold affinity experiments for the pathway model under the three feedback conditions. . . . . . . . . .

5.10 Results of feedback strength versus scaffold affinity experiments for the pathway model under positive and negative feedback conditions. In part A, a moderate stimulus of $1.0 \mathrm{~s}^{-1}$ was applied, while part B

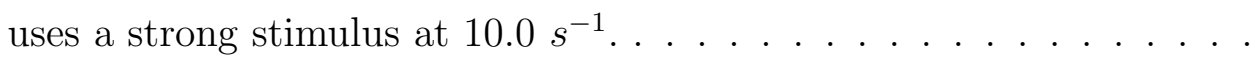

6.1 A possible sequence of steps to convert the pathway model into the network model. . . . . . . . . . . . . . . . . . .

A.1 Mean number of AMPA receptors in the synapse following stimulation with 1-second pulses of $\mathrm{Ca}^{2+}$ at different concentrations . . . . . . . .

A.2 Sigmoidal relationships between concentration of calcium stimulus and magnitude of LTD. Curves were obtained by nonlinear regression to Equation A.1 . . . . . . . . . . . . . . . . 
A.3 Estimated values of $K$ (the $\mathrm{Ca}^{2+}$ input required for induction of LTD) at different durations of stimulation . . . . . . . . . . . .

A.4 Estimated values of $n$ (the Hill coefficient) at different durations of stimulation . . . . . . . . . . . . . . . .

A.5 Estimated values of $L T D_{\max }$ (the maximum magnitude of LTD) at different durations of stimulation $\ldots \ldots \ldots \ldots$

B.1 Summary charts for supplementary experiments on the pathway model without feedback. . . . . . . . . . . . . . . . . . . . . . . 101

B.2 Summary charts for supplementary experiments on the pathway model with positive feedback. . . . . . . . . . . . . . . . . . . 101

B.3 Summary charts for supplementary experiments on the pathway model with negative feedback. . . . . . . . . . . . . . . . . . 102

B.4 Summary charts for supplementary experiments on the network model default feedback. . . . . . . . . . . . . . . . . . 102

B.5 Summary charts for supplementary experiments on the network model with positive feedback. . . . . . . . . . . . . . . . 102

B.6 Summary charts for supplementary experiments on the network model with negative feedback. . . . . . . . . . . . . . . . . 102 


\section{Chapter 1}

\section{Introduction}

\section{$1.1 \quad$ Overview}

Synaptic plasticity is the activity-dependent modification of the strength of connections between neurons, and has been proposed as a cellular mechanism underlying learning and memory [9]. In the last few decades, the volume of research on synaptic plasticity has dramatically increased, producing many significant advances. From the humble beginnings of a single mechanism identified at specific hippocampal synapses in the 1970s, we know of a wide variety of plasticity forms that operate over time scales from milliseconds to weeks, in both strengthening and weakening modes, on both sides of the synapse, and in most (if not all) areas of the brain [24, 46, 67]. Despite this staggering diversity, all forms of synaptic plasticity seem to proceed through

a series of stages, including induction, expression, and maintenance [1]. The majority of research in this area has focused on long-term postsynaptic forms of plasticity, known as long-term potentiation (LTP) and long-term depression (LTD). Researchers have recently made great strides in elucidating the molecular underpinnings of LTP 
and LTD, which take the form of signal transduction pathways that link together to form complex biochemical networks [9].

One of the key features of signal transduction networks is that they make extensive use of feedback loops as control structures [17]. Feedback loops have long been central to computational models of synaptic plasticity because of their tendency to form switching elements capable of controlling the induction of different forms of plasticity [33]. In postsynaptic plasticity, induction is the phase during which incoming action potentials are converted into biochemical activity that activates either LTP or LTD [1]. Many feedback loops have been proposed to play a role in induction, some of which have been studied with both experimental and computational methods [9]. In particular, positive feedback loops have been shown to produce a sharp threshold in terms of input stimuli which allows specific stimulus magnitudes to induce different forms of plasticity $[12,44,78]$. Overall, research in this area has made considerable progress in identifying the enzymes involved in synaptic feedback loops and establishing the role of feedback in the induction of plasticity.

In addition to the signalling enzymes that enact the biochemical changes involved LTP and LTD, a variety of other proteins, known as scaffold proteins, are important participants in synaptic plasticity [23]. On the inside surface of the postsynaptic membrane, scaffolds link together with cytoskeletal proteins to form an intricate structure known as the postsynaptic density (PSD) which serves to bring receptors, enzymes, and other signalling molecules together in space and time $[16,75]$. In particular, scaffold proteins are thought to contribute to the organization of signalling reactions by binding several members of a pathway in order to facilitate their interactions [32]. The resulting structures, known as signalling complexes, have been shown to have a 
variety of effects on spatial and temporal dynamics of signal transduction, including enhancements in the speed, specificity, and diversity of responses [85].

While there have been many computational modelling studies focusing on the signalling network involved in postsynaptic plasticity, no known work has considered the role of signalling complexes. There are likely two reasons for this situation. Firstly, of the many different kinds of scaffolds that have been identified in the PSD, relatively few have well-characterized roles in synaptic function [83]; even among those that are well-characterized, the kinetic parameters required to build a detailed model are available for only a subset of the known interactions. Secondly, and probably more importantly, signalling complexes are notoriously difficult to model because they can exist in a large number of states. This problem, known as combinatorial complexity, occurs because of the many ways in which proteins can be combined and modified [34]. As part of their function, enzymes often chemically modify target proteins by adding small molecules (such as phosphates) to specific sites. A given protein may have many such modification sites, each of which can be in two states. Thus, a protein with $n$ modification sites can exist in $2^{n}$ distinct states. Moreover, when we consider binding interactions among proteins, the number of potential states of the resulting complexes makes it extremely challenging to write out all the reactions in order to build a model. Recently, however, new modelling methods have been developed in the field of systems biology that are specifically designed to deal with the combinatorial complexity of signalling systems [14].

Systems biology is a rapidly advancing interdisciplinary field of biological research that emphasizes the importance of the emergence of system-level behaviour through the interaction of components across spatiotemporal scales $[47,50,69]$. The devel- 
opment of systems biology was mainly a reaction to reductive approaches that aim to explain biological phenomena by reference to the function of a small set of fundamental components, typically genes; in systems biology, by contrast, the interactions among components are taken to be at least as important as their individual functions in the generation of high-level behaviour [69]. The rise of systems biology has been swift and decisive, largely due to the advent of genome sequencing and other high-throughput techniques in biochemistry. These methods have generated unprecedented quantities of data about the basic building blocks of biological systems without providing a framework for integrating those data and understanding how the building blocks work together $[39,47,50,69]$. Much as a list of roads, intersections, and cars does little to explain the dynamics of traffic flow in a city, the identification of the parts of a cell is insufficient to explain its function. Of course, the identification of the parts is an essential first step, but to understand how those parts work together, we must construct models and conduct simulations [47, 69].

While synaptic models have yet to consider signalling complexes, researchers outside of computational neuroscience have recently begun to build models of scaffold proteins and study their effects in signal transduction. Work in this area is at an early stage, but several studies have demonstrated important functions of scaffolds, including regulation of the speed, strength, and selectivity of signalling responses $[59,60]$. To date, all of these models have focused on the role of scaffolds in the mitogen-activated protein kinase (MAPK) cascade, which is involved in a variety of cellular functions (including synaptic plasticity) and is well-known for having a sharp activation threshold that produces a switch-like response [21, 52]. One of the key issues in this field is that there are conflicting reports concerning the effects of scaffolds 
on the threshold properties of the MAPK cascade: some suggest that scaffolds tend to soften the threshold, making the response more graded and less switch-like [57, 49], while others conclude that either case is possible depending on the parameters and/or reaction mechanisms used in the model $[20,93]$. In light of the properties of feedback loops described above, it is note-worthy that none of these scaffold models have taken the effects of feedback into consideration [95]. Thus, the role of feedback loops in models of scaffold-mediated signalling is an important unanswered question.

\subsection{Problem Statement}

Models of postsynaptic plasticity have focused on the role of feedback loops in defining thresholds for the induction of LTP and LTD, but they have not considered the effects of scaffold proteins in this process. Models of signalling complexes disagree about the effects of scaffold proteins on the activation threshold in MAPK signalling, but they have not considered the role of feedback loops in this process. This situation provides an opportunity for cross-fertilization between these two fields. Therefore, our problem statement is: to determine the qualitative effects feedback loops and signalling complexes on the thresholding properties displayed in the induction of synaptic plasticity.

\subsection{Scope}

In order to address our problem statement, we extended previously published models in the fields of scaffold-mediated signalling and synaptic plasticity. Specifically, we extended a scaffold model [49] by adding feedback loops and we extended a synaptic plasticity model [3] by adding a scaffold protein. Since both of these models include 
the MAPK cascade, we were able to incorporate the scaffold considered in [49] into our implementation of the synaptic model. We added the same feedback loops (one positive and one negative) to both models and developed versions with and without scaffolds, which yielded a set of models with different combinations of feedback and scaffolding. We conducted simulation experiments on these models using a variety stimulus conditions and parameter ranges. All of these experiments used deterministic simulation methods. Our analysis was qualitative in nature and consisted of observing differences in plots of the experimental results. We did not perform statistical analyses on our results.

\subsection{Contributions}

The primary contributions made by this thesis are as follows:

- Synthesis and extension of existing models using recently developed methods (Section 4.2)

- Development of flexible domain-oriented representations for all proteins involved (Section 4.2.1)

- Confirmation of the basic effects of scaffolds in MAPK signalling (Section 5.1.1)

- Further investigation into the complex effects of scaffolds on thresholds (Sections 5.1 .2 and 5.1.3)

- A first examination of the effects of feedback loops and signalling complexes in the same model (Sections 5.1.2 and 5.1.3) 


\subsection{Organization of the Document}

In Chapter 2, we provide background material on the core elements of our work: synaptic plasticity, feedback loops, signalling complexes, and systems biology. Chapter 3 presents a selection of previous research involving computational modelling of synaptic plasticity and scaffold-mediated signalling. Chapter 4 describes the selection and implementation of the models used in this work, as well as the simulations carried out to address the problem statement. In Chapter 5, we present the results of our research and discuss how they related to those of previous studies. Finally, in

Chapter 6, we discuss our conclusions and provide some possible directions for future work. 


\section{Chapter 2}

\section{Background}

In this chapter, we provide background material pertinent to the research presented in this thesis. Since our topic is the application of systems biology approaches to modelling feedback loops and signalling complexes in the induction of long-term postsynaptic plasticity, we describe each of these elements in the sections below. In Section 2.1, we outline the functional phases of postsynaptic plasticity, known as induction, expression, and maintenance. Sections 2.2 and 2.3 present the properties and functions of feedback loops and signalling complexes, respectively, and place special em-

phasis on the loops and complexes featured in our models. Finally, in Section 2.4, we review the modelling approaches used in systems biology and introduce BioNetGen, the framework we used to develop the models presented in this thesis.

\subsection{Long-Term Postsynaptic Plasticity}

At the most basic level, synaptic plasticity refers to the set of processes that modify the strength of connections between neurons. In long-term postsynaptic plasticity, the 
primary mechanism of affecting the strength of a synapse is by changing the number of AMPA receptors (AMPARs) embedded in the dendritic membrane [24]. AMPARs are activated by the excitatory neurotransmitter glutamate and are largely responsible for generating the electrochemical potentials that drive neural firing. Therefore, increasing the number of AMPARs strengthens the connection, known as long-term potentiation (LTP), and decreasing the number weakens it, known as long-term depression (LTD) [62]. The biochemical processes underlying the insertion and removal of AMPARs from the dendritic membrane have been extensively studied to reveal an intricate signal transduction network at the heart of synaptic plasticity [1]. In general, a signal transduction network consists of a set of proteins (mostly enzymes) that receive signals from receptors, integrate them, and initiate a cellular response. Correspondingly, the processes underlying synaptic plasticity can be divided into three broad phases: induction, expression, and maintenance [1, 24, 62].

Induction is the process of converting incoming stimuli into the intracellular biochemical signals that trigger different forms of plasticity [1]. Briefly, glutamate released from the axonal terminal binds to the NMDA receptors in the dendritic membrane, causing an influx of calcium ions. Once inside the dendritic spine, $\mathrm{Ca}^{2+}$ ions activate a variety of target proteins and enzymes. LTP and LTD are both triggered by $\mathrm{Ca}^{2+}$ influx, but the the amplitude and frequency of input determines which form is induced. Strong and rapid input induces LTP, while slower and more moderate input induces LTD. Distinct downstream effectors (primarily kinases and phosphatases) are activated by these input profiles such that their selective activation allows for the induction of one type of plasticity over the other $[1,62]$. Finally, feedback loops are also important for induction. Because intracellular calcium signals tend to be short- 
lived (typically lasting a second or less), the activation of a positive feedback loop can sustain signalling long enough to affect the required changes. Moreover, positive feedback can sharpen the threshold $\mathrm{Ca}^{2+}$ input required for induction, making the process more switch-like (see Section 2.2) [90].

In the expression phase of synaptic plasticity, the enzymes activated during induction enact short- and long-term changes in synaptic strength [24]. The short-term changes involve modification of receptors that are already embedded in the dendritic membrane. For instance, phosphorylation of AMPARs by PKC and CaMKII temporarily enhances ion flow, leading to stronger postsynaptic potentials; other receptors and ion channels are similarly modified to influence their biophysical properties, such as conductivity, affinity for agonists, and gating kinetics $[1,62]$. On longer time scales, receptors cycle between the dendritic membrane and internal vesicular pools. During LTP, phosphorylation of AMPARs by PKA drives their insertion into the membrane, whereas dephosphorylation by calcineurin triggers their removal during LTD Ajay2006. Finally, synaptic changes can also be expressed through changes in the shape and size of the dendritic spine $[1,24,62]$.

The lifetime of expressed synaptic changes is limited by molecular turnover (loss of proteins through degradation or diffusion away from the synapse) and by stochastic noise [1]. In both cases, the feedback loops activated during induction have been postulated to play a mitigating role $[1,33,71,88]$. Proteins lost in turnover can be replaced by new proteins synthesized locally in the dendrites, but they arrive at the synapse in a 'naive' state that may not match the currently expressed form of plasticity. One proposed mechanism to solve this problem is that the feedback loops activated during induction form bistable switches that can remain persistently active 
(see Section 2.2.2) [1,33]. Replacement proteins can then recruited to the current state of the synapse because the enzymes required to modify them are still active. Experimental and computational studies have both shown that the time scales of new protein synthesis and sustained feedback signalling are consistent with this proposal: synthesis requires approximately an hour and feedback can sustain signalling for at least that long $[74,90]$. Stochasticity poses a challenge for the maintenance of synaptic changes because dendritic spines have small volumes (typically around $0.1 \mathrm{fL}$ ) and often contain only a few copies of key molecules, which can cause substantial fluctuations in signalling reactions. Positive feedback loops have been proposed provide a mechanism capable of filtering out stochastic fluctuations while still responding to sufficiently strong stimuli $[71,87,88]$. This topic is discussed in more detail in Sections 3.1.1 and 3.1.2.

\section{$2.2 \quad$ Feedback Loops}

Positive and negative feedback mechanisms that return output signals back to their inputs are ubiquitous control structures in biochemical networks [17]. In general, positive feedback can produce bistability and switch-like behaviour, whereas negative feedback tends to produce stability and adaptation [48]. These simple functions become much more complex when we consider that both types of feedback typically involve loops that depend on many components and that the arrangement and kinetics of the component proteins can greatly impact the overall function performed by the loop. Thus, depending on the configuration and characteristics of the components, positive feedback loops can act as amplifiers, accelerators/delays, or bistable switches, and negative feedback loops can act as homeostatic regulators, output limiters, adap- 
tive filters, and transient signal generators [17]. Different forms of feedback loops can be coupled to produce a wide variety of dynamic spatial and temporal patterns, increasing the complexity again $[17,40,79]$.

While several feedback loops have been described in the synaptic plasticity literature, we focus on a positive feedback loop involving the mitogen-activated protein kinase (MAPK) cascade, phosopholipase $\mathrm{A}_{2}\left(\mathrm{PLA}_{2}\right)$, and protein kinase $\mathrm{C}(\mathrm{PKC})$. Since it was first proposed by Bhalla and Iyengar in 1999 [10], the MAPK-PLA ${ }_{2}^{-}$ PKC loop (MPPL) has featured prominently in modelling studies of the induction of synaptic plasticity; it is also the primary feedback loop in the models presented in this thesis. In the following sections, we outline the biochemistry of the components of the MPPL (Section 2.2.1) and describe its roles in induction (Section 2.2.2).

\subsubsection{The MAPK-PLA ${ }_{2}$-PKC Positive Feedback Loop}

Figure 2.1 depicts the structure of the MPPL and highlights its core components. The MAPK cascade (shown in magenta) is an evolutionarily conserved signalling pathway of three enzymes activated in sequence; it is found in virtually all eukaryotes and is involved in the regulation of many cellular functions, including gene expression, the cell cycle, proliferation, differentiation, stress signalling, and apoptosis [21, 37, 80]. The first enzyme in the cascade is the kinase Raf, which is activated by cell surface receptors, GTPase binding, and phosphorylation by other enzymes [55]; these activators are collectively represented by the 'Raf-act' block in Figure 2.1. Once activated, Raf phosphorylates and activates MAPK/ERK kinase (MEK), which then goes on to phosphorylate and activate extracellular signal-regulated kinase (ERK), the final enzyme in the cascade $[21,37,80]$. The activation of the MAPK cascade contributes to 




Figure 2.1: Block diagram of the MAPK-PLA $\mathrm{M}_{2}$ PKC positive feedback loop. The core components are shown in magenta (MAPK cascade), green $\left(\mathrm{PLA}_{2}\right)$, and cyan (PKC). The connections comprising the loop are shown in red.

the activation of $\mathrm{PLA}_{2}$ through phosphorylation by ERK. PLA 2 is also activated by the binding of $\mathrm{Ca}^{2+}$, which stimulates its translocation to the cell membrane, where it catalyzes the production of arachidonic acid (AA) from phospholipid precursors [19]. AA acts synergistically with $\mathrm{Ca}^{2+}$ and diacylglycerol (DAG) to activate PKC and stimulate its translocation to the membrane [68]. Once at the membrane, PKC contributes to the activation of Raf by phosphorylating one or more of Raf's upstream activators (Raf-act in Figure 2.1) [3]. The activation of Raf by PKC closes the loop, completing the MPPL. 


\subsubsection{Role of the MAPK-PLA $A_{2}$-PKC Loop in the Induction of Plasticity}

The primary function of the MPPL in the induction of synaptic plasticity is to amplify and sustain signalling by acting as a bistable switch [90]. There are several mechanisms at work here. The sequential activation of enzymes in the MAPK cascade leads to dramatic amplification of signalling because each activated enzyme can activate many of its targets [52]. Another key feature of the MAPK cascade is that MEK and ERK each require two phosphorylation events to become active; this multistep activation leads to a property called ultrasensitivity, which filters out small stimuli to produce a sharp activation threshold and allow the system to respond in a switch-like

manner $[15,30]$. This effect is strongest when the enzyme and substrate fully dissociate between phosphorylation reactions, a reaction mechanism known as distributive catalysis; alternatively, processive catalysis occurs when the enzyme and substrate remain bound between phosphorylation reactions, which tends to produce graded responses that are proportional to the stimulus magnitude [15, 29, 30]. Both MEK and ERK are known to activated through distributive catalysis, which contributes to the sharp response thresholds they display $[52,64]$. Moreover, because the MPPL is a positive feedback loop, it has the potential to create a bistable switch. Bistability is particularly important for cellular decision-making because it allows the system to exist in two stable states, one of low activity and the other of high activity, separated by an unstable state that corresponds to the stimulus threshold required to move the system from one state to the other [30]. The key feature of bistable switches is that they produce an all-or-none response to stimuli $[15,17,30]$, and this makes them ideal for situations in which the cell requires a binary 'yes-or-no' response. Taken together, 
these mechanisms allow the MPPL to respond strongly to stimuli that exceed the activation threshold and to sustain this response for a period of hours [90].

\subsection{Signalling Complexes}

One of the intriguing features of signal transduction is that signalling pathways are assembled from a common set of proteins and enzymes, yet they are still able to respond to and propagate distinct signals [85]. Over the past few decades, a large body of research has focused on this conundrum and the solution seems to be that separate pathways are organized into modules known as signalling complexes. The key feature of signalling complexes is that they are assembled around specialized scaffold proteins that selectively bind the members of a given pathway, thereby providing an additional layer regulation and control in signal transduction [18, 38, 65, 85]. In the following sections, we describe the basic mechanisms of scaffold function (Section 2.3.1) and discuss the role of the scaffold KSR in the MAPK cascade (Section 2.3.2).

\subsubsection{Mechanisms of Scaffold Protein Function}

A scaffold is defined as a protein that binds two or more components of a signalling pathway and facilitates their interaction $[32,97]$. Scaffolds typically do not have any intrinsic catalytic activity and function solely through protein interaction domains and binding motifs, although so-called 'novel' scaffolds have recently been described that do have functional catalytic domains [2]. Here, we focus on the former category (the 'classical' scaffolds) and describe the mechanisms through which they contribute to the regulation signal transduction networks. These mechanisms can be divided 
into four categories: enforced proximity, combinatorial assembly, dynamic regulation, and conformational fine-tuning [97].

Perhaps the most basic mechanism of scaffold function is enforced proximity, which can enhance the speed and specificity of signalling reactions simply by tethering pathway members in place $[32,97]$. When an enzyme and its substrate are bound to a scaffold, their effective concentrations are increased because diffusion is no longer a limiting factor; therefore, the reaction can occur more quickly and efficiently than it would if the enzyme and substrate had to meet, bind, and react in the cytoplasm $[18,97]$. Furthermore, most signalling proteins participate in many interactions, which can potentially lead to excessive crosstalk between pathways and compromise the specificity of signalling. Scaffolds can help solve this problem by selectively binding pathway members and restricting their action to a subset of their interaction partners [85]. In addition to assembling the members of a pathway, scaffolds are often localized to specific regions of the cell and this further enhances signalling specificity by spatially segregating pathways $[32,85]$.

Scaffolds often interact with a variety of different proteins that bind to the scaffold independently, generating a (potentially very large) set of complexes with different complements of components. From a small set of building blocks, this combinatorial assembly process can create heterogeneous signalling complexes that are capable of producing distinct responses to the same input signal or, conversely, selectively responding to different types of input signal $[18,38,85]$. Moreover, many scaffolds bind both positive and negative regulators of a pathway, which can generate complex temporal response patterns $[32,85,97]$. Thus, through the combinatorial assembly of signalling complexes, scaffolds contribute to the diversity of responses a pathway 
is capable of producing.

Another key feature of scaffolds is that they enable dynamic, stimulus-dependent assembly and disassembly of signalling complexes $[18,85]$. A common mechanism for dynamic regulation involves phosphorylation of one of the scaffolds's binding domains. In some cases, this is a prerequisite for recruiting an enzyme; in other contexts, phosphorylation prevents enzymes from binding and may also dislodge bound proteins $[2,32,97]$. These regulatory phosphorylation events are often coupled with translocation events that may either move the scaffold to or away from its binding partners, depending on the context and particular scaffold [85, 97]. Scaffolds are also regulated in terms of concentration through synthesis of new proteins and targeted degradation of existing ones $[32,85]$. All of these dynamic regulation mechanisms allow scaffolds to control the overall activity of a pathway, either in a graded fashion or by activating/deactivating it altogether [97].

Finally, some scaffolds exert a more direct influence on bound enzymes by inducing conformational change in the enzyme that affects its function [2]. Also known as allosteric regulation, this type of conformational fine-tuning can bidirectionally modify enzyme activity through a number of mechanisms. In some cases, the conformational change exposes (or conceals) a binding domain or phosphorylation site on the enzyme that is crucial for its interactions; in other cases, the catalytic activity of the enzyme is modified $[2,32,97]$. Allosteric regulation is highly complex because its outcome depends on the three-dimensional structure of the enzyme and the precise distribution of electrochemical properties within that structure $[27,86]$. Conformational changes can also affect signalling dynamics through cooperative binding, in which the binding of one pathway member makes it more or less favourable energetically for other 
members to bind; these effects often depend on a specific ordering of binding events $[2]$.

The mechanisms of scaffold function discussed above can be classified according to whether or not they involve an 'active' influence or role of the scaffold on its associated pathway(s) [2, 97]. Enforced proximity and combinatorial assembly are considered to be passive mechanisms because their effects depend solely on the structure of scaffolds and the physical constraints imposed by that structure [97]. The increases in signalling efficiency and specificity provided by enforced proximity emerge from the simple fact that the pathway components are all tethered together in a small spatial volume; likewise, the diversity of responses generated through combinatorial assembly result from the fact that scaffolds are composed of modular domains to which proteins can bind independently [97]. In contrast, dynamic regulation and conformational fine-tuning involve more complex interactions between the scaffold and its partners through which the intrinsic activities of one or both are altered $[2,97]$. Now in reality, scaffolds are likely free to employ any combination of these mechanisms; for the purposes of understanding how the mechanisms differ, however, the active-passive distinction is a useful one.

\subsubsection{The Role of KSR in MAPK Signalling}

Kinase Suppressor of Ras (KSR) is a scaffold protein associated with the MAPK cascade in mammals and it is the primary MAPK scaffold expressed in neural tissue [95]; it is also the focus of the scaffold modelling studies reviewed in Section 3.2 as well as the scaffold featured in the models presented in this thesis. Moreover, KSR makes a good case study because it displays all of the mechanisms outlined 
in the previous section. Structurally, KSR is composed of five conserved domains that contain the binding sites for Raf, MEK, and ERK, along with a phospholipid binding domain and a so-called 'pseudokinase' domain that is structurally related to Raf's catalytic domain but lacks intrinsic catalytic activity [52]. KSR binds all three members of the MAPK cascade: Raf only binds when activated; MEK binds constitutively (i.e., independent of activation state); and ERK primarily binds when inactivated (although it does bind weakly when active) [49, 95]. The regulation of KSR involves both phosphorylation events and translocation events. When the cell is at rest, IMP phosphorylates KSR is on two serine residues that form binding sites for 14-3-3 proteins that restrict KSR to the cytoplasm. Stimulation of growth factor receptors leads to activation of $\mathrm{PP} 2 \mathrm{~A}$, which dephosphorylates the serine residues and releases KSR to translocate to the cell membrane where it is placed in close proximity to Raf (which is also activated by the growth factor stimulus $[52,95]$. In addition, there is evidence that high concentrations of activated ERK can phosphorylate KSR at a site close to the Raf binding site, which disrupts Raf binding and releases KSR from the membrane [66]. Finally, recent research has shown that the binding of KSR and Raf involves the pseudokinase of KSR, which allosterically regulates and enhances the catalytic activity of Raf [36].

\subsection{Systems Biology Modelling Methods}

One of the key features of systems biology is its reliance on computational models to explore the properties of biological systems and generate predictions that can be experimentally tested [47]. A variety of methods have been developed in this regard. Here, we review the major modelling approaches used and introduce the 
modelling framework used to develop the models presented in this thesis, which is called BioNetGen.

\subsubsection{Modelling Approaches}

In terms of computational modelling, the systems biology approach combines elements from more traditional bottom-up and top-down approaches [50]. The bottom-up approach begins with the most basic components and attempts to reconstruct the system, piece by piece and level by level; it is an integrative approach in which the parts are assembled so that the behaviour of the system emerges naturally through their interactions. The issue with this approach is that biological systems are so richly structured that they provide a incredibly large supply of parts and it is difficult to know where to start and stop the process of adding detail $[6,50]$. The top-down approach works in the other direction, beginning with the known high-level behaviour of the system and attempting to identify the lower-level components whose function can account for this behaviour; it is a reductive approach in which behaviour is located in a small set of key parts that drive the system. The issue with this approach is that the components of biological systems tend to be strongly interdependent and it is easy to discount interactions that are critical for the emergence of the behaviour of interest $[50,69]$.

The dominant modelling approach in systems biology combines the bottom-up and top-down approaches to produce the so-called 'middle-out' approach. Noble and colleagues clearly define the basic process:

Modeling begins at any level of organization at which there are sufficient reliable data to build a model. This is the starting point of the middle-out approach. It involves exploration of parameter spaces at the chosen level. 
The next step is to reach toward both higher and lower levels of structural complexity (the "out" part of the metaphor) [50].

This quotation exemplifies the pragmatic character of the systems biology approach: the starting point is determined by the available data rather than predefined conceptions about the 'true' or 'best' level at which to begin. Indeed, this pragmatism is motivated by the recognition that the functional and organizational levels of biological systems are deeply intertwined; this same recognition also motivates the requirement for including levels above and below the chosen middle, because the notion that biological function emerges through the bidirectional flow of information between levels is fundamental in systems biology $[69,70,77]$. In short, the middle-out approach locally applies integration and reduction, adding detail where needed to support known interactions, until the model can reliably produce the properties or behaviours of interest [50]. Another way to express the spirit of the systems biology approach is to say that it attempts to strike a balance between Occam's razor - the well-known principle that assumptions should not be multiplied beyond necessity — and Occam's broom - the more recent principle, attributed to Sydney Brenner [81], that facts are often swept under the rug because they are difficult or inconvenient.

\subsubsection{The BioNetGen Modelling Framework}

A wide variety of modelling frameworks have been applied in computational systems biology research. In accordance with the pragmatic approach described in the previous chapter, the choice of a modelling framework is determined by the system under

study, the research question, and the available data [61]. However, a recent trend in the field is to classify frameworks according to the distinction between mathematical 
and computational models. In their seminal paper on the topic, Fisher and Henzinger [31] describe this distinction as follows. Mathematical models are expressed in equations that are designed to calculate the current state of the system based on its previous state; they describe relationships among quantities and define how they change over time. In contrast, computational models are expressed in rules that are designed to generate the behaviour of the system by imitating the operation of its parts; they describe relationships among objects and define how they react to each other in time [31]. In this section, we introduce BioNetGen as a well-established systems biology modelling framework that blends mathematical and computational elements.

The distinguishing features of BioNetGen are its use of graphs to represent the domain structure of proteins and its use of graph rewriting rules to define potentially large classes of reactions that are possible in the system. Models are specified using the simple but powerful BioNetGen modelling language, known as BNGL. Once a model is written, the software applies the reaction rules to the initial species to generate all possible species and reactions that can occur in the system. The reaction network output by this process can then be simulated using deterministic (ODEs) or stochastic methods (the Gillespie Algorithm). [28]. In this way, BioNetGen combines elements of mathematical and computational approaches.

A BioNetGen model has four basic parts: parameters, molecule types, reaction rules, and output functions [14]. The parameters section defines rate constants for reactions and initial concentrations for the chemical species that seed the simulation. The molecule types section specifies the basic types of molecules in terms of their internal components and states. The reaction rules section defines the classes of 
chemical reactions that can occur in the system. Finally, the output functions section contains calls to BioNetGen's built-in functions for network generation and simulation [14]. Here, we focus on the definition of molecule types and reaction rules.

The first step in building a BioNetGen model is to develop representations for each of the basic molecule types. Each molecule type has a number of components, which may represent structural features, binding sites, or phosphorylation sites. Components can have states (a phosphorylation site could have a phophorylated and unphophorylated state) and they can also form bonds which may be intra- or intermolecular. This approach allows a close mapping between known structural features of proteins and their BNGL representations [28].

For instance, consider a receptor that binds a ligand molecule and has an ion channel that may be in an open or closed state. The BNGL representation for this simple receptor would be: $\operatorname{Rec}\left(\mathrm{lig}, \mathrm{ch}^{\sim}\right.$ open ${ }^{\sim}$ closed). Here, Rec represents the receptor itself and its components are represented in the parentheses, separated by commas. The lig component represents the ligand binding site and ch represents the channel. The states of the channel are indicated by the operator and the possible states are listed; there is no limitation on the number of states. The corresponding representation for ligand that activates the receptor would be much simpler: $\operatorname{Lig}(r e c)$. Since ligands are typically small molecules, they need only minimal internal structure in BNGL-in this case just a binding site for the receptor. An enzyme and its substrate form another pair of molecules often considered in models of signal transduction. In this case, the enzyme representation might be Enz(cat), where the cat component represents the catalytic domain of the enzyme. The corresponding substrate representation might be $\operatorname{Sub}\left(\operatorname{phos}^{\sim} \mathrm{U}^{\sim} \mathrm{P}\right)$, where the act component represents 
a phosphorylation site that may be in unphosphorylated (U) or phosphorylated (P) states.

It is important to note that the molecule types presented above define the basic types of molecules that can exist in the system; they are essentially templates. During simulation, these types are instantiated as chemical species in which any components with several possible states take on a single state. Chemical species may also consist of complexes of molecules connected by bonds between their internal components. While BioNetGen does not enforce a limit on the number of molecules that can be present in a complex, there are practical limitations in terms of the time required for network generation and simulation of models with large complexes. In general, chemical species consisting of ten or fewer molecules are easily handled in BioNetGen $[28]$.

The next step is to define reaction rules that specify how molecules can interact. The key concept here is that the rules are specified in terms of chemical patterns rather than chemical species. Since the patterns in a rule may match a number of species, each rule defines a class of reactions that can occur in the system; during network generation, all possible matches to the rules are converted into explicit reactions with fully specified chemical species. Here, we provide some examples of how rules work using the generic molecule types described above. First, we can represent the binding of the receptor and ligand with the following rule:

$\operatorname{Rec}\left(\right.$ lig, $\mathrm{ch}^{\sim}$ closed $)+\operatorname{Lig}(\mathrm{rec}) \longleftrightarrow->\operatorname{Rec}\left(\mathrm{lig}\right.$ !1, ch $\left.{ }^{\sim} \mathrm{closed}\right) \cdot \operatorname{Lig}(\mathrm{rec}$ !1) kf, kb This rule displays the basic features of BNGL rules. The left-hand side specifies the reactant patterns, followed by an arrow (bidirectional in this case), followed by the product pattern, and finally the rate constants for the forward and backward 
reactions. Note that we have specified the ch component of Rec is in the closed state, which means that the pattern will only match Rec species with closed ion channels. The internal components of each molecule that are involved in the binding are specified on the left-hand side, and the actual bond between them is on the righthand side. Bonds consist of the ! operator followed by a label, which is typically an integer (although it may be any text); this example uses 1 as the bond label. Additionally, the complex is indicated by the operator. We can then represent the activation of the receptor with the following rule:

$\operatorname{Rec}\left(\operatorname{lig} ! 1, \mathrm{ch}^{\sim} \mathrm{closed}\right) . \operatorname{Lig}(\mathrm{rec} ! 1) \longleftrightarrow->\operatorname{Rec}\left(\operatorname{lig} ! 1, \mathrm{ch}^{\sim}\right.$ open).Lig(rec!1) kf, kb This rule specifies that the receptor channel should open when when the ligand is bound. Again, we have used a bidirectional rule, but in this case the rate constant for the backward reaction would likely be very low. Additional rules would then specify the dissociation of the ligand, and subsequent deactivation of the receptor. These rules could be represented as follows:

$\operatorname{Rec}\left(\mathrm{lig} ! 1, \mathrm{ch}^{\sim}\right.$ open). $\operatorname{Lig}(\mathrm{rec} ! 1) \rightarrow \operatorname{Rec}\left(\mathrm{lig}, \mathrm{ch}^{\sim}\right.$ open $)+\operatorname{Lig}(\mathrm{rec}) \mathrm{kf}$ $\operatorname{Rec}\left(\mathrm{lig}, \mathrm{ch}^{\sim}\right.$ open) $\rightarrow \operatorname{Rec}\left(\mathrm{lig}, \mathrm{ch}^{\sim} \mathrm{closed}\right) \mathrm{kf}$

Note that we have used unidirectional rules here because these steps are usually not reversible. However, in some cases, we may also want to allow the ligand to bind when the receptor is in the open state. For this, we could simply omit the ch component from the original binding rule as follows:

\section{$\operatorname{Rec}($ lig) $+\operatorname{Lig}(r e c)<->\operatorname{Rec}(\operatorname{lig}$ !1). $\operatorname{Lig}(r e c ! 1) k f, k b$}

In this case, the rule would match to any Rec species that is not already bound to a ligand, but the state of the ion channel would be irrelevant.

The above rules present the basic concepts of rules in BNGL. We provide one more 
example that demonstrates how enzymatic reactions were represented in the models presented later in this thesis. Consider the following rules:

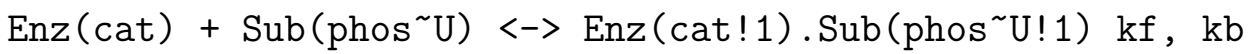

Enz (cat!1).Sub (phos $\sim$ U!1 $\rightarrow \operatorname{Enz}($ cat $)+\operatorname{Sub}\left(\right.$ phos $\left.^{\sim} P\right)$ kcat

The first rule represents the association (and dissociation) of the enzyme and substrate, in the catalytic domain of the enzyme binds the phosphorylation site on the substrate. The second rule represents the catalytic step in which the phosphorylation state of the substrate is changed, and the enzyme and substrate dissociate. These rules represent the elementary reaction steps of enzymatic reactions: association, dissociation, and catalysis. This topic is discussed in more detail in Section 3.3. 


\section{Chapter 3}

\section{Related Work}

This chapter reviews the state of the art in the two computational modelling areas most relevant to this thesis: the induction of synaptic plasticity and scaffold-mediated signal transduction. Computational neuroscience is a broad and diverse field, with a large number of studies that model synaptic plasticity from a variety of different perspectives, levels of detail, and specific synaptic systems (such as cortical, hippocampal, striatal, and cerebellar); indeed, a 2010 paper reviewed and classified some 117 models published between 1985 and 2009, the authors of which repeatedly apologize for excluding many other models [63]. Despite the fact that several prominent researchers in the field have drawn attention to the importance of scaffold proteins at the synapse $[9,13,53,71]$, no known computational modelling work has addressed the effects of scaffolding on synaptic signal transduction. Part of the reason for this is surely due to the sheer number of scaffolds in the postsynaptic density [42] and our current lack of detailed kinetic data for most of their interactions [89]. Another important factor is that modelling methods capable of handling the combinatorial complexity associated with scaffold-mediated signalling [34] have only recently become available [35, 49, 82]. 
The goal of this thesis is to take a first modest step toward understanding the effects of scaffold proteins on synaptic plasticity. Given the lack of any previous work on this topic, it makes sense to try to combine the best understood aspects of the respective fields, ideally in way that will contribute to both. As noted in the Introduction, computational studies of scaffold-mediated signalling are also at an early stage, but several models have characterized the core properties and effects associated with these systems. All of the models considered here (and, indeed, all known modelling studies on scaffolds) focus on the mitogen-activated protein kinase (MAPK) cascade, a canonical signalling pathway that is present in virtually all eukaryotic cells from yeast to humans [37]. In fact, all known forms of the MAPK cascade are known to involve a scaffold [51, 95], so it is quite natural that this system has attracted most (if not all) of the attention of modellers. The MAPK cascade is not only present at the synapse, but it interacts with two other enzymes (PKC and $\mathrm{PLA}_{2}$ ) to form a positive feedback loop that is known to be critical for the induction of synaptic plasticity [12, 91] (see Sections 2.2.1 and 2.2.2 for background material).

The majority of the chapter focuses on models of synaptic plasticity, with primary emphasis on the role of feedback loops in the induction of long-term plasticity; we discuss the role of individual and coupled feedback loops in Sections 3.1.1 and 3.1.2, respectively. Next, we review the literature on scaffold-mediated signalling in the MAPK cascade, focusing on the concentration effects (Section 3.2.1), regulatory roles (Section 3.2.2), and threshold effects (Section 3.2.3); we provide a summary of the limitations of this work in Section 3.2.4. In Section 3.3, we discuss some of the recent methodological trends in modelling enzymatic reactions, and highlight the benefits of modelling elementary reaction steps. Finally, we conclude with a summary of the 
related work in Section 3.4.

\subsection{The Role of Feedback Loops in Synaptic Plas- ticity}

Synaptic plasticity is the activity-dependent modification of the strength of connections between neurons. Research over the last decade demonstrates that these changes can occur very rapidly (on time scales of seconds to minutes) and that they occur continuously throughout the life of an organism. Thus, it is absolutely crucial for synapses to be able to switch quickly and easily between different forms of plasticity (primarily LTP and LTD). This requirement for switching behaviour has drawn researchers to discover and explore the properties of positive feedback loops in synaptic signal transduction networks. Under appropriate conditions, positive feedback is well-known to give rise to bistable systems with two stable steady states (one of low activity and the other of high activity) and a sharp threshold for transitioning between them.

\subsubsection{Single Feedback Loops}

This section discusses the effects of individual positive feedback loops on synaptic signalling. We begin by describing research that investigates how the MAPK-PLA $2_{2}^{-}$

PKC loop (MPPL) generates bistable, switch-like synaptic responses. We also discuss the effects of stochastic noise on synaptic signalling and the possible mitigating role of positive feedback. 


\section{Bistable Behaviour of the MAPK-PLA2-PKC Positive Feedback Loop}

Bhalla and colleagues pioneered the study of the MPPL and established many of its core properties in a series of papers during the late 1990s and early 2000s. As mentioned above, the loop was proposed by Bhalla and Iyengar in 1999 [10]. While this study features synaptic signalling molecules and parameters derived from neuronal sources, its focus is less on the nature of synaptic plasticity than on the importance of emergent properties in signal transduction networks - one such emergent property being the formation and activation of feedback loops. They began by establishing that the system is bistable. There are several ways to do this, but the method used here is to construct one dose-response curve for the steady-state concentration of activated ERK at different initial concentrations of active PKC and a second curve for the opposite case of activated PKC at different concentrations of ERK. When these two curves are plotted on the same axes, the number of intersection points indicates whether the system is bistable or monostable. If there is only one intersection point, then the system is monostable, with the single point representing the basal activity state. If, on the other hand, there are three intersections, then the system is bistable: the lowermost point represents the basal state of low activity, the uppermost point represents the high activity state, with the middle point as the threshold for switching between states [10]. (Other numbers of intersection points are theoretically possible but biologically implausible, and were not observed in this study.)

Bhalla and Iyengar further characterized the MPPL by simulating its deactivation by phosphatases and its robustness to variations in parameters. For deactivation, they focused on the dephosphorylation of ERK by MKP because the latter is known to be

dynamically regulated at the synapse. By stimulating the model past threshold and 
measuring the resulting output of active ERK at different concentrations of MKP, they showed that low levels of MKP had only a transient affect on the active state of the loop, but high levels of MKP could efficiently deactivate the loop and bring the network back to basal state [10]. Finally, Bhalla and Iyengar tested the robustness of the MPPL by randomly varying the values of key reaction rates. They found that the bistable behaviour persists despite large changes in most parameters (over a +/$100 \%$ range of default values), indicating a highly robust system.

In two follow-up studies $[11,12]$, Bhalla and colleagues studied the MPPL more systematically and in greater detail. The core network and methods are essentially identical to the earlier study, but these new models include a negative feedback loop in which ERK activates MKP, which in turn deactivates ERK. The main reason for the inclusion of this negative loop was the suggestion that interlinked positive and negative feedback loops can generate oscillatory responses [43]. These studies focus primarily on the effects of negative regulators (the phosphatases PP2A and MKP) on the bistability of the system. First, Bhalla and Iyengar [11] showed that bistability is retained over the entire physiological range of PP2A concentrations. This suggests that PP2A is not a significant regulator of system dynamics; the action of PP2A only reduces the steady-state output of the network, but does not affect its bistable character. MKP, on the other hand, was found to have a significant effect on bistability. At sufficient concentrations, MKP can convert the response from bistable to monostable; importantly, the system produces graded, proportional responses to inputs in the monostable region [12]. These results suggest that MKP can not only modify steady-state output (as expected) but can also change the system dynamics from proportional to switch-like responses under appropriate conditions. Bhalla and 
colleagues hypothesize that this allows for flexibility and dynamic control of network responses. Finally, it is important to note that these two studies $[11,12]$ also examined whether negative feedback loops in this network could generate oscillating responses. When using physiologically plausible concentrations and reaction rates, they did not observe any oscillatory behaviour, although such behaviour could be generated by pushing the parameters beyond physiological ranges [11, 12].

\section{Positive feedback and the Effects of Stochastic Noise}

As in the field of biochemical modelling more generally, the majority of synaptic plasticity models are deterministic models consisting of systems of differential equations. Comparably few studies have used stochastic methods - but, when these methods are used, they often produce results that diverge significantly from the deterministic results. Deterministic methods are still predominant largely because they are much more efficient and allow more extensive exploration of parameter space. However, stochastic effects are especially important for synaptic plasticity because the low volumes and copy numbers of key molecules are precisely the conditions under which stochasticity is expected to be important. Several studies have shown that models which are bistable under deterministic conditions may not be under stochastic conditions. Positive feedback loops are predicted to have a mitigating effect here, potentially restoring bistability. In this section, we briefly describe the overall effects of stochastic noise and the role of positive feedback.

As discussed above, Bhalla and colleagues had shown strong and robust thresholding and bistability in synaptic signal transduction (other work had shown interesting

temporal tuning properties that are less relevant to here $[4,5])$. Importantly, all 
of these studies employ strictly deterministic methods. In 2004, Bhalla published a pair of papers $[7,8]$ in which he compared the results of deterministic and stochastic simulations of a set of similar models. He found that stochastic noise severely diminished all three properties (thresholding, bistability, and temporal tuning) at typical synaptic volumes. Specifically, his results showed that the system no longer possessed a fixed threshold for activation and that the output would spontaneously switch from between the low and high activity states so that the response was no longer all-or-none. In other words, activation of the network was probabilistic and the activity state was unstable $[7,8]$. Antunes and De Schutter [3] recently studied a more detailed model that largely mirrored these results. (It is important to note, however, that there are considerable differences between these models, both in terms of their construction and responses, which are discussed below and in more detail in section 3.3.)

Smolen et al. [87] studied a simpler model of the standard MAPK cascade and focused on the interaction between stochasticity and positive feedback. They first showed that a version this of model with no feedback loops was bistable under deterministic conditions. Then they performed the same simulations again, this time using stochastic methods and the low molecule counts found at the synapse, and found that bistability was lost, much as in Bhalla's work. Finally, they added a positive feedback loop by allowing ERK at the end of the cascade activate Raf at the beginning (a reaction known to occur in some cell types) and found that this simple loop not only restored the bistable response, but also extended the bistable region to a larger range of concentrations of the components [87]. These results suggest that positive feedback loops are capable of stabilizing the responses of stochastic models and po- 
tentially bringing them into closer alignment with deterministic models. Smolen and colleagues chose the ERK-Raf feedback because it is fairly simple and had not previously been examined in a synaptic context. It is interesting to note, however, that the Bhalla model [8] and the Antunes and De Schutter model [3] both include the MAPK-PLA2-PKC positive feedback loop, but with different effects on the stability of responses: the former being unstable with randomly flipping states and the latter probabilistically activated but with stable all-or-none responses. Thus, while only a few studies have been conducted, the evidence so far suggests that single feedback loops are of limited use in stabilizing stochastic responses.

\subsubsection{Coupled Feedback Loops}

There are two major proposals for the role of coupled feedback loops in synaptic signalling: enhanced robustness to stochastic noise and regulation of bidirectional plasticity. In the former case, Ogasawara and colleagues [71, 73] have explored the properties of systems with several interlinked feedback loops that operate at different time scales. In the latter case, Kikuchi et al. [44] and Pi and Lisman [78] have studied so-called tristable synaptic systems composed of coupled kinase and phosphatase loops which control the induction of LTP and LTD. In this section, we discuss the key results of these studies.

\section{Cascades of Excitable Dynamics and Stochastic Noise}

Ogasawara and colleagues have published several papers on the role of interlinked positive feedback loops in providing robustness to stochastic noise in the induction

of cerebellar long-term depression $[71,73,72,74]$. Known as the cascade of excitable 
dynamics (CED), their proposal is fairly straightforward: three positive feedback loops with fast, medium, and slow kinetics that sequentially feed into each other can provide synaptic systems with the flexibility to acquire new information and the stability to store it over the long term [72].

They have also identified feedback loops that could fill these roles. The first is well-known in many areas of cell biology and involves calcium-induced calcium release (CICR), which occurs on time scales of a few seconds. Here, synaptic stimulation initially triggers influx of $\mathrm{Ca}^{2+}$ through ligand- or voltage-gated ion channels, thereby increasing intracellular $\mathrm{Ca}^{2+}$ concentration. Among others, the increased $\mathrm{Ca}^{2+}$ concentration activates PLC $\beta$, which in turn produces $\mathrm{IP}_{3}$ and DAG from PIP2 (a common phospholipid component of the plasma membrane). $\mathrm{IP}_{3}$ then binds to receptors on the endoplasmic reticulum and releases more $\mathrm{Ca}^{2+}$ from internal stores. This further increase in $\mathrm{Ca}^{2+}$ again activates $\operatorname{PLC} \beta$ and closes the loop [26, 73]. The second loop is the MAPK-PLA2-PKC loop (MPPL) that we have already discussed at length, which connects to the CICR loop at several points. $\mathrm{Ca}^{2+}$ activates both PLA2 and PKC, and PKC is additionally activated by the DAG produced by PLC $\beta$. The MPPL functions at time scales of tens of minutes [54, 71, 92]. The final loop is newer and less well characterized, but it involves the atypical PKC isozyme known as $\operatorname{PKM} \zeta$, which is a persistently active form of the enzyme (i.e., it contains the catalytic domain of $\mathrm{PKC} \zeta$ but lacks the regulatory domain). The core mechanism of this loop is that $\mathrm{PKM} \zeta$ is known to induce its own expression, and thus we can call this the $\mathrm{PKM} \zeta$ induced $\mathrm{PKM} \zeta$ expression (PIPE) loop. $\mathrm{PKM} \zeta$ expression is initially induced by ERK, providing a connection to the MPPL. The time scales of PIPE are much longer than the other loops and can last from days to months $[73,74]$. 


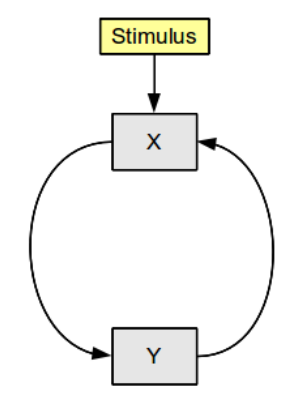

Figure 3.1: Single feedback loop model used by Ogasawara and Kawato in [73].

In order to examine this proposal, Ogasawara and Kawato [73] constructed simplified models of positive feedback loops and conducted computational experiments under deterministic and stochastic conditions. The goal was to determine whether and under what conditions such models could overcome stochasticity while remaining responsive to important inputs. The models used in this work include a single loop model and a double, interlinked loop model (see figures 3.1 and 3.2. These models are highly idealized in that they abstract away from the biochemical details to focus on the time constants of the interactions. For each model, Ogasawara and Kawato performed deterministic and stochastic simulations with different combinations of slow and fast kinetics. The stimuli for these simulations were brief, one second pulses of an input signal [73].

For the deterministic single loop with fast kinetics $(\tau=1 s)$, a weak stimulus produces only a transient response followed by return to baseline, and a strong stimulus fully activates the loop; at equilibrium, the loop is either fully inactive or fully active - a bistable switch. With slow kinetics $(\tau=10 \mathrm{~s})$, the single loop model is essentially insensitive to stimuli because they shorter than the time constant for activation of the loop by a factor of ten, so that stimuli end before the loop can become 


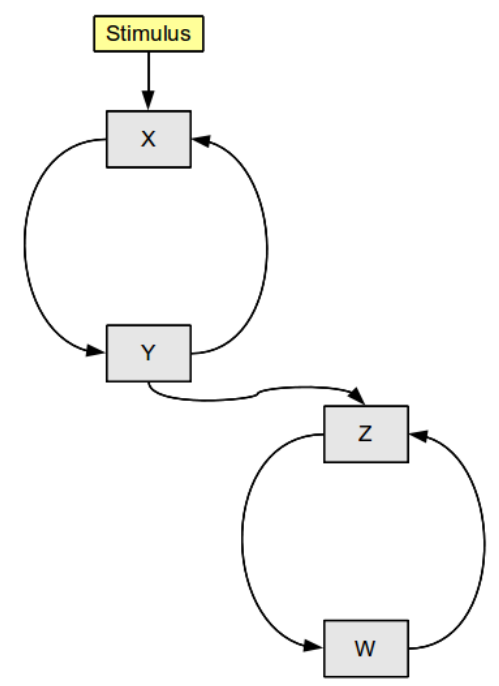

Figure 3.2: Coupled feedback loop model used by Ogasawara and Kawato in [73].

active. Under stochastic conditions (small volume and low numbers of molecules), the same parameters yield very different results. Here, there are frequent spontaneous transitions between the active and inactive states, much as Bhalla had found. Ogasawara and Kawato found that the length of time that the loop would remain active was proportional to $\tau$; for small $\tau(1 \mathrm{~s})$ and a strong stimulus, the loop is transiently active, but larger $\tau(10 \mathrm{~s})$ and a very strong stimulus can activate the loop and keep it active for relatively long periods of time. This indicates that there is a tradeoff between stability and responsiveness under stochastic conditions.

In the case of two coupled loops, Ogasawara and Kawato connected the fast and slow loops used above, such that the stimulus activates the fast loop and the output of the fast loop activates the slow loop (see figure 3.2); the time constants of the loops are also based on those of the single loops - that is, $\tau=1 s$ for the fast loop and $\tau=10 \mathrm{~s}$ for the slow loop. In deterministic simulations, the fast loop consisting of $\mathrm{X}$ 
and $\mathrm{Y}$ behaves much like the single loop with fast kinetics: a bistable response with strong thresholding. The slow loop could not be activated in isolation but gradually transitions to the active state as the fast loop activates, showing a marked increase in sensitivity that is likely due to the amplification of the signal by the fast loop. Under stochastic conditions, strong stimuli transiently activate the fast loop, often long enough for the signal to propagate to the slow loop. As in the single loop case, the slow loop is difficult to activate but, once activated, it is likely to remain active for a long time. Here again, the fast loop amplifies the signal enough to activate the slow loop. The end result is that coupled fast and slow loops can be more sensitive to incoming stimuli than a single slow loop, while also being more stable than a single fast loop.

\section{Tristable Systems for Bidirectional Synaptic Plasticity}

Research on synaptic plasticity has uncovered two basic classes of plasticity: longterm potentiation (LTP), which strengthens a given synapse, and long-term depression (LTD), which weakens a given synapse. Initially, researchers speculated that LTD was simply the reverse of LTP, but it later became clear that they involve different pathways. Furthermore, the evidence increasingly suggests that both of these pathways are bistable. This gives rise to the idea that there are three basic states

of the synapse: unpotentiated (or baseline), potentiated, and depressed [78]. Several computational studies have examined this 'tristable' system to understand bidirectional synaptic plasticity.

Pi and Lisman [78] studied a simplified model of the induction LTP and LTD in the hippocampus that focuses on the opposing roles of the kinase CaMKII and the 


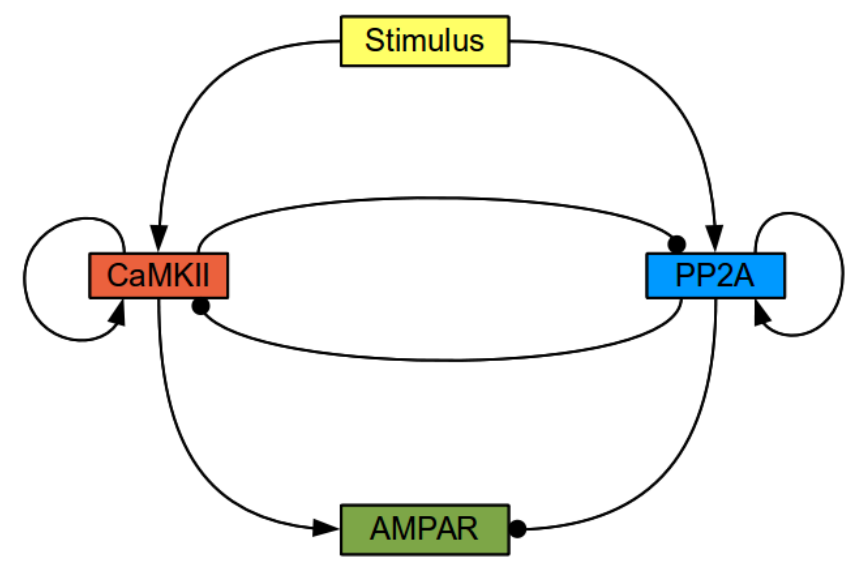

Figure 3.3: Tristable synaptic system. CaMKII and PP2A each have autoactivation loops and inhibitory connections between them. Adapted from [78].

phosphatase PP2A. The model ignores many of the details and reaction steps, but preserves the essential features. It is essential to note at the outset that, while it is sometimes assumed that phosphorylation means activation and dephosphorylation means deactivation, this is not always the case: for PP2A, the phosphorylated form is the inactive form. Figure 3.3 shows the basic configuration. The stimulus $\left(\mathrm{Ca}^{2+}\right.$ in this case) activates both CaMKII and PP2A. Once active, CaMKII can phosphorylate and activate other CaMKII, forming a positive feedback loop. CaMKII also phosphorylates and deactivates PP2A. Active PP2A can dephosphorylate and activate other PP2A, forming a second positive feedback loop. PP2A also dephosphorylates and deactivates CaMKII (the phosphorylated form of CaMKII being the active form). Finally, these two loops affect plasticity by acting on AMPA receptors, with CaMKII enhancing their conductance and PP2A diminishing it.

This model is similar to the Ogasawara-Kawato model discussed in the previous section in that both involve interlinked positive feedback loops. The key difference is 
that the Pi-Lisman model features bidirectional inhibitory connections between the loops where the Ogasawara-Kawato model has a unidirectional stimulatory connection from the fast loop to the slow loop. The inhibitory connections allow the loops to deactivate each other and switch the system among multiple states. The two models also have different motivations: Ogasawara and Kawato wanted to understand how synaptic systems can overcome stochasticity, while Pi and Lisman were interested in how bidirectional plasticity works. In this respect, it is important to note the Pi-Lisman model is deterministic and has not been tested using stochastic methods.

Using their model, Pi and Lisman showed that a synaptic system can have three stable states necessary to support bidirectional plasticity. First, simply running the system to equilibrium without any $\mathrm{Ca}^{2+}$ stimuli yields the basal, unpotentiated state. Then they applied brief $\mathrm{Ca}^{2+}$ pulses at various concentrations and found sharp thresholds for activation of the PP2A and CaMKII autocatalytic loops. The basal state results from pulses between 0.1 and $0.5 \mu \mathrm{M}$. Pulses between 0.6 and $2.5 \mu \mathrm{M}$ activate the PP2A loop and the system settled into the depressed state. There was an unstable region between 2.6 and $2.9 \mu \mathrm{M}$ of $\mathrm{Ca}^{2+}$ in which neither LTD or LTP is induced. Finally, pulses of $3.0 \mu \mathrm{M}$ or greater activate the CaMKII loop and the potentiated state. Each of these three states are persistently activated by brief $(2 s)$ stimuli at the appropriate concentrations. Importantly, the potentiated and depressed states can be returned to the basal state by applying the opposing stimulus; if the system is in the potentiated state, then a $\mathrm{Ca}^{2+}$ pulse in the LTD range (0.6 to $\left.2.5 \mu \mathrm{M}\right)$ will deactivate the CaMKII loop and return the system to the basal state (and vice versa) [78]. Thus, the system is truly tristable and appropriate stimuli are capable of switching among any of the states. 


\subsection{The Role of Scaffold Proteins in MAPK Sig- nalling}

In this section we review some of the recent work on scaffold-mediated signalling. As noted in the introduction to this chapter, much of the research on scaffold proteins focuses on the MAPK cascade. Modelling work in this area is at an early stage, but several key properties have been identified and explored in computational studies, including concentration effects, regulatory roles, and impact on thresholding and bistability. Each of these topics are addressed in the sections below.

\subsubsection{Concentration Effects}

One of the most robust findings in this literature is that there exists a range of scaffold concentrations which optimally support signalling in terms of both the amplitude and duration of output signals $[49,52,57,59,95]$. Generally known as the prozone effect, Levchenko and colleagues first reported this result and showed that increasing the scaffold concentration generates a stronger and more sustained response (as measured by the production of activated ERK) up to a threshold, after which further concentration increase inhibits the response [57]. While experimental work has largely confirmed this prediction, the optimal range in the experimental system is much wider than that calculated for the models $[52,95]$. Later computational work showed that the range is determined not only by the concentration of the scaffold but also by the relative concentrations of the enzymes involved in the cascade. When the concentrations of Raf, MEK, and ERK are assumed to be similar (as is the case in [57]), then the range of optimal scaffold concentrations is narrow; however, when the 
enzyme concentrations are more diverse (as is the case in most experimental measurements), then the optimal scaffold range is wider $[95,96]$. In other words, this optimal scaffold range is determined by the spread of enzyme concentrations.

The explanation for the range of optimal scaffold concentrations is simple and intuitive $[52,95]$. Suppose that we have a system with 2 Raf, 30 MEK, and 20 ERK molecules (these values are chosen because they are proportional to typical synaptic values for the enzymes). The least abundant component (Raf in this case) determines the maximal number of full complexes and there must be at least this many scaffold molecules in order to form the maximum number of full complexes; thus, the least abundant component determines the lower bound of the scaffold concentration range (2 molecules in this case). For any number of scaffolds between 2 and 20 (the least abundant and second least abundant components in this example), each scaffold can bind a MEK and ERK molecule (as these are in excess), leaving the Raf molecules to bind any two scaffolds in order to produce the maximum number of full complexes. As the number of scaffolds exceeds 20, the proportion of scaffolds that have only MEK bound increases and the probability that the Raf molecules will bind scaffolds with both MEK and ERK to produce full complexes decreases. Thus, the upper bound of the scaffold range is determined by the second least abundant component (ERK at 20 molecules in this case). This basic picture is complicated by the fact that the binding constants of the individual enzymes can also affect the placement and width of the optimal scaffold range, but it nonetheless provides a useful starting point for understanding the effects of scaffold concentration on signalling [95]. 


\subsubsection{Regulatory Roles}

Scaffold proteins can play a variety of roles in the regulation of signalling outputs. Locasale and colleagues $[60,59]$, in particular, have explored the effects of scaffolds on the amplitude, timing, and duration of MAPK signalling. First, Locasale et al. [60] examined the effect of scaffolds on the amplitude of signalling outputs. This work showed that scaffolds can, in some cases, amplify signals that would be attenuated in their absence and, conversely, that under other conditions scaffolds can suppress signals that would otherwise be amplified. To simulate these conditions, they modified the amount of phosphatase activity and varied the affinity with which scaffolds and kinases bind to each other. Under high phosphatase activity, the kinases are suppressed and the cascade is difficult to activate; more specifically, weak signals are attenuated before they can propagate to the end of the cascade to produce a cellular response. They then added a scaffold that binds all three kinases and found a sharp increase in the concentration of activated kinases and enhanced signal propagation. In further simulations, they explored the opposite case in which phosphatase activity is low and signals quickly propagate to the end of the cascade. Here, signals are strongly amplified because the kinases are largely unopposed by phosphatase activity. Under these conditions, the addition of the scaffold actually attenuates the signalling output and slows propagation; there is still some amplification, but the effect is dampened compared to the case without the scaffold. In both of these cases, it is important to note that the binding affinity between the scaffold and the kinases determines whether signalling will occur primarily in the cytoplasm or on the scaffold, and hence whether the overall amplitude of the output will be amplified or attenuated [60].

To account for this dual amplify-attenuate role of scaffold proteins, Locasale et al. 
propose a simple underlying mechanism. It is well-known that scaffold-mediated signalling involves certain stoichiometric constraints: an active kinase in the cytoplasm can phosphorylate any substrate in its vicinity, but a kinase bound to a scaffold is restricted to acting on substrates also bound to the same scaffold $[52,95]$. The number of substrates that a cytoplasmic kinase can activate, $N_{c y t}$, depends on its diffusion rate and the time required for that kinase to be deactivated by a phosphatase. Under the standard assumption that phosphatases only act on kinases in the cytoplasm, the corresponding number for a scaffold-bound kinase, $N_{\text {scaff }}$, depends only on the number of scaffold-bound substrates. Thus, when phosphatase activity is low, $N_{c y t}>N_{\text {scaff }}$ and the kinase can activate more of its substrates in the cytoplasm than on the scaffold. On the other hand, when phosphatase activity is high, $N_{c y t}<N_{\text {scaff }}$ and the scaffold amplifies signalling. Again, the binding affinity is critical for directing signalling to the cytoplasm or the scaffold and determining whether amplification or attenuation will occur [60].

In a follow-up study, Locasale and Chakraborty [59] investigated how scaffolds affect the duration of MAPK signalling, this time focusing more on the role of scaffold concentration. To begin with, they observed the same type of optimal range for scaffold concentration discussed in the previous section. However, Locasale and Chakraborty went farther and explored how scaffold concentration affects the time scales of signal propagation. At both low and high scaffold concentrations, they found that signalling is dominated by a single time scale. But near the optimum concentration, signals could propagate over multiple time scales, yielding a richer and more diverse signalling profile. The explanation for these observations is fairly straightforward. When scaffold concentration is low, most signalling occurs in the cytoplasm 
and its time scale is determined by the association rates of the kinases in solution. Conversely, when scaffold concentration is high, most kinases are bound to incomplete complexes and the time scale is determined by the the time it takes for complete complexes to form. When the scaffold concentration is between these extremes in the optimal range, however, kinases can exist in all of these states (cytoplasmic association, incomplete complex, and complete complex) with transitions among them, producing a mixture of time scales. In other words, when kinases are activated primarily in one of these states, that state will determine a dominant timescale, but when there are multiple activation pathways, multiple time scales will emerge [59].

Taken together, these two studies $[60,59]$ demonstrate that scaffold proteins can dramatically alter the signalling properties of MAPK cascades by selectively altering the amplitude and timing of output responses. The authors speculate that cells employ combinations of these strategies to produce complex signalling profiles and that allosteric modulation and cooperative binding would allow for dynamic regulation and adaptation to specific cellular contexts $[60,59]$. What is clear is that scaffolds are important regulators of signalling dynamics that interact with different components of the network in complex ways.

\subsubsection{Thresholding and Bistability}

Another important aspect of scaffold function in MAPK signalling is how scaffolds affect the bistability of the response. As discussed in Section 2.2.2, ultrasensitive and/or bistable responses are a hallmark of the MAPK cascade, which may be generated through a variety of mechanisms. The most commonly cited mechanism is that MEK and ERK require two phosphorylation events separated by full dissocia- 
tion of enzyme and substrate (that is, multistep distributive activation). Relaxing the requirement for dissociation between phosphorylation yields the so-called processive reaction mechanism, which, in the absence of scaffolds, has been shown to convert the bistable all-or-none response into a graded proportional response (see, e.g., [15, 30]). Given this, many researchers have wondered whether the addition of a scaffold would allow both of the necessary phosphorylation events to occur during a single encounter with the scaffold, thereby producing an essentially processive reaction mechanism. If so, the key question then is whether this processive reaction on the scaffold will abolish (or at least diminish) the bistability of the response as in the cytoplasmic case.

Levchenko et al. [57] were the first to address this question and compared three models: two scaffold models, one with full dissociation between phosphorylation events (the distributive case) and one without (the processive case), and a cytoplasmic model, in which catalysis was distributive. To assess bistability, they constructed dose-response curves for each model over a range of scaffold concentrations; these curves are typically sigmoidal and examination of the steepness of the inflection provides a general indication of bistability, since this suggests a sharp threshold. The results showed that, as the scaffold concentration increased, the dose-response curves for the processive model became more graded, indicating a softening of the threshold. In contrast, the distributive curves displayed sharp thresholds that were similar to the cytoplasmic curves, but the magnitude of the outputs was much lower. While initially unexpected, this inhibition effect in the distributive model makes good sense. A fully distributive reaction mechanism involving a scaffold requires that an active kinase and its substrate bind to the same scaffold, whereupon the kinase phosphorylates the substrate and both dissociate from the scaffold. Then each must again bind 
to another scaffold to undergo the final phosphorylation. In the meantime, the kinase and the substrate are subject to dephosphorylation by a phosphatase in the cytoplasm. Thus, the process involves four diffusion-limited association reactions (twice as many as the cytoplasmic or processive scaffold cases) and ample opportunity for deactivation in the cytoplasm [57]. Overall, these results support the hypothesis that processive scaffold reactions tend to weaken activation thresholds, producing more graded responses than their distributive cytoplasmic counterparts.

While Levchenko et al. study only studied the effects of varying the concentration of scaffolds in their models, other researchers have shown that the binding affinities between the scaffold and kinases can also convert a bistable/ultrasensitive response into a graded one and vice versa. Thalhauser and Komarova [93] explored wide ranges of parameters and showed that certain parameter regimes yield graded or bistable responses, in models both with and without scaffolds. In general, their results demonstrate that weak to moderate binding affinities tend to produce bistable responses, while graded responses result from very tight binding [93]. Chan and colleagues [20] observed similar effects of binding affinity. Moreover, Thalhauser and Komarova also found that differences in the upstream activation steps of the first kinase in the cascade could also affect whether the response was bistable or graded [93].

\subsubsection{Limitations of Scaffold Models}

The scaffold models discussed in the previous sections provide evidence for some important effects of scaffold proteins in MAPK signalling. However, there is disagreement regarding the effects of scaffolds on the activation threshold of the MAPK 
cascade and the underlying mechanisms of those effects. The possible sources of these effects include differences in: reaction mechanisms (distributive versus processive), scaffold binding affinities, and upstream activation steps. It is certainly possible that cells use these different mechanisms to tailor the effects scaffolds have in specific contexts. However, a possible methodological reason for the conflicting reports that has not been identified previously is that these models treat the rates of cytoplasmic and scaffold reactions differently - whether by directly assuming a higher rate on the scaffold, using different reaction orders or mechanisms for one than the other, or both. Specifically, Thalhauser and Komarova [93] and Locasale et al. [60, 59] assume different catalytic rates for cytoplasmic versus scaffolded reactions, Levchenko et al. [57] use different reaction mechanisms (Michaelis-Menton kinetics in the cytoplasm versus first-order reactions on the scaffold), and Kocieniewski et al. [49] use both different catalytic rates and different reaction orders. As discussed in the next section, these types of methodological differences can potentially have a significant impact on the results of simulations.

\subsection{Trends in Biological Modelling}

The previous sections in the chapter have discussed a variety of computational modelling studies without much mention of the approaches and methods used, aside from indicating whether the simulations were deterministic or stochastic. These studies span a nearly fifteen year period over which modelling methods have changed substantially. Section 2.4 discusses systems biology modelling methods in detail; in this section, we present some of the broad trends in the literature. These trends are not only important for understanding the current state of the art, but also because they 
motivate the choice of models and methods used in the present research, particularly in light of the case of scaffold models. Here, we focus on the approximations used in modelling enzymatic reactions because significant changes have occurred in this area that are crucial to the present research.

\subsubsection{Approximations for Enzymatic Reactions}

One of the most obvious trends in the literature is that researchers are increasingly moving towards using more detailed representations of reactions. We see this quite clearly for enzymatic reactions, where there has been a transition from the MichaelisMenton approximation (MMA) to using so-called elementary reaction steps (ERS).

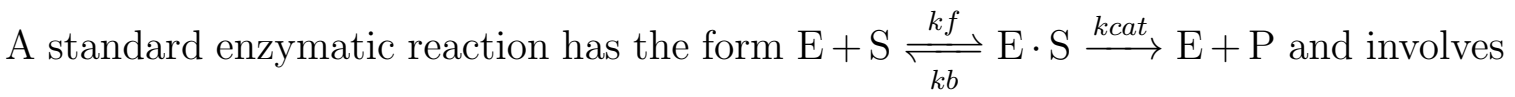
three core steps: i) in the association step, the enzyme (E) and the substrate (S) can bind to form the complex ES; ii) in the dissociation step, the ES complex can unbind to yield $\mathrm{E}$ and $\mathrm{S}$; iii) in the catalytic step, the ES complex can react to produce the enzyme $\mathrm{E}$ and the product $\mathrm{P}$. The MMA combines these three steps into a single reaction that uses parameters derived from the rate constants of the individual steps. This method has the advantage that the intermediate ES complex is never explicitly formed and the number of chemical species in the network is kept to a minimum. The MMA was originally developed for metabolic reactions and its assumptions are typically met in those contexts, and this was assumed to be the case for signalling reactions as well; indeed, most of the papers cited in this chapter that were published before approximately 2010 use Michaelis-Menton kinetics (this includes the work by Bhalla and colleagues [10, 11, 12, 8], Ogasawara and Kawato [73], Pi and Lisman [78], Levchenko and colleagues [57]). 
In 2004, evidence began to surface that the assumptions of the MMA might not be met in signalling contexts [94] and, by 2010, this had become well-established [82]. The alternative to using the MMA is to represent each of the elementary reaction steps (ERS) explicitly in the model. Simulations comparing these two approaches (summarized in [22]) showed not only significant differences in the model responses, but also that models using ERS were in better agreement with experimental data. Since the middle of the last decade, researchers in synaptic plasticity (and in computational biology more generally) have increasingly used ERS. (Work cited in this chapter that uses elementary reaction steps includes Antunes and De Schutter [3], Locasale and colleagues [60, 59], and Smolen et al. [87].) While using ERS may be

more accurate than the MMA, it also requires more parameters (a rate constant for each step), which may not be available; indeed, most experimental studies report still report only the Michaelis-Menton constants. Moreover, using ERS adds intermediate complexes for each enzymatic reaction, which increases the network size and can drastically slow down simulations, particularly for densely connected networks [82]. It is also worth noting that simplified MMA models still have their place and are well-suited to situations in which the researcher wants to understand the essential features of a system without being bogged down with the details.

\subsection{Summary of Related Work}

This chapter has reviewed a sampling of the most relevant work in the fields of synaptic plasticity and scaffold-mediated signalling. We have seen that feedback loops play a critical role in synaptic plasticity. Positive feedback loops naturally generate bistable switches that are ideal for activating (and deactivating) different forms of 
plasticity and they can also have a mitigating effect on stochastic noise, particularly when arranged in a cascade; on the other hand, couple positive feedback loops with inhibitory links between them can give rise to tristable systems that support bidirectional plasticity. We also discussed the regulatory roles of scaffold proteins in the MAPK cascade and saw how scaffolds can have counter-intuitive effects on signalling dynamics. Signalling is optimally supported by a range of scaffold concentrations that depends on the relative abundances of the enzymes involved. Under appropriate conditions, scaffolds can amplify signals that would be attenuated in their absence, and vice versa. Moreover, scaffolds allow a signalling network to maintain multiple time scales of signalling, thereby increasing the flexibility of the network. Finally, scaffolds have a complex relationship to thresholding and bistability in the MAPK cascade. The evidence so far resists a simple classification of this effect, as it has been shown to depend both on the type of reaction mechanism used (distributive vs. processive) and on the specific parameters and reaction mechanisms used. We also identified some potential differences among these effects and briefly described our approach to dealing with them. In the final section, we examined the recent trends in modelling enzymatic reactions and saw that the field is largely moving away from the Michaelis-Menton approximation in favour of explicitly representing the elementary reaction steps.

The fields of synaptic plasticity and scaffold-mediated signalling have done an excellent job of addressing their core concerns independently, and yet these concerns are actually quite relevant to each other. The core concern of synaptic plasticity here is the role of feedback loops, which has not been addressed in any known models of scaffolds. The scaffold literature, for its part, has focused on the regulation of MAPK 
signalling and, even though the MAPK cascade figures prominently in synaptic plasticity, scaffolds have not been considered in synaptic models. Thus, the state of the literature is ripe for cross-fertilization: synaptic researchers need to integrate scaffolds and scaffold researchers need to consider the role of feedback loops. The addition of scaffold proteins to synaptic models and of feedback loops to scaffold models would facilitate this cross-fertilization, and that is what the present research aims to provide. 


\section{Chapter 4}

\section{Materials and Methods}

The previous chapter reviewed important contributions to research on synaptic plasticity and scaffold-mediated signalling, with emphasis on the important regulatory roles of feedback loops and signalling complexes. We saw that research on synaptic plasticity has made considerable progress in understanding how feedback loops shape synaptic responses by acting as switching elements with sharp thresholds, but the field has yet to address the effects of scaffolds or signalling complexes. At the same time, other researchers have established important properties of scaffold proteins, but have disagreed about the effects scaffolds have on the activation threshold of the MAPK cascade, nor have they considered the role of feedback in their models. Therefore, to reiterate our problem statement is, we wish to determine the qualitative effects feedback loops and signalling complexes on the thresholding properties displayed in the induction of synaptic plasticity.

In this chapter, we present the approaches and methods used to address the problem statement. The material is divided into design and implementation. In Section 4.1.1, we describe our application of the systems biology approach. Section 4.1.2 
describes the selection of core synaptic and scaffold models. Section 4.1.3 presents the overall experimental design. We describe the procedures used to implement the selected synaptic and scaffold models in Sections 4.2 .1 and 4.2.2, respectively. Finally, Section 4.2.3 presents the procedure for creating the final models by adding feedback loops and merging the scaffold model into the synaptic model.

\subsection{Design}

The goal of the project was to explore the effects of feedback loops and signalling complexes on the threshold properties displayed in the induction of synaptic plasticity. Therefore, the overall design employs a comparative strategy involving the creation of synaptic models with and without each feature and simulation of those models under conditions relevant to the induction of plasticity. The models are derived from published models but implemented in BioNetGen in order to represent protein interactions at the domain level.

\subsubsection{Application of the Systems Biology Approach}

As defined by Noble and colleagues [50] and outlined in Section 2.4.1, the systems biology approach is a multiscale and middle-out approach. The basic procedure is to define the phenomenon of interest as the middle and then add structure and functionality at higher and lower spatiotemporal scales and/or organizational levels, with the goal of providing just enough detail to support the properties or behaviours under study. The presence or absence of the desired properties is then tested by exploring the parameter space of the model through simulation. This procedure can 
be iterated to enable progressive construction of richly structured biological models. Our aim was to execute one iteration of the procedure to develop a synaptic model with enough structural detail to support the inclusion of scaffold proteins and the formation of signalling complexes.

In our case, the level at which the most data are available is the level of individual proteins; the kinetic parameters used in all of the models reviewed in Chapter 3 represent protein interactions at this level. Since we selected two of these models to use as a base for our work (see Section 4.1.2 below), we define the level of individual proteins as our starting point (the 'middle'). Above the individual proteins are the feedback loops and signalling complexes, followed by the signal transduction network, and finally the dendritic spine itself. We follow the approach taken in most of the work in this area, and do not consider levels above the dendritic spine. The reason for this is that the spine is generally considered to be a relatively isolated subvolume and can thus be considered on its own. Below the individual proteins are the domains and molecular substructures, followed by the amino acids that comprise the domains, and so on. It is important to include protein domains because it is well known that protein interactions to occur at the domain level. Moreover, online databases such as UniProt [25] provide protein sequences and functional annotations for an enormous variety of proteins, making information about domains and phosphorylation sites easily accessible. Considering details below the level of domains or their threedimensional structures is beyond the scope of this thesis. Thus, our main additions to previous models are the domains that mediate protein interactions and scaffold proteins that mediate the formation of signalling complexes. 


\subsubsection{Model Selection}

The first task is to select appropriate models, ideally one each from the synaptic and scaffold literatures that can be merged to produce template models with both feedback loops and signalling complexes. The primary requirements for the models were that they be recent (post-2010), provide enough information for replication in another framework, and use elementary reaction steps rather than Michaelis-Menton kinetics (or other approximations) for enzymatic reactions. The scaffold literature is smaller and thus has more constraints. Indeed, as noted elsewhere, the vast majority of modelling studies focus on the scaffolds of the MAPK cascades - as do all of the models discussed in Chapter 3. Of these, only Levchenko et al. [57] and Kocieniewski et al. [49] provide the full reaction schemes and parameter sets required for replication. The Kocieniewski paper is more recent (published in 2012), provides better reaction information, and also uses BioNetGen for modelling and simulation. These factors make the Kocieniewski model a good choice for the scaffold model.

The selection of a synaptic model is more difficult simply because there are far more synaptic models. Several models were implemented (including [8] and [87]) before settling on the Antunes and De Schutter model of cerebellar LTD, published in 2012 [3]. This model is ideal because it focuses specifically on the MAPK-PLA $A_{2}-\mathrm{PKC}$ feedback loop, incorporates thoroughly researched parameters, and uses elementary reaction steps throughout. Additionally, the full model is available online (written for the STEPS simulator, developed in De Schutter's lab) along with documentation of all reactions and parameters. Thus, the two selected core models are the scaffold model of Kocieniewski et al. and the synaptic model of Antunes and De Schutter. Details concerning how these models were developed and merged are provided in 
Section 4.2.

\subsubsection{Experimental Design}

In order to assess the effects feedback loops and signalling complexes, we constructed models with and without each feature. In terms of feedback loops, we added one positive and one negative loop to our core models, which resulted in three types of feedback models: no added feedback, added positive loop, and added negative loop (see Figures 4.4 and 4.5). In terms of signalling complexes, the core scaffold model focuses on KSR (or Kinase Suppressor of Ras), which is the primary MAPK scaffold in mammals. To study the effects of the scaffold, we needed models with and without scaffolds. Crossing these basic types produced six core models with different combinations of feedback and scaffolding.

To understand the dynamics of these models, we conducted a variety of simulations under different conditions. The choice of which parameters to modify to create these conditions is far from trivial, particularly when each elementary reaction step is represented explicitly with its own rate constant (the full model incorporates nearly 250 rate constants and initial concentrations to consider). Our choices were motivated by simplicity and structural accuracy, with the aim of selecting the smallest number of parameters to control and choosing them in order to affect the most appropriate reaction step. From previous research, we know that the magnitude of the calcium stimulus and the level of phosphatase activity are important regulators of the system, which are often used to set the cellular context for signalling. We thus consider them to be primary regulators and control them by varying their concentrations; by controlling the concentration of calcium stimuli and of phosphatases, we can simulate a 
wide range of cellular conditions without modifying the details of any reactions.

Of course, the most important manipulations involve the strength of feedback loops and the binding affinity for the formation of signalling complexes. Here, we take the simplest approach and control a single rate constant for each case. In each feedback loop, the critical reaction that forms the loop is catalyzed by ERK. To control the strength of the connection, we varied the rate constant for the catalytic step in each reaction. For the scaffold reactions, we controlled only the affinity of the enzymes for the scaffold and assumed that the rate constants for enzymatic reactions on the scaffold is that same as in the cytoplasm (see Section 4.2.2 for more details).

The binding affinity of a reaction is defined as $K_{d}=\frac{k_{b}}{k_{f}}$, where $k_{b}$ is the dissociation rate and $k_{f}$ is the association rate. The binding affinity determines how tightly two molecules bind together with lower values representing tighter binding. To control binding affinity, we varied the dissociation rate $\left(k_{b}\right)$.

Thus, overall we have four parameters of interest: the concentration of $\mathrm{Ca}^{2+}$ stimuli, initial concentration of phosphatases (all varied together for simplicity), the rate constant for the catalytic step of feedback reactions, and the rate constant for the dissociation of enzymes from the scaffold. In our simulations, we varied these parameters individually and in pairs over biologically plausible ranges.

\subsection{Implementation}

All models were written in BNGL (the BioNetGen modelling language) and developed using RuleBender, an integrated development environment for BioNetGen [84]. All simulations were conducted using the standard deterministic and stochastic simulators included with BioNetGen, although stochastic simulations were only used 


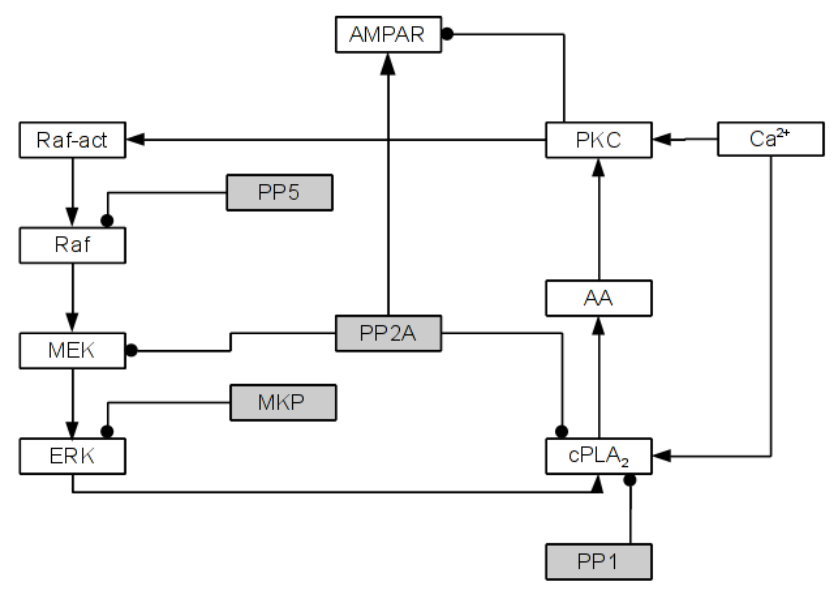

Figure 4.1: Block diagram of the synaptic signal transduction model, based on [3]

in the validation of the synaptic model. Development of the models occurred over three phases: implementation of the synaptic model, implementation of the scaffold model, and merging the two to create the final models for simulation. In the following sections, we discuss each of these in turn.

\subsubsection{Implementation of the Synaptic Model}

Our core synaptic model is the cerebellar long-term depression model described by Antunes and De Schutter [3]. Figure 4.1 presents a block diagram of the signalling network. The full original model consists of 102 chemical species and 207 reactions, all of which are included in the supplementary material along with rate constants and initial concentrations. All of the basic information required for replication is thus available. Implementing the model in BioNetGen, however, requires some additional work. Section 2.4.2 provides an explanation of BioNetGen, but we discuss some of the pertinent implementation issues here. 
The first step was to develop representations for each of the basic molecule types. In this case, we have several types of proteins (enzymes, scaffolds, and receptors) and two small molecules (calcium and arachidonic acid). Small molecules require only minimal internal structure and do not pose a problem. The representation of proteins requires more attention. The general recommendation is to name the components of a protein based on well-established structural features of the molecule, such as domains, binding sites, and phosphorylation sites (usually denoted by amino acid positions) [82]. We used the online protein sequence and functional annotation databases UniProt [25] and SMART [56] to determine which domains and other features were involved in the model reactions and how they needed to interact. Fortunately, Antunes and De Schutter provide considerable detail concerning the exact protein isoforms, structural features, and reaction mechanisms included in their model, which greatly facilitated the process of developing representations. We used UniProt to identify the binding sites and phosphorylation sites on each protein, and consulted SMART for the domain structure. In principle, either database could have provided all of the necessary information, but UniProt tends to have more information on functional interactions while SMART provides a much more consistent and well-established naming scheme for protein domains (as well as graphical representations for each entry). Only those domains and sites that are directly involved in model reactions were included in our representations. In a few cases, minor deviations from a purely structural representation were required to accommodate the necessary reactions.

Figure 4.2 presents two examples of a graphical representation from SMART and its corresponding BNGL representation. Part A shows the representation for ERK. 


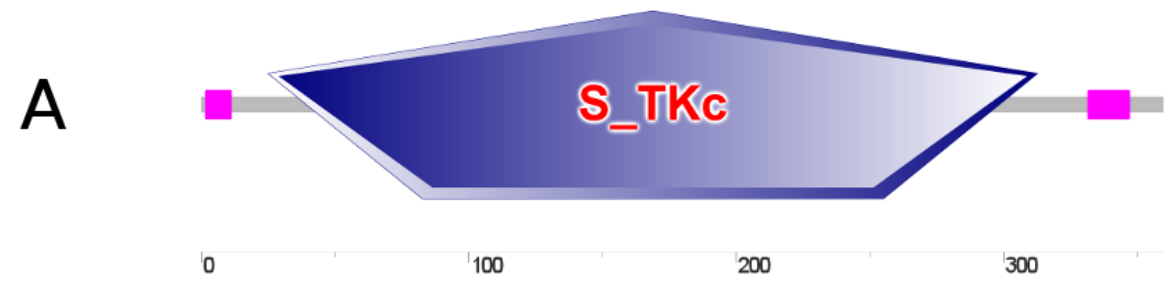

\section{$\operatorname{ERK}\left(T 185 \sim U \sim P, Y 187 \sim U \sim P, S \_T K c\right)$}

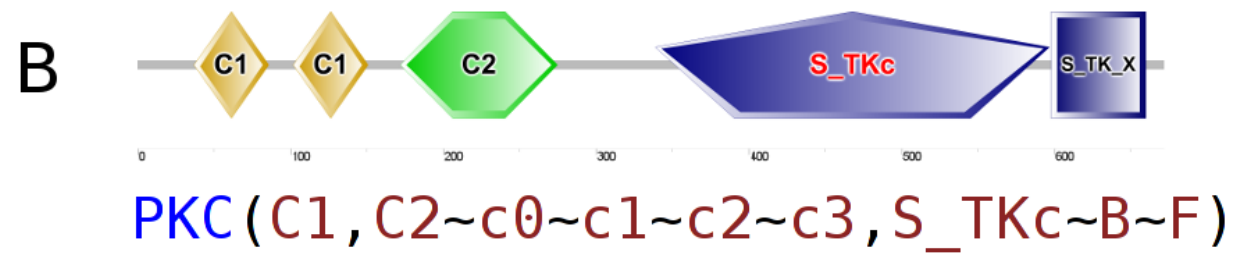

Figure 4.2: Examples of protein representation: comparison of the graphical representation from the SMART database and its corresponding representation in BNGL for the enzymes ERK (part A) and PKC (part B)

SMART reveals that the only conserved domain for this protein is the serine/threonine kinase core (the catalytic domain), which is designated ' $\mathrm{S} \_T K c$ '. The enzyme is activated by two phosphorylation events, which UniProt reports as occurring at amino acid positions 185 and 187 (threonine and tyrosine residues, respectively). Each of these sites have two states ( $\mathrm{U}$ and $\mathrm{P}$ ) representing whether or not they are phosphorylated; the catalytic domain does not have states and is only used to bind other molecules upon which the enzyme will act. This simple representation provides all the structural components needed for reactions involving ERK in the model. Part $\mathrm{B}$ shows the more complicated case of PKC. The activation of PKC is highly complex and there are many forms of the active enzyme ( 4 of which are included in the model). The key to our representation is that the kinase core $\left(\mathrm{S}_{-} \mathrm{TKc}\right)$ is bound internally when the molecule is inactive, but is freed upon activation. We represent this by 
two states (B and $\mathrm{F}$ ) on the kinase core. The remaining domains bind the activators of PKC, which include $\mathrm{Ca}^{2+}$, $\mathrm{AA}, \mathrm{DAG}$, and phorbol esters. Of these, only $\mathrm{Ca}^{2+}$ and AA are considered in the model. The $\mathrm{C} 2$ domain binds three calcium ions, which we represents using states instead of bonds because BioNetGen only allows one bond per component. The $\mathrm{C} 1$ domains can bind any of the other activators, but we only need one of the two domains to bind a molecule of AA; the other $\mathrm{C} 1$ domain is not represented. (The 'S_TK_X' domain in the SMART representation is an extension of the catalytic domain and is not necessary in the model). The rest of the base molecule types were represented similarly.

The second major step in the implementation of the synaptic model was to convert the reactions from the original into rules in BioNetGen. The general recommendation in writing rules is to specify only the components of the reactants that affect the rate of the reaction [82]. However, our goal was to match the original model as closely as possible, so our approach deviated from this recommendation to some extent. Most of our rules fully specify the chemical species involved to ensure that the model generates the exact number of species and reactions found in the original. In the original model, each of the 207 reactions is written out explicitly. Our BioNetGen implementation accomplishes the same number of reactions using 95 rules for two main reasons. First, BioNetGen allows bidirectional rules for binding reactions, whereas the STEPS simulator used in the original does not. Second and more importantly, BioNetGen's use of pattern matching in rules allowed us to efficiently represent cases where multiple forms of a molecule are involved in reactions that have the same rate constants. For instance, all four of the active forms of PKC in the model are assumed to have the same activity and will catalyze a given reaction with the same rate; these are written 
out separately in the original, but we can represent them all with a single BioNetGen rule because we can specify all active forms with the pattern $\mathrm{PKC}\left(\mathrm{S}_{-} \mathrm{TKc} F\right)$. There were several such cases in the original model that could be represented with one or two rules in BioNetGen.

In order to validate the completed BioNetGen implementation of the synaptic model, we followed the same procedure used to validate the original model. In that case, Antunes and De Schutter validated their model against the experimental results of Tanaka et al. [92], who meticulously studied the calcium requirements for the induction of cerebellar long-term depression. Antunes and De Schutter simulated the experimental conditions of Tanaka et al. and then performed similar analyses to verify that their model reproduced the essential properties observed in real neurons. Using our model, we followed the same procedure and verified that our implementation accurately reproduces the original results. Further details on validation procedures and results are presented in Appendix A.

\subsubsection{Implementation of the Scaffold Model}

Our core scaffold model is the model described by Kocieniewski et al. [49], which consists of the basic MAPK cascade and the scaffold protein most closely associated with it in mammals, KSR. Figure 4.3 shows a block diagram of the model. The original model was written in BioNetGen and the authors report a total of 20 rules and 15 parameters, which generate a total of 495 reactions involving 85 chemical species. All of the parameters are included in the paper, along with a model file written in BNGL. Our goal was to merge the reactions of this scaffold model into our implementation of the synaptic model as an efficient means of adding the KSR to the 


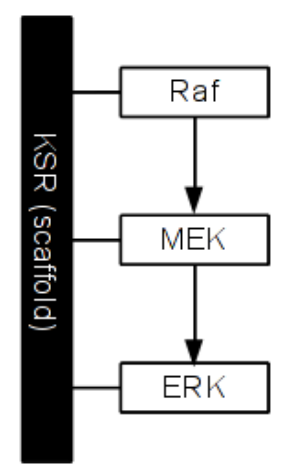

Figure 4.3: Block diagram of the core scaffold model, based on [49]

synaptic model. However, we needed to modify some of the reaction mechanisms used in the scaffold model so that they matched and could be merged with the synaptic model. These changes were implemented incrementally over five stages, starting with a basic implementation of the Kocieniewski model and ending with a model that met our two goals. In the following paragraphs, we describe these five stages.

The first stage was simply to implement the scaffold model exactly as described in the original paper. Since we ultimately wanted to merge this model with the synaptic model, we used the protein representations created previously. Thus, the reactions of the MAPK cascade were implemented using the Raf, MEK, and ERK representations from the synaptic model. Aside from using our own representations, everything else in this model is the same as in the original. We then simulated our version under the same conditions used in the original to ensure that it was implemented correctly; we found that our results matched the original results very closely. With this model constructed and validated, we could begin making the necessary modifications. Each of the following stages involved making a single type of modification and rerunning the test simulations to make sure the basic responses and properties were retained. 
In the second stage, we replaced cytoplasmic enzymatic reactions. In the original model, reactions that occur in the cytoplasm are represented by simple second-order reactions of the form $\mathrm{E}+\mathrm{S} \stackrel{k_{c} a t}{\longrightarrow} \mathrm{E}+\mathrm{P}$. Here, we have a single reaction step and a single rate constant to represent the reaction, which ignores the formation of the enzyme-substrate complex. Since our synaptic model used the elementary reaction step approach exclusively, we needed to change these reactions to have the form $\mathrm{E}+\mathrm{S} \underset{k_{b}}{\stackrel{k_{f}}{\rightleftharpoons}} \mathrm{E} \cdot \mathrm{S} \stackrel{k_{c} a t}{\longrightarrow} \mathrm{E}+\mathrm{P}$; in other words, we needed to add the steps for the formation and dissolution of the enzyme-substrate complex. The simplest and most consistent way to do this was to replace the cytoplasmic reactions in the scaffold model with the corresponding reactions from the synaptic model. In this process, we also used the synaptic rate constants for each reaction step. This ensured that the cytoplasmic reactions in our scaffold model were identical to those in our synaptic model, which is essential for merging them.

Next, we changed the way dephosphorylation was represented in the scaffold model. The original model does not include phosphatases to dephosphorylate the activated kinases, but rather assumes that phosphorylated states decay passively. Again, this approach is incompatible with the synaptic model, which includes several phosphatases and, as always, models their activity using elementary reaction steps. As in the previous stage, our solution here was simply to replace the dephosphorylation reactions in the scaffold model with those from the synaptic model, again using the rate constants from the latter. The fourth stage was simply to change the initial concentrations for all molecules in the scaffold model to match those in the synaptic model. With this change, the two models were essentially ready for merging: all shared reactions, rate constants, and initial concentrations were now identical. 

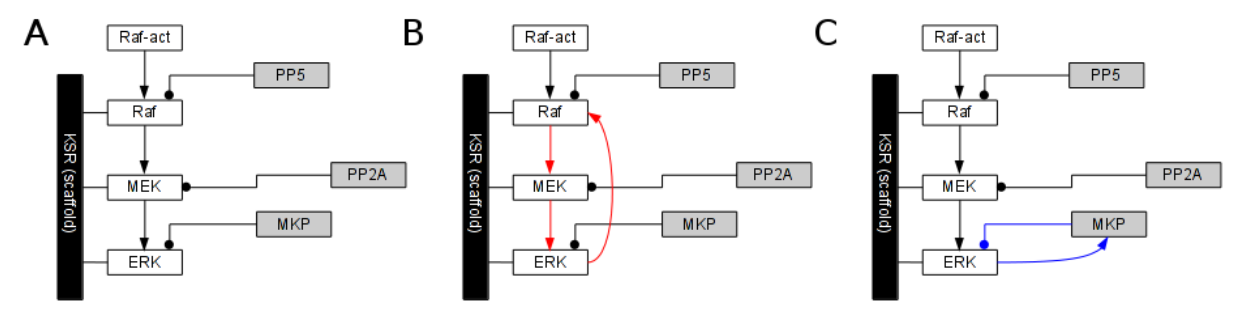

Figure 4.4: Three versions of the pathway model: A) no feedback, B) positive feedback, and $\mathrm{C}$ ) negative feedback

However, as noted in Section 3.2.4, Kocieniewski et al. assumed a rate constant for catalytic steps of enzymatic reactions on the scaffold that was much higher than their cytoplasmic counterparts, and this may be one reason why they observed such strong effects of the scaffold on activation thresholds as compared to the cytoplasmic case. Therefore, the fifth and final stage of our scaffold model development was simply to set the catalytic rates on the scaffold equal to their cytoplasmic counterparts.

As a final note on the scaffold model, it is important to mention that Kocieniewski et al. used processive scaffold reactions in their model. We also implemented distributive scaffold reactions to explore how this difference affects activation thresholds. In the original scaffold model, the enzymes remain bound to the scaffold during the catalytic step. To implement distributive reactions, the only change required is simply to modify the catalytic step of scaffold reactions so that the bonds between the enzymes and scaffold are broken.

\subsubsection{Creation of Final Models}

The last phase of model development was to add feedback loops and merge the scaffold and synaptic models. We added the feedback loops to the scaffold model before 

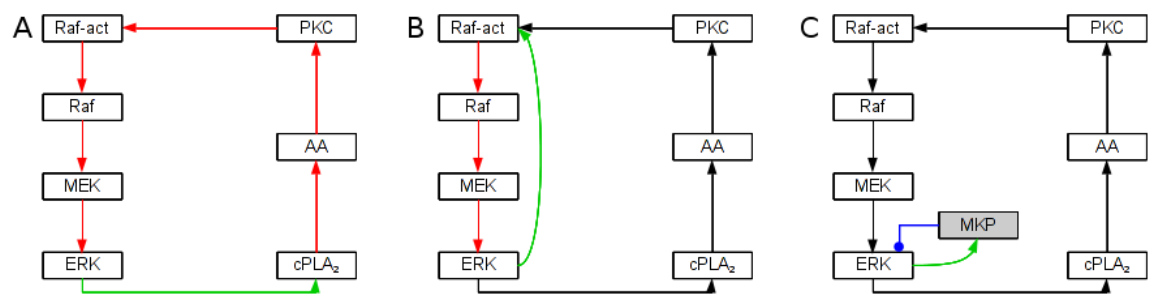

Figure 4.5: Three versions of the network model: A) no added feedback, B) positive feedback, and C) negative feedback

merging so that we could compare the results in two contexts: a single signalling pathway based on the scaffold model and a larger signalling network based on the synaptic model. In order to avoid confusion and to distinguish them from their precursors, we refer to these models as the pathway model and the network model, respectively. We selected two feedback loops from the literature reviewed in Chapter 3 to add to the pathway model. The first is a positive feedback loop between ERK and Raf, described by Smolen et al. [87], and the second is a negative loop between ERK and MKP, described by Bhalla [11]. In each case, the original paper provided the necessary reactions and parameters, which were implemented using the same procedures previously described. We added the loops individually to create three versions of the pathway model: one without feedback, one with the positive loop, and one with the negative loop. Figure 4.4) presents block diagrams of these three models.

Next, we merged these three pathway models with the network model. This was a straightforward matter because of the changes we had previously made to the pathway model; all that was required was to copy the parameters and reaction rules related to the scaffold and the feedback loops into the network model. Thus, we had three 
corresponding versions of the network model. It is important to note, however, that the network model already included the MAPK-PLA 2 -PKC positive loop. Figure 4.5 shows the three versions of the network model. 


\section{Chapter 5}

\section{Results and Discussion}

In this chapter, we present the results of experiments conducted on the models described in the previous chapter. First, we tested our scaffold model to ensure that it could reproduce the basic responses of the original; the results of these tests are presented in Section 5.1.1. In Section 5.1.2, we describe the suite of experiments performed on the network model, which explored its responses to various feedback conditions and scaffold affinities, first individually and then combined in the same experiments. We also performed these experiments on the pathway model, and we describe the differences between the network and pathway model responses in Section 5.1.3. Finally, we conclude with a discussion of the overall results and the possible explanations for our observations in Section 5.2. 


\section{$5.1 \quad$ Results}

\subsubsection{Basic Behaviour of the Scaffold Model}

We first determined if our modified scaffold model could reproduce the basic results of previous studies. In particular, we tested for the presence of an optimal range of scaffold concentrations and for an effect of the scaffold on steady-state levels of activated ERK. We also looked into the effects of distributive versus processive scaffold reaction mechanisms, as well as the effects of the location of dephosphorylation events (in the cytoplasm or on the scaffold). We conducted these experiments using the pathway model and compared results from the original version (i.e., the direct implementation of Kocieniewski et al. [49]) and our modified version (which incorporates all of the changes described in Section 4.2.2).

Overall, the behaviour of our implementation of the scaffold model was qualitatively similar to that of the original. Both versions display the characteristic sigmoidal response of the MAPK cascade to varying signal magnitudes in Figure 5.1 and the optimal scaffold concentration range for processive scaffold models in Figure 5.2. The scaffold concentration effects were similar in the original and modified pathway models, with an optimal range between 0.01 and $1.0 \mu \mathrm{M}$ and a peak concentration just above $0.1 \mu \mathrm{M}$. The responses to different signals showed more differences. The bottom plots are clearly shifted to the right of the top plots, indicating that the threshold for activation of the modified models was slightly higher than in the originals. Additionally, the modified processive scaffold model displayed a sharper threshold than the original version, as indicated by the steeper rise of the bottom curve. Under these simple conditions, our scaffold model did not appear to diminish the threshold of the 




B

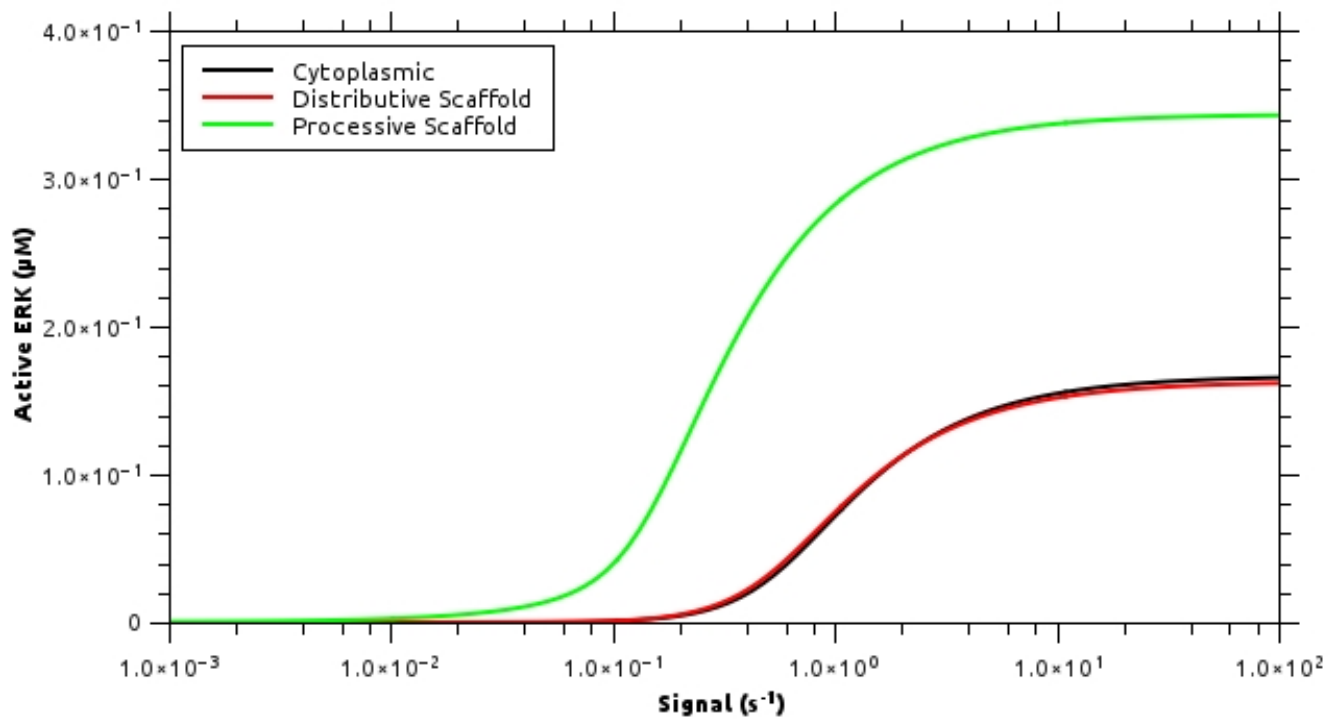

Figure 5.1: Effects of signal magnitude variation. Part A: Original model. Part B: Our implementation. 

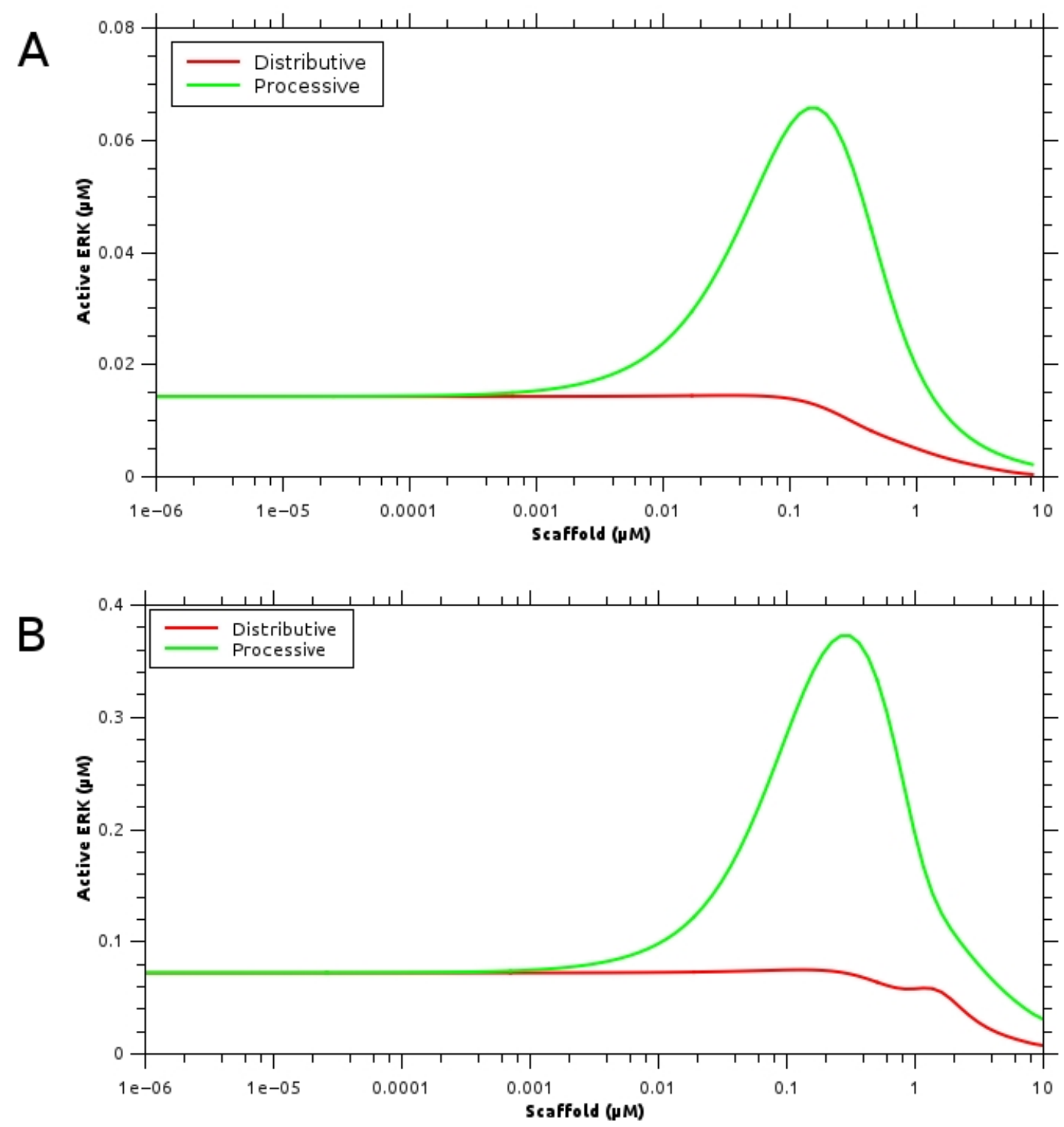

Figure 5.2: Effects of scaffold concentration variation. Part A: Original model. Part B: Our implementation. 


\section{MAPK cascade.}

Figure 5.1 also shows that the distributive scaffold case was nearly identical to the cytoplasmic case in both versions. Moreover, Figure 5.2 indicate that the distributive models did not display an optimum range for scaffold concentrations. These observations are precisely in line with those of Levchenko et al. [57], who concluded that distributive reaction mechanisms make scaffolds essentially ineffectual. Indeed, we tested a distributive scaffold model in most of our experiments and its responses were either very similar to or slightly weaker than the cytoplasmic models in all cases. Therefore, we will hereafter focus on the differences between cytoplasmic and processive scaffold models; unless otherwise indicated, the phrase 'scaffold model' (and the like) will refer exclusively to processive scaffold models.

For one final test of basic responses, we explored the effect of the location of dephosphorylation reactions. In the above tests, we assumed that dephosphorylation only occurs in the cytoplasm and that conformational and/or steric effects prevent phosphatases from acting on scaffold-bound substrates. This type of protection from phosphatases is one of the proposed properties of scaffolds [97]. In accordance with previous studies, relaxing this assumption to allow dephosphorylation both in the cytoplasm and on the scaffold did not affect the qualitative results but simply shifted the response curves downward, making the scaffold case more similar to the cytoplasmic case. For clarity and simplicity, we assumed that dephosphorylation occurred only in the cytoplasm for the remainder of the experiments. These basic experiments demonstrate that our scaffold model is capable of reproducing the core results of the Kocieniewski model, as well as the effects of distributive scaffold reactions observed by Levchenko et al. [57]. Supplementary experiments performed on the pathway 
model are summarized in Appendix B.

\subsubsection{Exploration of the Network Model}

In order to address our problem statement, we conducted a series of experiments on the network model, focusing on variations in feedback strength and scaffold affinity under different stimulus and phosphatase conditions (see Section 4.1.3). While our intention was to develop the scaffold model so that it could be merged into the synaptic model without the need to modify any parameters, our initial tests produced some unexpected results and further calibration proved necessary; we describe this process in the next section, before going on to present our experimental results in detail.

\section{Calibration}

The addition of the scaffold to the network model had a very strong effect-so much so that even the smallest $\mathrm{Ca}^{2+}$ stimuli used $(0.5 \mu \mathrm{M})$ fully activated the network. This indicated that the threshold for activation was so low that the network was effectively always on. Since this is clearly not a biologically plausible result, we concluded that the problem was with the standard scaffold parameter values used in the pathway model. To calibrate the network model, we focused on the scaffold dissociation rate and conducted tests with different values to find one that restored the sigmoidal response of the network. Each test assumed a different dissociation rate

constant between 0.1 (the value from the pathway model) and $1.5 \mathrm{~s}^{-1}$, and delivered 1-second calcium pulses with concentrations from 0.5 and $5.0 \mu \mathrm{M}$. Figure 5.3 displays the results of these tests, which showed that increasing the dissociation rate restored 


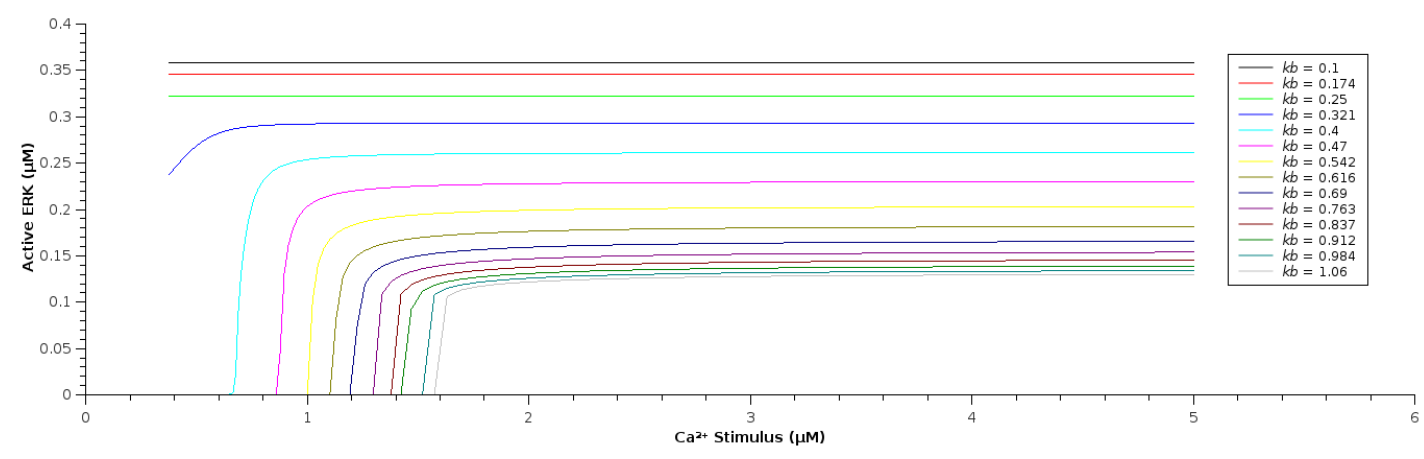

Figure 5.3: Results of calibration tests on the network model. Increasing scaffold dissociation rate constant restored the sigmoidal curve, while increasing the activation threshold and decreasing the maximal response.

the sigmoidal response curve, increased the activation threshold, and decreased the concentration of activated ERK at steady state. We selected the first dissociation rate that restored the sigmoidal curve, which corresponded to a value of $0.4 s^{-1}$ (the cyan curve in Figure 5.3). This value was used as the default dissociation rate constant for all experiments on both the network and pathway models.

\section{Experiments}

In our experiments, we tested the responses of the network model to variations in the four parameters discussed in Section 4.1.3: $\mathrm{Ca}^{2+}$ stimulus concentration, initial phosphatase concentration, feedback strength, and scaffold affinity. We varied these parameters in pairs and tested responses to the following combinations: $\mathrm{Ca}^{2+}$ stimulus versus feedback strength, $\mathrm{Ca}^{2+}$ stimulus versus scaffold affinity, phosphatase concentration versus feedback strength, phosphatase concentration versus scaffold affinity, and finally feedback strength versus scaffold affinity. Parameters and ranges are listed in Table 5.1.2. In all cases, the output of the model was measured as the steady-state 
concentration of activated ERK; it is worth noting that the total concentration of ERK in all of the models was $1.0 \mu \mathrm{M}$, which means that can also consider the plotted concentrations of active ERK as proportions of the total concentration. The results of our experiments are presented as three-dimensional surface plots, which all use the same scale in order to aid in comparison (although the axes were rotated in some cases to provide a better view of the plot). The only exception is experiments involving phosphatase concentration, which produce very strong outputs when the phosphatase concentrations are low and, therefore, use a different scale. However, these phosphatase experiments produce nearly identical results across all modelswhether cytoplasmic or scaffold, pathway or network - and are therefore discussed only briefly.

The results of the $\mathrm{Ca}^{2+}$ stimulus versus feedback strength experiments on the cytoplasmic and scaffold versions of the network model are presented in Figure 5.4. The three parts of this figure show the effects of the three different feedback conditions: standard MAPK-PLA2-PKC feedback (part A), added positive feedback (part B), and added negative feedback (part C). In all of these experiments, we varied the relevant feedback rate constant between 0.01 and $50.0 \mathrm{~s}^{-1}$ and applied $\mathrm{Ca}^{2+}$ pulses for 1 second at concentrations between 0.5 and $5.0 \mu \mathrm{M}$. In both the cytoplasmic and scaffold models, the different feedback loops had little effect on the value of the threshold; in parts $\mathrm{A}$ and $\mathrm{B}$, the threshold was slightly higher in the lower range of feedback rates, and this effect was more pronounced in the scaffold models. In each feedback condition, the presence of the scaffold lowered the activation threshold from approximately $4.2 \mu \mathrm{M}$ in the cytoplasmic models to approximately $1.8 \mu \mathrm{M}$ in the scaffold models. In parts $\mathrm{A}$ and $\mathrm{B}$, the cytoplasmic models had sharper thresholds than the scaffold 
models, although the positive feedback in part B did sharpen the scaffold threshold somewhat. Interestingly, the scaffold had very little effect on threshold sharpness in part $\mathrm{C}$, the negative feedback condition. In all cases, the feedback loop produced most its effects between strengths of 0.01 and $10.0 \mathrm{~s}^{-1}$, with further increases having very little influence on signalling output.

To examine the effect of the scaffold on the magnitude of the signalling output, we calculated the ratio of the cytoplasmic to scaffold maximal responses. In part A, the cytoplasmic response was approximately 0.83 of the scaffold response, indicating a moderate effect of the scaffold. This difference all but disappeared in part B, where the ratio was nearly 0.99 . The reason for the lack of a difference between the cytoplasmic and scaffold models with added positive feedback is that the maximal ERK response under default phosphatase concentrations appears to be approximately 0.42 $\mu \mathrm{M}$; in all of our experiments, the only ones that produced higher concentrations of active ERK were those that lowered phosphatase concentrations, leading to runaway phosphorylation and activation of nearly all of the available ERK. In part C, the ratio of maximal responses was 0.75 ; however, since the maximal responses occurred only at the lowest feedback strengths and dropped off rapidly, the ratio in the plateaus of the plots is more indicative of the overall response. Here, the ratio fell to approximately 0.53 , indicating that the scaffold had the largest effect in the negative feedback condition. This was an unexpected result, but it might have to do with the lower levels of activated ERK allowing greater headroom for the scaffold to enhance signalling.

The results of the $\mathrm{Ca}^{2+}$ versus scaffold affinity experiments are presented in Figure 5.5. These experiments were conducted in a similar manner to the previous set; we 

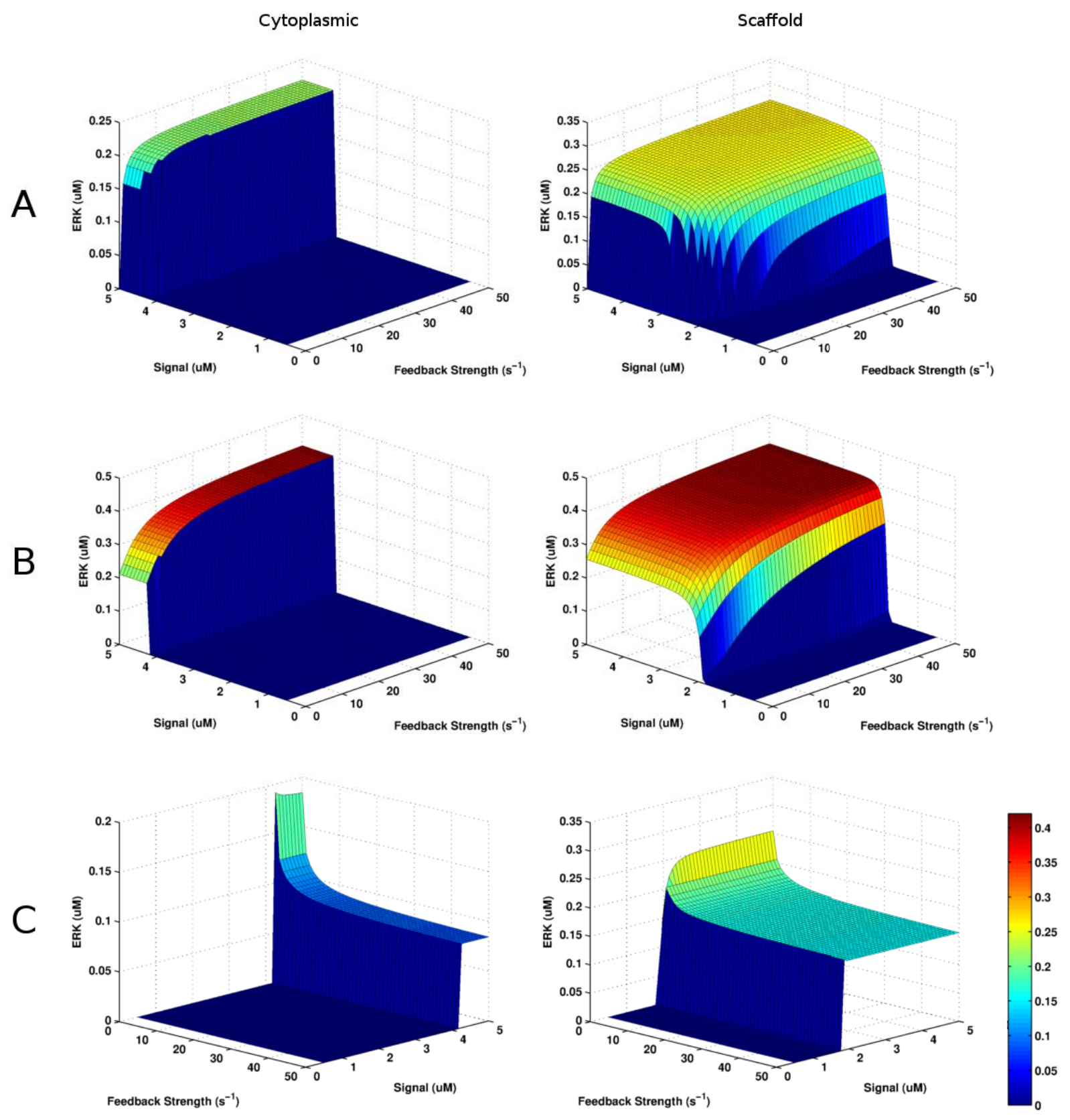

Figure 5.4: Results of $\mathrm{Ca}^{2+}$ stimulus versus feedback strength for the cytoplasmic and scaffold versions of the network model. Part A shows the results with no added feedback; part B with added positive feedback; and part $\mathrm{C}$ with added negative feedback. 

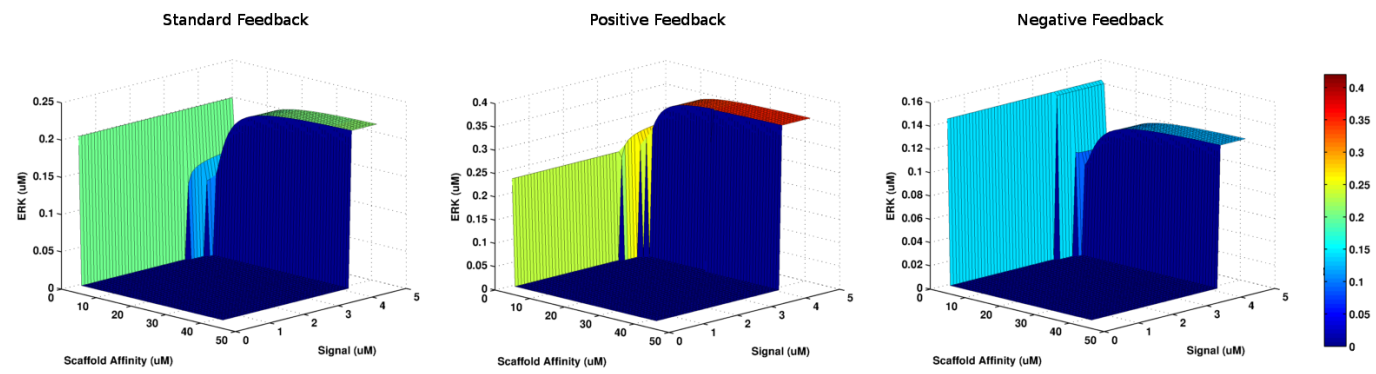

Figure 5.5: Results of $\mathrm{Ca}^{2+}$ stimulus versus scaffold affinity experiments for the network model under the three feedback conditions.

varied the scaffold dissociation rate constant $\left(k_{b}\right)$ between 0.01 and $50.0 \mathrm{~s}^{-1}$ while applying 1-second $\mathrm{Ca}^{2+}$ pulses at concentrations between 0.5 and $5.0 \mu \mathrm{M}$. Each feedback condition displayed a similar complex pattern of responses: strong, constant activation at low $k_{b}$ regardless of stimulus magnitude, followed by several bands of decreasing outputs, and finally a ramp up to a plateau. The constant activation at low $k_{b}$ and the decreasing bands were expected from the calibration results discussed above; the threshold increased as the signalling output decreased, just as during calibration, but the effect is less smooth because the intervals between $k_{b}$ values were larger in these experiments than in the calibration. The ramp up to the plateau likely reflects a redirection of signalling from primarily on the scaffold to primarily in the cytoplasm as the dissociation rate increased and scaffold-mediated signalling became unfavourable.

Figure 5.6 presents the results of the feedback strength versus scaffold affinity experiments. We varied the feedback rates $\left(k_{c a t}\right)$ and the scaffold dissociation rate $\left(k_{b}\right)$ over the same ranges as previous experiments: from 0.01 to $50.0 \mathrm{~s}^{-1}$. Unlike the previous experiments, the feedback strength versus scaffold affinity experiments did not include variations in $\mathrm{Ca}^{2+}$ input as an explicit part of the design. Therefore, 

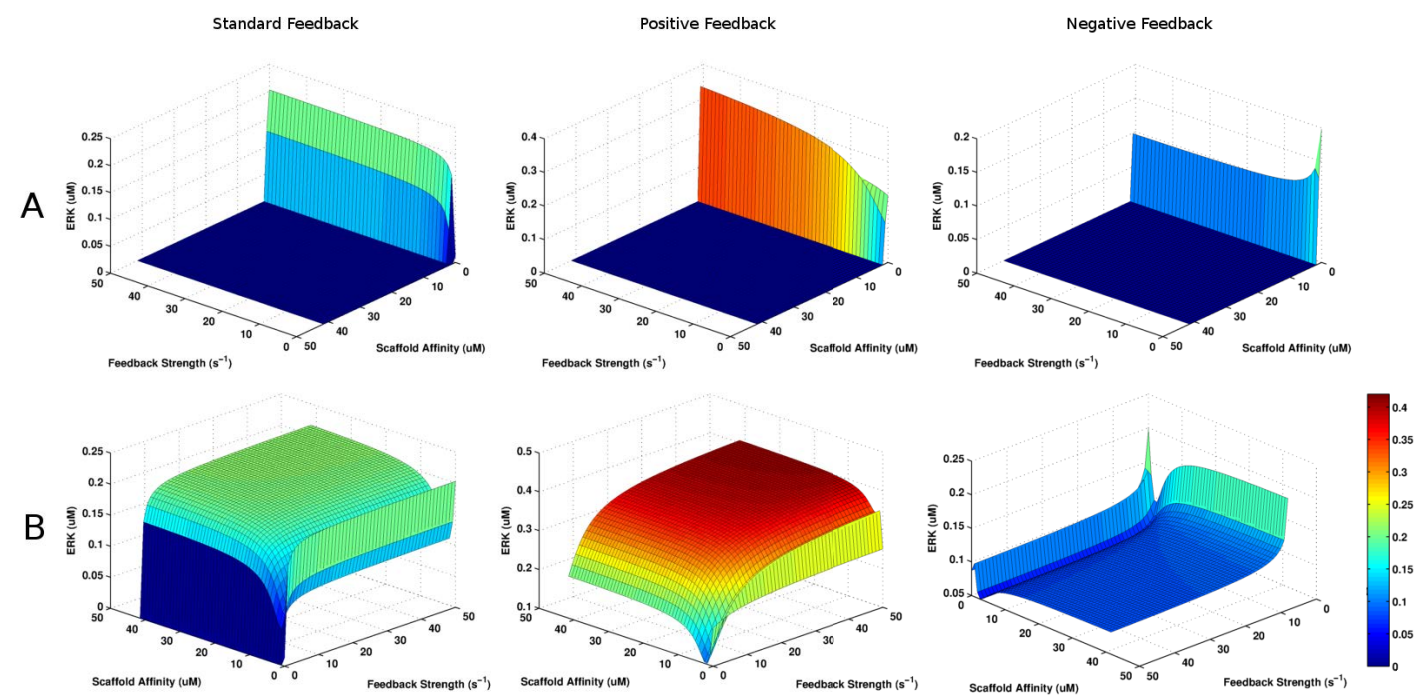

Figure 5.6: Results of feedback strength versus scaffold affinity experiments on the network model under the three feedback conditions. In part $\mathrm{A}$, an intermediate $\mathrm{Ca}^{2+}$ stimulus of $2.5 \mu \mathrm{M}$ was applied, while a strong $5.0 \mu \mathrm{M}$ stimulus was used in part B.

we conducted the experiments twice using 1-second pulses at $2.5 \mu \mathrm{M}$ (part A) and $5.0 \mu \mathrm{M}$ (part B) in order to get a sense of how the system responded to different stimulus levels. These plots are exactly what we would expect on the basis of the previous experiments if there was no significant interaction between feedback and scaffolding in the models. In part A, each feedback condition displayed the strong bar of activation at low $k_{b}$ observed in Figure 5.5, tempered by the variations in feedback strength; indeed, the curves at low $k_{\text {cat }}$ values are very similar to those in Figure 5.4. Likewise, the plots in part B show the same effects of feedback and scaffold affinity superimposed at high stimulus values. In short, there was no discernible interaction between feedback and scaffolding under the conditions of these experiments.

Finally, the experiments involving variations in phosphatase concentration had very little effect, and results across all models were nearly identical. In these ex- 


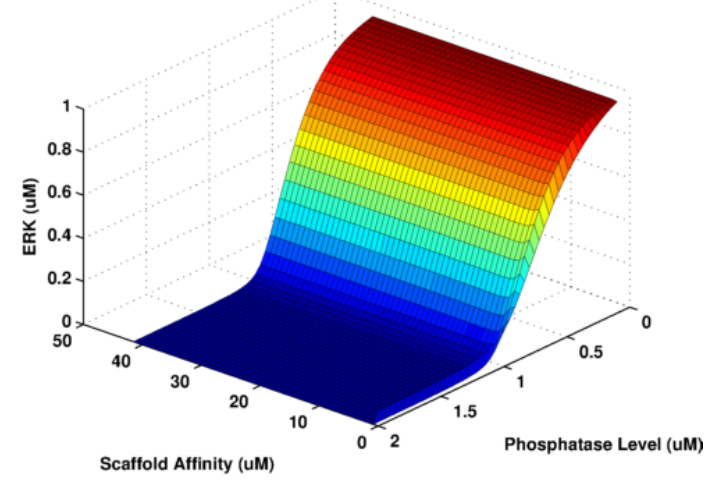

Figure 5.7: Results of an example phosphatase concentration versus scaffold affinity experiment.

periments, we multiplied the initial concentration of all phosphatases (PP1, PP2A, PP5, and MKP) by a factor that varied between 0.1 and 2.0. We conducted two sets of experiments in which we varied this concentration factor in conjunction with feedback strength in one set and scaffold affinity in the other. Figure 5.7 presents an example a phosphatase concentration versus scaffold affinity experiment, stimulated by a 1-second $\mathrm{Ca}^{2+}$ pulse at $2.5 \mu \mathrm{M}$. At concentration factors close to 1.0 , the output response was moderate, as expected from previous results. As the factor decreased, the output increased rapidly, ultimately reaching close to total saturation of the available ERK. Increasing the factor beyond 1.0 rapidly reduces the output to 0 . Thus, the model is very sensitive to phosphatase levels, tending either to runaway phosphorylation or full suppression of signalling.

\subsubsection{Comparison of the Pathway and Network Models}

We conducted the same set of experiments described in the previous section on the pathway model to explore their differences and determine if the lack feedback- 


\begin{tabular}{|l|l|}
\hline Parameter & Range \\
\hline \hline Pathway Stimulus & $0.01-10.0 \mathrm{~s}^{-1}$ \\
\hline Network Stimulus & $0.5-5.0 \mu \mathrm{M}$ \\
\hline Scaffold Dissociation Rate & $0.01-50.0 \mathrm{~s}^{-1}$ \\
\hline Feedback Rate & $0.01-50.0 \mathrm{~s}^{-1}$ \\
\hline Phosphatase Concentration & $0.1-2.0 \mu \mathrm{M}$ \\
\hline
\end{tabular}

Table 5.1: Parameters and ranges for all experiments on the pathway and network models. The only parameter that differs between the pathway and network models is the stimulus, where the pathway model uses a rate-based constant stimulus and the network model uses a concentration-based 1-second pulse of $\mathrm{Ca}^{2+}$. Scaffold dissociation rates are the same for all kinases (Raf, MEK, and ERK). The Feedback Rate parameter range listed applies to all feedback loops in both models, although the reaction to which it is applied differs; specific reactions are provided in Section 4.1.3. Phosphatase concentrations apply to all phosphatases in both models (PP2A, PP5, and MKP), although PP1 is present only in the network model.

scaffolding interaction observed in the network model also occurred in the pathway model. In order to allow for direct comparison, all of these experiments on the pathway model used the scaffold dissociation constant derived during calibration of the network model. The pathway model did not strictly require this change to produce a reasonable result, and introducing it merely lowered the outputs without affecting the qualitative behaviour. Nonetheless, the effect of the scaffold on the pathway model was quite different from the network model, despite the fact that the core reactions are the same. There are, however, important differences between the two models. The pathway model contains only the MAPK cascade, while the network model also includes the PKC pathway and the feedback loop connecting them. Additionally, the stimuli in the pathway model are constant and modelled as simple first-order reactions acting on the 'Raf-act' molecule (see Figure 4.4), while the network model stimuli are brief pulses of $\mathrm{Ca}^{2+}$ that activate PKC, which in turn activates Raf-act. Thus, the 

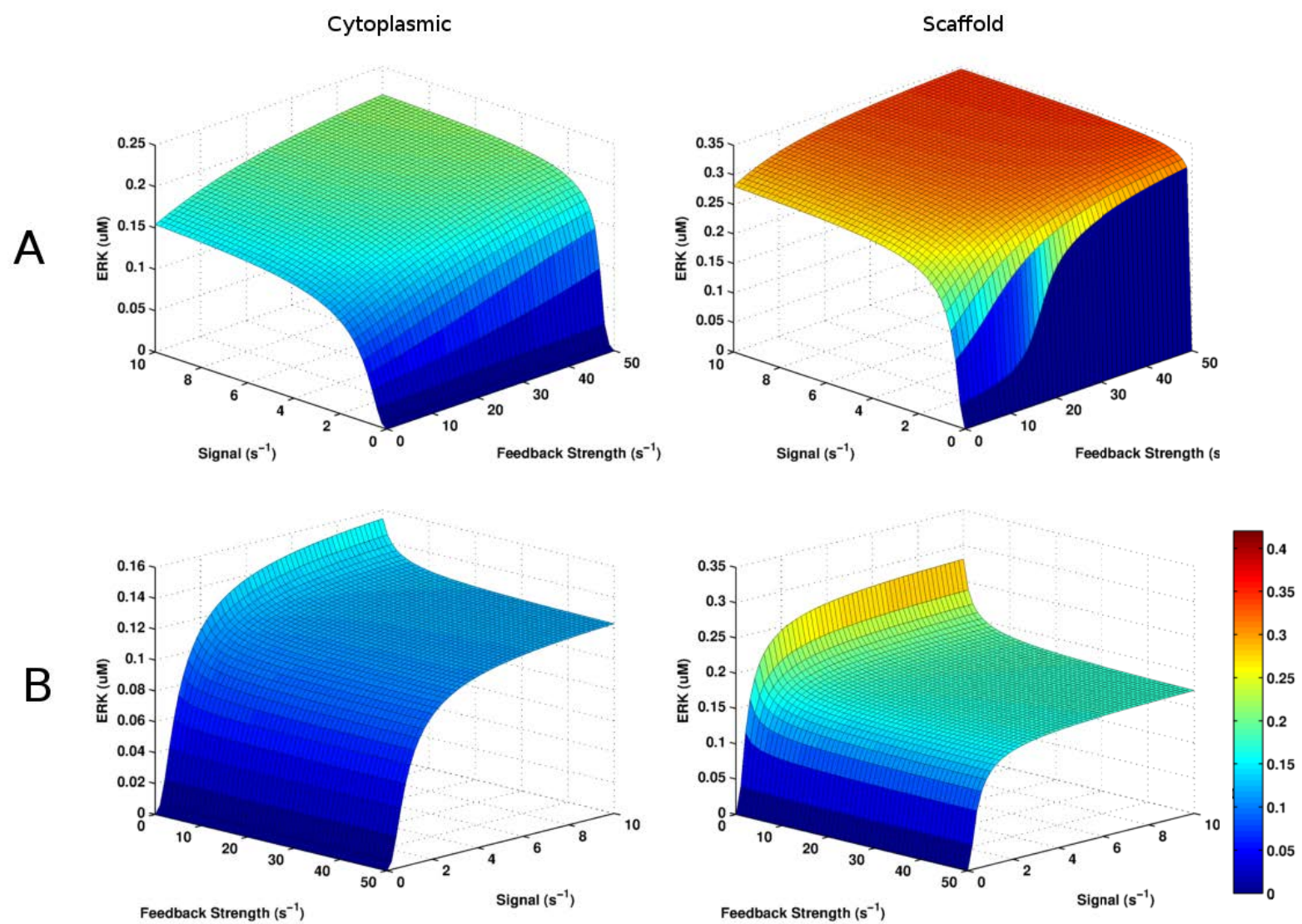

Figure 5.8: Results of input stimulus versus feedback strength for the cytoplasmic and scaffold versions of the pathway model. Parts A and B show the positive and negative feedback conditions, respectively.

network and pathway models differ in size, stimulus type, and upstream activation steps for the MAPK cascade. In terms of experimental manipulations, the only difference between the experiments in the previous section and those described below is in the range of stimulus values; the pathway experiments use a stimuli between 0.01 and $10.0 \mathrm{~s}^{-1}$, which is a slightly smaller range than that used by Kocieniewski et al. in their study [49].

Figure 5.8 presents the results of the feedback versus stimulus experiments on the 

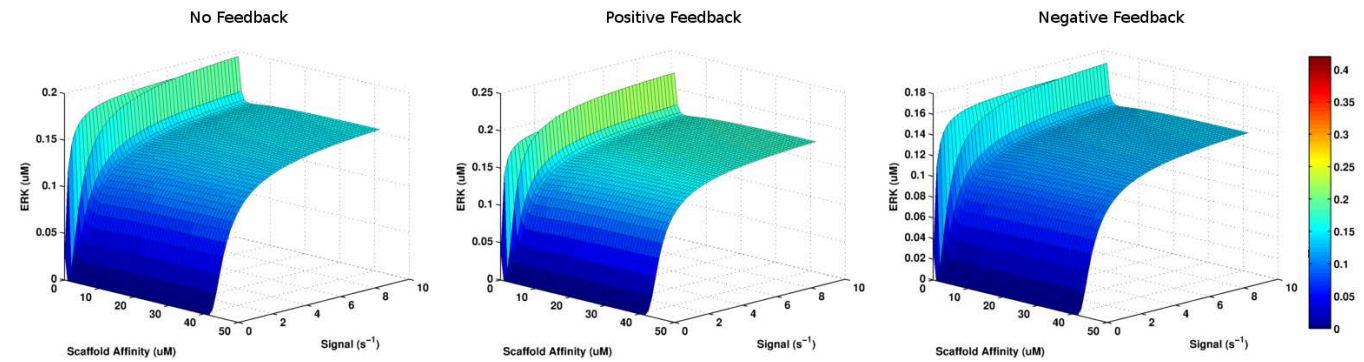

Figure 5.9: Results of input stimulus versus scaffold affinity experiments for the pathway model under the three feedback conditions.

pathway model, comparing the cytoplasmic and scaffold versions with added positive feedback (part A) and negative feedback (part B). In both feedback conditions, the scaffold models had sharper thresholds than their counterparts, but did not appear to shift the position of the thresholds very much, if at all. These results are essentially opposite those observed for the network model. Moreover, the effect of the scaffold on the magnitude of the ERK output showed a different pattern than the network model. In part $\mathrm{A}$, the ratio of cytoplasmic to scaffold maximal responses was approximately 0.63, indicating a fairly strong effect of the scaffold. Likewise, the ratio in the plateaus of part B was close to 0.66 . Thus, the scaffold had approximately the same effect on signalling regardless of whether the feedback was positive or negative. Again, these results effectively reverse those of the network model. Of course, such comparisons are complicated by the fact that the pathway model does not have a default feedback loop, as the network model does. Nonetheless, they do demonstrate that the effect of the scaffold is highly sensitive to the context of signalling.

Figure 5.9 presents the results of the stimulus versus scaffold affinity experiments for the pathway model. Here again, the pattern of responses was quite different from that observed for the network model. As expected, the responses were strongest for 
the lowest values of dissociation rate, but they all displayed a clear threshold in terms of stimulus strength. Moreover, increasing the dissociation rate did not produce the progressive drop followed by a sharp increase in responses that characterized the network model. However, the pattern of responses was consistent across the three feedback conditions, just as it was in the network model. It is interesting to note that the different feedback conditions had a much more modest effect on the magnitude of signalling outputs compared to the network model. This difference is likely due to the fact that the network model has a default positive feedback loop (the MPPL) that could interact with the added loops, whereas the pathway model does not. On the other hand, even the MPPL on its own had a much stronger effect on signalling than the positive feedback in the pathway model considered alone.

Finally, the results of the feedback strength versus scaffold affinity experiments are presented in figure 5.10. As for the network model, we performed these experiments twice: once with an intermediate input stimulus at $1.0 \mathrm{~s}^{-1}$ (part A) and once with a strong stimulus at $10.0 \mathrm{~s}^{-1}$ (part B). These plots are largely consistent with the notion that they reflect a simple combination the previous results. For the positive feedback condition, however, there is little difference between the two different stimulus conditions in terms of the peak responses. While this result is in line with the responses in 5.9 for the signal versus scaffold affinity experiments, it is interesting that the positive feedback, particularly under the strong stimulus in part B, was not able to drive the peak response anywhere near the levels observed in the stimulus versus feedback experiments (Figure 5.8). In contrast, the positive feedback in the network model was able to produce peak responses that were very similar to those in other experiments. On the basis of these results, it appears that, for the pathway 

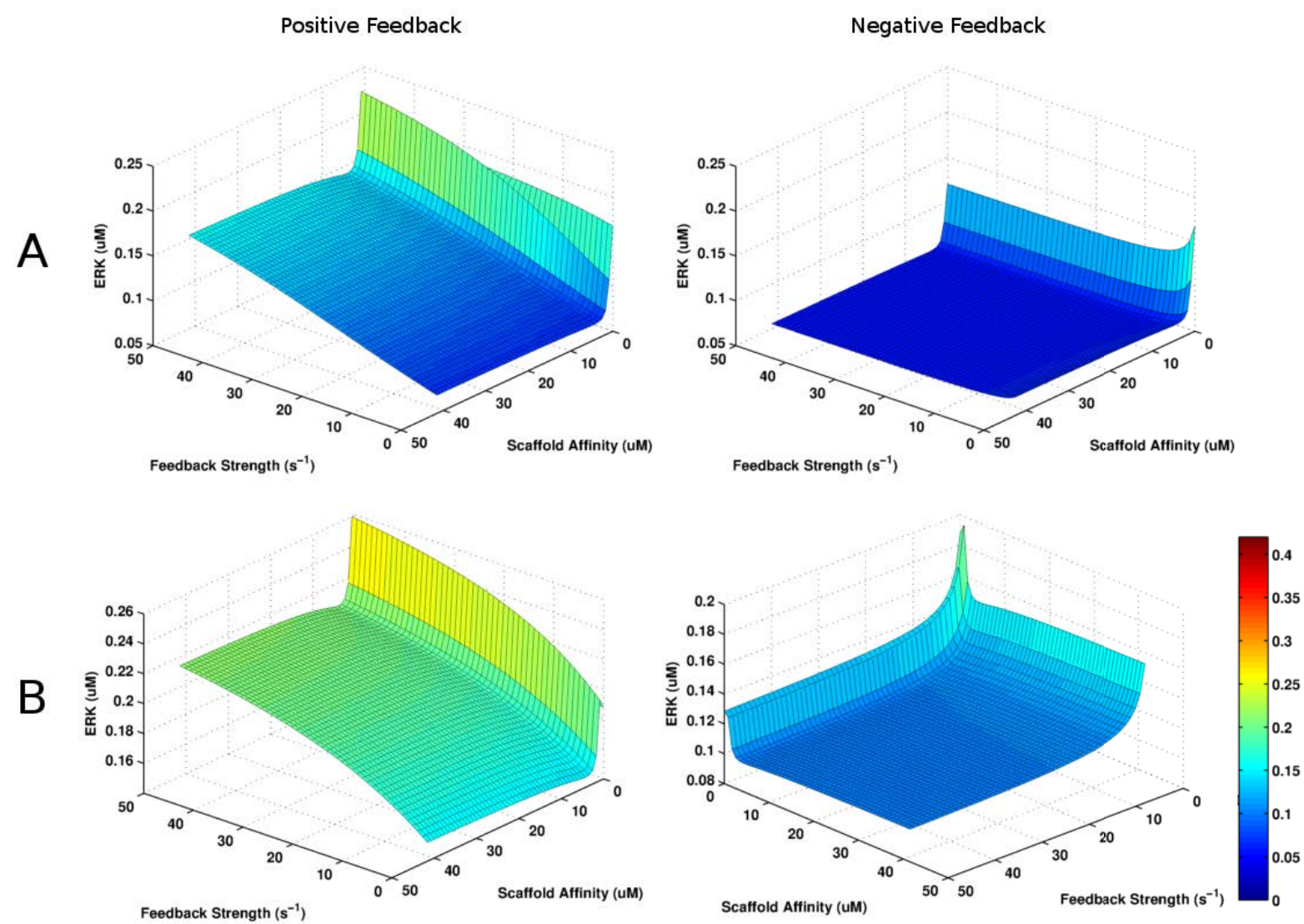

Figure 5.10: Results of feedback strength versus scaffold affinity experiments for the pathway model under positive and negative feedback conditions. In part A, a moderate stimulus of $1.0 s^{-1}$ was applied, while part B uses a strong stimulus at 10.0 $s^{-1}$. 
model, variations in positive feedback strength are less effective in driving signalling outputs when combined with variations in scaffold affinity than when feedback is considered alone. The results of the pathway and network models are more consistent in the negative feedback condition; in both cases, the plots strongly resemble the individual feedback and affinity results superimposed with comparable peak and plateau responses.

\subsection{Discussion}

The experiments described above have explored the properties of our pathway and network models. Our experiments aimed to determine the qualitative effects of feedback and scaffolding in the induction of synaptic plasticity. Thus, our primary focus was on the network model, which is based on the synaptic model of Antunes and De Schutter. These experiments first explored the effects of feedback and scaffolding independently, and then considered them together to look for an interaction. If there was no such an interaction between feedback and scaffolding, we expected the results of these combined experiments to resemble a simple merging or superimposition of the individual results; on the other hand, if there was a significant interaction, we expected the combined results to diverge from a simple merging. Since the results of our

feedback strength versus scaffold affinity experiments on the network model strongly resemble the individual results superimposed, we must conclude that there was no interaction between feedback and scaffolding under the conditions tested. We also performed the same experiments on the pathway model for comparison, and found no convincing evidence of an interaction here either. The one potential exception was that positive feedback appeared to have less of an impact on signalling when 
combined with variations in scaffold affinity than it did on its own. However, this isolated difference is more likely to be an anomaly than evidence of an interaction.

Why did these experiments fail to provide evidence of an interaction between feedback and scaffolding? One possibility is that the manipulations that we used were too simple to support such an interaction. That is, we could have controlled feedback strength by varying the association and/or dissociation rate constants for enzymes in addition to the catalytic rate constants; likewise, we could have controlled scaffold affinity by varying the scaffold association rate constant in addition to the dissociation rate constant. The advantage of modelling these elementary reaction steps is that we can have finer-grained control of each reaction, but the disadvantage is that it would require much more experimentation to determine appropriate combinations and ranges of values. Our approach was to keep the manipulations simple, but it is possible that they were actually too simple. Moreover, it could be that we were looking for an interaction in the wrong place. Our analysis focused on the magnitude of signalling outputs, but the temporal properties, such as the speed and duration of signalling, can also be important. Further experimentation and analysis that focused on the speed and/or duration of signalling outputs might reveal a temporal interaction. A final explanation for our inconclusive results might be that an interaction between feedback and scaffolding is mediated by the active mechanisms of scaffold function (see Section 2.3.1). For instance, some scaffolds do, in fact, exert an influence on the catalytic activities of bound enzymes, making the catalytic rates on the scaffold and in the cytoplasm unequal. As discussed in Section 2.3.2, the KSR scaffold associated with the MAPK cascade exerts several such influences that could very well facilitate a feedback-scaffolding interaction. Additionally, enzymes often dynamically regulate 
scaffolds through (de)phosphorylation, which can (de)activate the scaffold and/or trigger translocation to different parts of the cell; if one such enzyme is involved with one of the feedback loops, this could also facilitate an interaction. KSR is known to undergo such dynamic regulation, but not all of the enzymes involved have been identified $[52,95]$.

While our experiments did not provide evidence of an interaction between feedback and scaffolding, they did reinforce previous results suggesting that the effect of a scaffold protein is highly sensitive to differences in signalling context. In our pathway model, the scaffold sharpened activation thresholds under all feedback conditions, but had little effect on the placement of those thresholds in terms of the stimulus required. In contrast, the network model produced the opposite results: the scaffold both lowered the thresholds and decreased their sharpness - except in the negative feedback condition, where was only lowered but its sharpness was not affected. It is not clear which of the differences between the pathway and network models are responsible for these different response patterns, but the primary possibilities include: the pre-existing positive feedback loop in the network model, the differences in stimuli, and the differences in the upstream activation steps for the MAPK cascade. Of these three, only the effects of upstream activation steps have been examined in previous research. Thalhauser and Komarova [93] found that they could convert bistable responses into graded, proportional responses (and vice versa) simply by adding, removing, or changing the rates of upstream activation steps for the MAPK cascade. Our results are less extreme, but our experiments did not include any modifications of the upstream events; the differences simply exist between the pathway and network models. 


\section{Chapter 6}

\section{Conclusions and Future Work}

\subsection{Conclusion}

The goal of this thesis was to study qualitative effects of feedback loops and signalling complexes in the induction of synaptic plasticity from a systems biology perspective. In order to meet this goal, we first implemented two core models, one each from the fields of synaptic plasticity and scaffold-mediated signalling (Section 4.2). We then developed a series of models based on the core network and pathway models to produce versions with and without scaffolds, and with different positive and negative feedback loops (Section 4.2.3). Using these models, we performed a variety of experiments that were designed to examine the effects of varying the strength of feedback connections and the affinity with which enzymes bind the scaffold. These experiments aimed to establish the effects of feedback strength and scaffold affinity individually and in conjunction. We were particularly interested in how these different conditions would affect the switch-like, bistable response of the system that plays an important role in the induction of synaptic plasticity. When we performed the 
experiments on the network model, we found that the scaffold tended to lower the activation threshold while reducing its sharpness relative to the cytoplasmic versions, making the system easier to activate but less switch-like (Section 5.1.2). In contrast, performing the same experiments on the pathway model produced nearly opposite responses: the scaffold tended to sharpen activation thresholds but with little, if any, effect on the stimulus magnitude required for activation (Section 5.1.3). When we performed the experiments that varied feedback strength and scaffold affinity in conjunction, however, we found that neither the network model (Section 5.1.2) nor the pathway model (Section 5.1.3) provided evidence of an interaction between feedback and scaffolding.

\subsection{Summary of Contributions}

The primary contributions of this thesis are:

- Synthesis and extension of existing models using recently developed methods (Section 4.2)

- Development of flexible domain-oriented representations for all proteins involved (Section 4.2.1)

- Confirmation of the basic effects of scaffolds in MAPK signalling (Section 5.1.1)

- Further investigation into the complex effects of scaffolds on thresholds (Sections 5.1 .2 and 5.1.3)

- A first examination of the effects of feedback loops and signalling complexes in the same model (Sections 5.1.2 and 5.1.3) 


\subsection{Future Work}

There are several directions that future work on the topic of this thesis could take. The first issue to address is the lack of a discernible interaction between feedback and scaffolding in our experimental results. Another question our work leaves open is the source of the different effects that the scaffold had on the pathway and network models. Finally, our models and methods could be extended in a number of ways. In the following sections, we discuss each of these areas in turn.

\subsubsection{Continuing the Search for a Feedback-Scaffolding In- teraction}

In Section 5.2, we discussed several possible explanations for the lack of an interaction between feedback and scaffolding in our results. One could address each of those issues by continuing to apply the systems biology approach. Recall that this approach is intended to be an iterative process in which we add detail to our models and methods until we observe the properties or behaviour of interest. Our goal in this thesis was to apply a single iteration of the process. Since we did not observe the behaviour of interest, we would continue adding detail. Thus, the next iteration might focus on controlling feedback strength and scaffold affinity by manipulating more than a single parameter for each, which would allow finer-grained control of the entire reaction process. Another iteration might focus on temporal dynamics, and design experiments and analyses to explore the speed and duration of signalling. Either of these could be implemented without requiring major modification of the underlying models, if any at all. If an interaction was still not observed, one could move on to 
implementing some of the active properties of scaffolds - although this would be much more difficult, not only because many of the details are currently unknown, but also because the reactions themselves are more complex. While it is currently unknown if the KSR scaffold exerts any modulatory influence on the catalytic activity of bound enzymes, it is known that the molecule undergoes dynamic regulation in the form of (de)phosphorylation and translocation events. One could begin by allowing the scaffold to be (de)activated by (de)phophorylation, and progress to adding reaction compartments to represent different cellular locations as necessary. Again, the essence of the systems biology approach is that one should continue this process until the behaviour of interest is observed, so long as there is sufficient experimental data to support it.

\subsubsection{Determining the Differences Between the Pathway and Network Model Responses}

The addition of the scaffold to the pathway and network models produced quite different effects in each case, particularly in terms of the properties of the response threshold. In Section 5.2, we identified three potential sources of these differences: the default positive feedback loop in the network model, differences in stimuli, and differences in upstream activation steps. All of these differences could be investigated by continuing the incremental development process by which we transformed the original Kocieniewski et al. scaffold model into the pathway model that we used in our experiments. The challenge here would be to add molecules and reactions to the pathway model in such a way as to address only one difference at a time, ultimately transforming it into the network model. One sequence of steps to achieve this is 

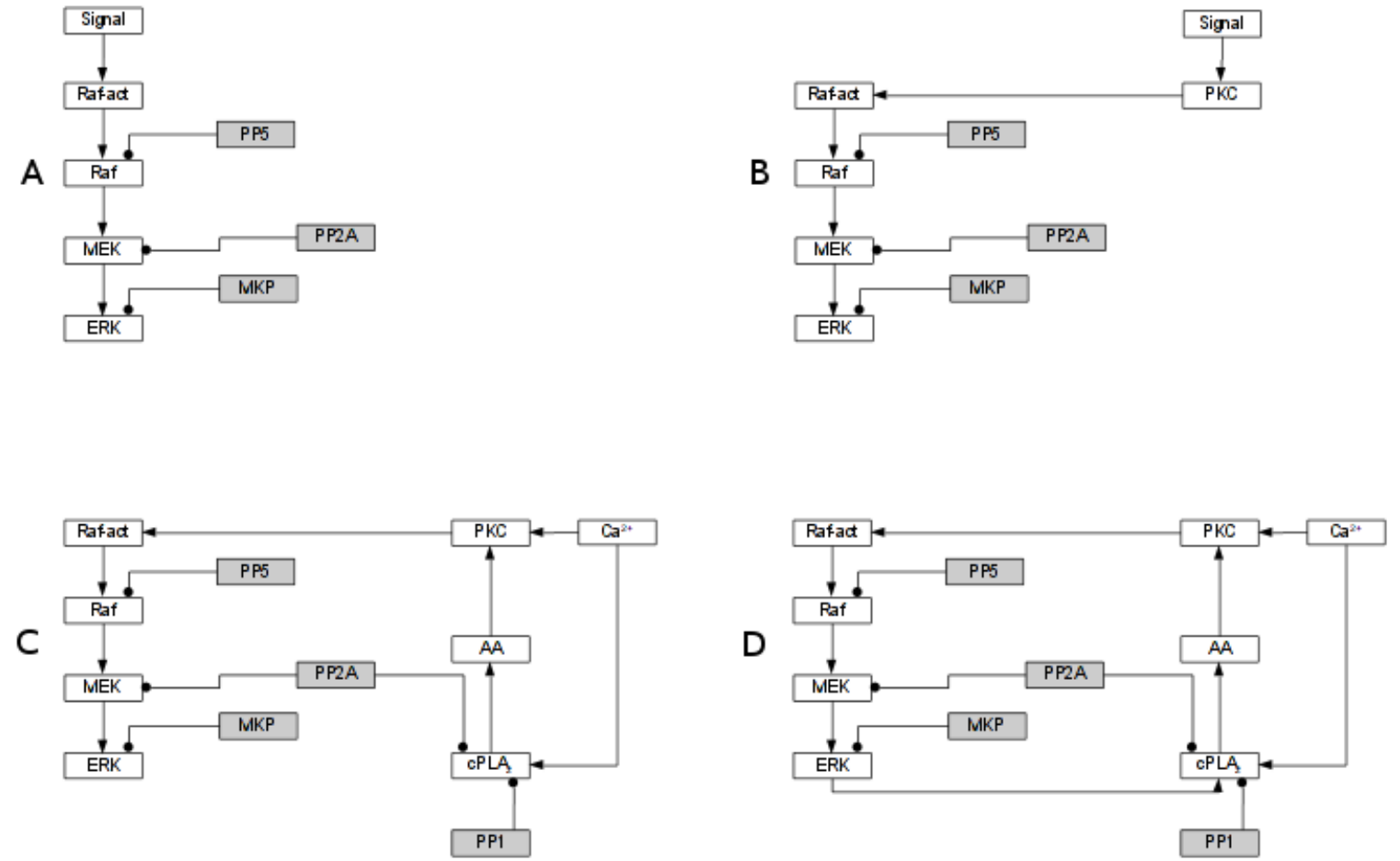

Figure 6.1: A possible sequence of steps to convert the pathway model into the network model. 
depicted in Figure 6.1. Beginning with the pathway model as it currently exists (part A), one could add PKC and apply the rate-based signal to it instead of Raf-act, as shown in part B; this would bring the upstream activation of the MAPK cascade into closer alignment. Next, one could add the molecules and reactions necessary for using calcium stimuli, which might require breaking the transition from part $\mathrm{B}$ to part $\mathrm{C}$ into two steps- perhaps first applying the $\mathrm{Ca}^{2+}$ input to $\mathrm{PKC}$ alone, and then adding $\mathrm{cPLA}_{2}, \mathrm{AA}$, and PP1. The last step would be to add the phosphorylation of cPLA $_{2}$ by ERK, thereby closing the positive feedback loop. One could then run a series of experiments similar to those used in this thesis to determine how each change contributes to the responses.

\subsubsection{Extending the Models and Methods}

Beyond the models described above, several extensions are envisaged. First, the known synaptic signal transduction network is considerably larger than the network used in this thesis. The PKA and CaMKII pathways, for instance, play important roles in the induction of LTP and LTD $[10,41,76]$, and should be included in future modelling work. Moreover, it is well-known that synaptic signalling networks are sensitive to temporal patterns of inputs and are capable of decoding these patterns to induce different forms of plasticity $[4,5,58,45]$; it would therefore be interesting to see how scaffolds affect this sensitivity. Finally, in terms of methods, all of the experiments performed in this thesis employed deterministic simulations, but several modelling studies have shown that properties and behaviours present in deterministic simulations can be quite different or absent altogether in stochastic simulations $[3$, 7, 8, 73]. Therefore, it is important that observed effects are eventually tested under 
both deterministic and stochastic conditions and results compared. 


\section{References}

[1] Ajay, S. M., and Bhalla, U. S. Synaptic Plasticity In Vitro and In Silico: Insights into an Intracellular Signaling Maze. Physiology 21, 4 (2006), 289-296.

[2] Alexa, A., Varga, J., And Reményi, A. Scaffolds are 'active' regulators of signaling modules. FEBS Journal 277, 21 (2010), 4376 - 4382.

[3] Antunes, G., and De Schutter, E. A Stochastic Signaling Network Mediates the Probabilistic Induction of Cerebellar Long-Term Depression. The Journal of Neuroscience 32, 27 (2012), 9288 - 9300.

[4] Bhalla, U. S. Biochemical Signaling Networks Decode Temporal Patterns of Synaptic Input. Journal of Computational Neuroscience 13 (2002), 49 - 62.

[5] Bhalla, U. S. Mechanisms for Temporal Tuning and Filtering by Postsynaptic Signaling Pathways. Biophysical Journal 83 (2002), 740 - 752.

[6] Bhalla, U. S. Understanding complex signaling networks through models and metaphors. Biophysics and Molecular Biology 81 (2003), 45 - 65.

[7] Bhalla, U. S. Signaling in Small Subcellular Volumes. I. Stochastic and Diffusion Effects on Individual Pathways. Biophysical Journal 87 (2004), 733 744 .

[8] Bhalla, U. S. Signaling in Small Subcellular Volumes. II. Stochastic and Diffusion Effects on Synaptic Network Properties. Biophysical Journal 87 (2004), $745-753$. 
[9] Bhalla, U. S. Still Looking for the Memories: Molecules and Synaptic Plasticity. In 20 Years of Computational Neuroscience, J. M. Bower, Ed., vol. 9 of Springer Series in Computational Neuroscience. Springer New York, 2013, pp. $187-205$.

[10] Bhalla, U. S., and Iyengar, R. Emergent Properties of Networks of Biological Signaling Pathways. Science 283 (1999), 381-387.

[11] Bhalla, U. S., And Iyengar, R. Robustness of the bistable behavior of a biological signaling feedback loop. Chaos: An Interdisciplinary Journal of Nonlinear Science 11, 1 (2001), 221-226.

[12] Bhalla, U. S., Ram, P. T., And Iyengar, R. MAP Kinase Phosphatase As a Locus of Flexibility in a Mitogen-Activated Protein Kinase Signaling Network. Science 297, 5583 (2002), 1018 - 1023.

[13] Blackwell, K. T., and Jedrzejewska-Szmek, J. Molecular mechanisms underlying neuronal synaptic plasticity: systems biology meets computational neuroscience in the wilds of synaptic plasticity. Wiley Interdisciplinary Reviews: Systems Biology and Medicine (2013), n/a.

[14] Blinov, M. L., Faeder, J. R., Golddstein, B., and Hlavacek, W. S. BioNetGen: software for rule-based modeling of signal transduction based on the interactions of molecular domains. Bioinformatics 20, 17 (2004), 3289 3291.

[15] Blüthgen, N., Legewie, S., Herzel, H., And Kholodenko, B. Mechanisms generating ultrasensitivity, bistability, and oscillations in signal transduction. In Introduction to Systems Biology, S. Choi, Ed. Humana Press, 2007, pp. 282-299.

[16] Boeckers, T. The postsynaptic density. Cell and Tissue Research 326, 2 (2006), 409-422.

[17] Brandman, O., And Meyer, T. Feedback loops shape cellular signals in space and time. Science 322 (2008), 390-395. 
[18] Bray, D. Signaling complexes: biophysical constraints on intracellular communication. Annual Review of Biophysics and Biomolecular Structure 27 (1998), $59-75$.

[19] Burke, J. E., And Dennis, E. A. Phospholipase A2 structure/function, mechanism, and signaling. Journal of Lipid Research 50 (2009), 237 - 242.

[20] Chan, C., Liu, X., Wang, L., Bardwell, L., Nie, Q., and Enciso, G. Protein scaffolds can enhance the bistability of multisite phosphorylation systems. PLoS Computational Biology 8, 6 (06 2012), e1002551.

[21] Chang, L., And Karin, M. Mammalian MAP kinase signalling cascades. Nature 410 (2001), $37-40$.

[22] Chen, W. W., Niepel, M., And Sorger, P. K. Classic and contemporary approaches to modeling biochemical reactions. Genes \&3 Development 24, 17 (2010), $1861-1875$.

[23] Chua, J. J. E., Kindler, S., Boyken, J., and Jahn, R. The architecture of an excitatory synapse. Journal of Cell Science 123, 6 (2010), 819-823.

[24] Citri, A., and Malenka, R. C. Synaptic plasticity: multiple forms, functions, and mechanisms. Neuropsychopharmacology 33, 1 (Jan. 2008), 18-41.

[25] Consortium, T. U. Activities at the universal protein resource (uniprot). Nucleic Acids Research 42, D1 (2014), D191-D198.

[26] Doi, T., Kuroda, S., Michikawa, T., and Kawato, M. Inositol 1,4,5Trisphosphate-Dependent Ca2+ Threshold Dynamics Detect Spike Timing in Cerebellar Purkinje Cells. The Journal of Neuroscience 25, 4 (2005), 950 - 961.

[27] England, J. L. Allostery in protein domains reflects a balance of steric and hydrophobic effects. Structure 19 (2011), 967 - 975.

[28] Faeder, J. R., Blinov, M. L., And Hlavacek, W. S. Rule-Based Modeling of Biochemical Systems with BioNetGen. In Systems Biology, I. V. Maly, Ed., vol. 500 of Methods in Molecular Biology. Humana Press, 2009, pp. 113 - 167. 
[29] Ferrell, J. E., and Bhatt, R. R. Mechanistic studies of the dual phosphorylation of mitogen-activated protein kinase. Journal of Biological Chemistry 272, 30 (1997), 19008 - 19016.

[30] Ferrell, J. E., And Xiong, W. Bistability in cell signaling: How to make continuous processes discontinuous, and reversible processes irreversible. Chaos: An Interdisciplinary Journal of Nonlinear Science 11 (2001), 227 - 236.

[31] Fisher, J., And Henzinger, T. A. Executable cell biology. Nature biotechnology 25, 11 (Nov. 2007), 1239-49.

[32] Good, M. C., Zalatan, J. G., And Lim, W. A. Scaffold Proteins: Hubs for Controlling the Flow of Cellular Information. Science 332 (2011), 680 - 686.

[33] Graupner, M., and Brunel, N. Mechanisms of induction and maintenance of spike-timing dependent plasticity in biophysical synapse models. Frontiers in Computational Neuroscience 4, 136 (2010).

[34] Hlavacek, W. S., Faeder, J. R., Blinov, M. L., Perelson, A. S., and Goldstein, B. The complexity of complexes in signal transduction. Biotechnology and Bioengineering 84, 7 (2003), 783 - 794.

[35] Hlavacek, W. S., Faeder, J. R., Blinov, M. L., Posner, R. G., Hucka, M., And Fontana, W. Rules for modeling signal-transduction systems. Science STKE 2006, 344 (2006), re6.

[36] Hu, J., Yu, H., Kornev, A. P., Zhao, J., Filbert, E. L., Taylor, S. S., AND SHAW, A. S. Mutation that blocks ATP binding creates a pseudokinase stabilizing the scaffolding function of kinase suppressor of Ras, CRAF and BRAF. Proceedings of the National Academy of Sciences 108, 15 (2011), 6067 - 6072.

[37] Johnson, G. L., and Lapadat, R. Mitogen-Activated Protein Kinase Pathways Mediated by ERK, JNK, and p38 Protein Kinases. Science 298, 5600 (2002), $1911-1912$.

[38] Jordan, J. D., Landau, E. M., and Iyengar, R. Signaling networks: the origins of cellular multitasking. Cell 103 (2000), 193-200. 
[39] Joyner, M. J. Giant sucking sound: can physiology fill the intellectual void left by the reductionists? Journal of Applied Physiology 111 (2011), 335 - 342.

[40] Karsenti, E. Self-organization in cell biology: a brief history. Nature Reviews Molecular Cell Biology 9 (2008), 255-262.

[41] Kawaguchi, S.-Y., And Hirano, T. Gating of long-term depression by $\mathrm{Ca} 2+$ /calmodulin-dependent protein kinaseII through enhanced cGMP signalling in cerebellar Purkinje cells. The Journal of Physiology 591, 7 (2013), $1707-1730$.

[42] Kennedy, M. B., Marcora, E., and Carlisle, H. J. Scaffold Proteins in the Postsynaptic Density. In Structural And Functional Organization Of The Synapse, J. Hell and M. Ehlers, Eds. Springer US, 2008, pp. 407-440.

[43] Kholodenko, B. N. Negative feedback and ultrasensitivity can bring about oscillations in the mitogen-activated protein kinase cascades. European Journal of Biochemistry 267, 6 (2000), 1583 - 1588.

[44] Kikuchi, S., Fujimoto, K., Kitagawa, N., Fuchikawa, T., Abe, M., Oka, K., TAKeI, K., And Tomita, M. Kinetic simulation of signal transduction system in hippocampal long-term potentiation with dynamic modeling of protein phosphatase 2A. Neural Networks 16 (2003), 1389 - 1398.

[45] Kim, M., Huang, T., Abel, T., and Blackwell, K. T. Temporal Sensitivity of Protein Kinase A Activation in Late-Phase Long Term Potentiation. PLoS Comput Biol 6, 2 (02 2010), e1000691.

[46] Kim, S. J., And Linden, D. J. Ubiquitous Plasticity and Memory Storage. Neuron 56, 4 (2007), $582-592$.

[47] Kitano, H. Systems biology: a brief overview. Science 295 (2002), $1662-1664$.

[48] Kitano, H. Biological robustness. Nature reviews. Genetics 5, 11 (Nov. 2004), $826-37$. 
[49] Kocieniewski, P., Faeder, J. R., And Lipniacki, T. The interplay of double phosphorylation and scaffolding in MAPK pathways. Journal of Theoretical Biology 295, 0 (2012), 116 - 124.

[50] Kohl, P., Crampin, E., Quinn, T., and Noble, D. Systems biology: an approach. Clinical Pharmacology 83 Therapeutics 88 (2010), 25 - 33.

[51] Kolch, W. Coordinating ERK/MAPK signalling through scaffolds and inhibitors. Nature Reviews Cell Biology 6 (2007), 827 - 837.

[52] Kolch, W., Calder, M., and Gilbert, D. When kinases meet mathematics: the systems biology of MAPK signalling. FEBS Letters 579, 8 (2005), 1891 1895. Systems Biology: Understanding the Biological Mosaic.

[53] Kotaleski, J. H., and Blackwell, K. T. Modelling the molecular mechanisms of synaptic plasticity using systems biology approaches. Nature reviews. Neuroscience 11, 4 (Apr. 2010), 239-251.

[54] Kuroda, S., Schweighofer, N., And Kawato, M. Exploration of signal transduction pathways in cerebellar long-term depression by kinetic simulation. The Journal of Neuroscience 21, 15 (2001), 5693 - 5702.

[55] Kyriakis, J., And Avruch, J. Mammalian mitogen-activated protein kinase signal transduction pathways activated by stress and inflammation. Physiological Reviews (2001).

[56] Letunic, I., Doerks, T., And Bork, P. Smart 7: recent updates to the protein domain annotation resource. Nucleic Acids Research 40, D1 (2012), D302 - D305.

[57] Levchenko, A., Bruck, J., And Sternberg, P. W. Scaffold proteins may biphasically affect the levels of mitogen-activated protein kinase signaling and reduce its threshold properties. Proceedings of the National Academy of Sciences 97, 11 (2000), 5818-5823. 
[58] Li, L., Stefan, M. I., And Le Novére, N. Calcium Input Frequency, Duration and Amplitude Differentially Modulate the Relative Activation of Calcineurin and CaMKII. PLoS ONE 7, 9 (09 2012), e43810.

[59] Locasale, J. W., and Chakraborty, A. K. Regulation of Signal Duration and the Statistical Dynamics of Kinase Activation by Scaffold Proteins. PLoS Computational Biology 4, 6 (06 2008), e1000099.

[60] Locasale, J. W., Shaw, A. S., And Chakraborty, A. K. Scaffold proteins confer diverse regulatory properties to protein kinase cascades. Proceedings of the National Academy of Sciences 104, 33 (2007), 13307 - 13312.

[61] Machado, D., Costa, R. S., Rocha, M., Ferreira, E. C., Tidor, B., AND Rocha, I. Modeling formalisms in Systems Biology. AMB Express 1, 1 (Jan. 2011), 45.

[62] Malenka, R. C., and Bear, M. F. LTP and LTD: An Embarrassment of Riches. Neuron 44 (2004), 5-21.

[63] Manninen, T., Hituri, K., Hellgren Kotaleski, J., Blackwell, K. T., AND Linne, M.-L. Postsynaptic signal transduction models for long-term potentiation and depression. Frontiers in Computational Neuroscience 4, 152 (2010), $1-29$.

[64] Markevich, N. I., Hoek, J. B., and Kholodenko, B. N. Signaling switches and bistability arising from multisite phosphorylation in protein kinase cascades. The Journal of Cell Biology 164, 3 (2004), 353 - 359.

[65] Mayer, B., Blinov, M., And Loew, L. Molecular machines or pleiomorphic ensembles: signaling complexes revisited. Journal of Biology 8, 9 (2009), 81.

[66] McKay, M. M., Ritt, D. A., And Morrison, D. K. Signaling dynamics of the ksr1 scaffold complex. Proceedings of the National Academy of Sciences 106, 27 (2009), $11022-11027$.

[67] Nelson, S. B., And Turrigiano, G. G. Strength through Diversity. Neuron 60, 3 (2008), $477-482$. 
[68] Newton, A. C. Protein kinase C: structural and spatial regulation by phosphorylation, cofactors, and macromolecular interactions. Chemical Reviews 101 (2001), $2353-2364$.

[69] Noble, D. Biophysics and systems biology. Philosophical Transactions of the Royal Society A 368, 1914 (Mar. 2010), 1125-39.

[70] Nurse, P. Life, logic and information. Nature 454 (2008), 424-426.

[71] Ogasamara, H., Doi, T., and Kawato, M. Systems Biology Perspectives on Cerebellar Long-Term Depression. Neurosignals 16 (2008), 300 - 317.

[72] Ogasawara, H., and Kawato, M. Bistable Switches for Synaptic Plasticity. Science Signaling 2 (2009), pe7.

[73] Ogasawara, H., and Kawato, M. Computational Models of Cerebellar Long-Term Memory. In Systems Biology, S. Nakanishi, R. Kageyama, and D. Watanabe, Eds. Springer Japan, 2009, pp. 169-181.

[74] Ogasawara, H., and Kawato, M. The protein kinase mzeta network as a bistable switch to store neuronal memory. BMC Systems Biology 4, 1 (2010), 181.

[75] Okabe, S. Molecular anatomy of the postsynaptic density. Molecular and Cellular Neuroscience 34, 4 (2007), $503-518$.

[76] Oliveira, R. F., Kim, M., and Blackwell, K. T. Subcellular Location of PKA Controls Striatal Plasticity: Stochastic Simulations in Spiny Dendrites. PLoS Comput Biol 8, 2 (02 2012), e1002383.

[77] OMalley, M. A., And Dupré, J. Fundamental issues in systems biology. BioEssays (2005).

[78] Pi, H. J., And Lisman, J. E. Coupled Phosphatase and Kinase Switches Produce the Tristability Required for Long-Term Potentiation and Long-Term Depression. The Journal of Neuroscience 28, 49 (2008), 13132 - 13138. 
[79] Ramakrishnan, N., and Bhalla, U. S. Memory switches in chemical reaction space. PLoS Computational Biology 4, 7 (07 2008), e1000122.

[80] Raman, M., Chen, W., And Cobb, M. H. Differential regulation and properties of MAPKs. Oncogene 26 (2007), 3100 - 3112.

[81] Robertson, M. Ockham's broom: A new series. Journal of Biology 8, 9 (2009), 79.

[82] Sekar, J. A. P., And Faeder, J. R. Rule-Based Modeling of Signal Transduction: A Primer. In Computational Modeling of Signaling Networks, X. Liu and M. D. Betterton, Eds., vol. 880 of Methods in Molecular Biology. Humana Press, 2012, pp. $139-218$.

[83] Sheng, M., And Kim, E. The Postsynaptic Organization of Synapses. Cold Spring Harbor Perspectives in Biology 3, 12 (2011), a005678.

[84] Smith, A., Xu, W., Sun, Y., Faeder, J., and Marai, G. E. RuleBender: integrated modeling, simulation and visualization for rule-based intracellular biochemistry. BMC Bioinformatics 13, Suppl 8 (2012), S3.

[85] Smith, F. D., And Scott, J. D. Signaling complexes: Junctions on the intracellular information super highway. Current Biology 12 (2001), R32 - R40.

[86] Smock, R. G., and Gierasch, L. M. Sending signals dynamically. Science 324 (2009), $198-203$.

[87] Smolen, P., Baxter, D. A., and Byrne, J. H. Bistable MAP kinase activity: a plausible mechanism contributing to maintenance of late long-term potentiation. American Journal of Physiology - Cell Physiology 294, 2 (2008), C503 - C515.

[88] Smolen, P., Baxter, D. A., And Byrne, J. H. Interlinked dual-time feedback loops can enhance robustness to stochasticity and persistence of memory. Phys. Rev. E 79 (Mar 2009), 031902. 
[89] Specht, C. G., And Triller, A. The dynamics of synaptic scaffolds. BioEssays 30, 11-12 (2008), $1062-1074$.

[90] Tanaka, K., And Augustine, G. Systems Biology Meets Single-Cell Physiology: Role of a Positive-Feedback Signal Transduction Network in Cerebellar Long-Term Synaptic Depression. In Systems Biology, S. Nakanishi, R. Kageyama, and D. Watanabe, Eds. Springer Japan, 2009, pp. 159-168.

[91] Tanaka, K., And Augustine, G. J. A positive feedback signal transduction loop determines timing of cerebellar long-term depression. Neuron 59, 4 (2008), $608-620$.

[92] Tanaka, K., Khiroug, L., Santamaria, F., Doi, T., Ogasawara, H., Ellis-Davies, G., Kawato, M., and Augustine, G. J. Ca2+ Requirements for Cerebellar Long-Term Synaptic Depression: Role for a Postsynaptic Leaky Integrator. Neuron 54, 5 (2007), $787-800$.

[93] Thalhauser, C. J., And Komarova, N. L. Signal Response Sensitivity in the Yeast Mitogen-Activated Protein Kinase Cascade. PLoS ONE 5, 7 (07 2010), e11568.

[94] Tracy, T. S., And Hummel, M. A. Modeling kinetic data from in vitro drug metabolism enzyme experiments. Drug Metabolism Reviews 36, 2 (2004), 231 242. PMID: 15237853 .

[95] Witzel, F., Maddison, L. E., And Blthgen, N. How Scaffolds shape MAPK signalling: What we know and opportunities for systems approaches. Frontiers in Physiology 3, 475 (2013).

[96] Yang, J., And HlavaceK, W. S. Scaffold-mediated nucleation of protein signaling complexes: Elementary principles. Mathematical Biosciences 232, 2 (2011), $164-173$.

[97] Zeke, A., Lukacs, M., Lim, W. A., And Remenyi, A. Scaffolds: interaction platforms for cellular signalling circuits. Trends in Cell Biology 19 (2009), 364374. 


\section{Appendix A}

\section{Validation of the Synaptic Model}

This Appendix presents the procedure we used to validate our implementation of the Antunes and De Schutter model of cerebellar LTD. First, it should be noted that Antunes and De Schutter validated their model [3] against the experimental results reported by Tanaka et al. in [92]. Therefore, to validate our implementation, we followed the same procedure that Antunes and De Schutter reported in [3].

Before describing the validation process, we should note some important differences between the simulations used for validation and those used in the experiments in the main text. First, we measured different output molecules as indicators of the induction of plasticity. For validation, we measured the concentration of AMPA receptors present at the synapse, because that is the measure used in the original model; for the experiments, we measured the concentration of activated ERK, because we wanted to compare our network and pathway models and the pathway model does not include AMPA receptors. Second, for validation, we used stochastic simulations and averaged the results of 80 runs - again, because this was the procedure in the original. For the experiments, we used deterministic simulations because they allowed us to explore parameter space of the models more efficiently without the need for averaging responses.

Antunes and De Schutter identified several experimental results that their model needed to reproduce for validation. We used the simulation results they reported in [3] as the criteria for validation of our model. We list these criteria below and reference the figures that follow for evidence that our model satisfied them. 
1. At rest, the mean number of synaptic AMPARs in the model should be between 120 and 130; this is evident in Figure A.1 before the delivery of the calcium pulse at 600 seconds

2. Following delivery of the stimulus, the number of synaptic AMPARs in the model should reach a stable state within approximately 20 minutes (1200 seconds); this is evident Figure A.1, since the traces reach a stable state by 1800 seconds (i.e., 1200 seconds post-stimulus)

3. The model should produce the sigmoidal relationship between the concentration of the calcium stimulus and the magnitude of LTD described by Equation A.1. This is evident in Figure A.2; the curves were obtained by nonlinear regression to Equation A.1. The parameters are: the Hill coefficient $(n)$, the $\left[\mathrm{Ca}^{2+}\right]$ required to achieve the half-maximum magnitude of $\operatorname{LTD}(K)$, and the maximum magnitude of LTD $\left(L T D_{\max }\right)$. For each curve, these parameters were estimated with $95 \%$ confidence intervals

4. Increasing the duration of the input calcium pulse should decrease the threshold required for induction of LTD; this is evident in the leftward shift of the curves in Figure A.2 with increasing duration of calcium stimulus, and in the decrease of $K$ with increasing duration in Figure A.3

5. Induction of LTD by $\mathrm{Ca}^{2+}$ should be highly cooperative with Hill coefficients between 5 and 7; this is evident in Figure A.4

6. The maximum magnitude of LTD should be independent of stimulus duration; this is evident in Figure A.5

$$
L T D=L T D_{\max }\left(\frac{\left[C a^{2+}\right]^{n}}{K^{n}+\left[C a^{2+}\right]^{n}}\right)
$$




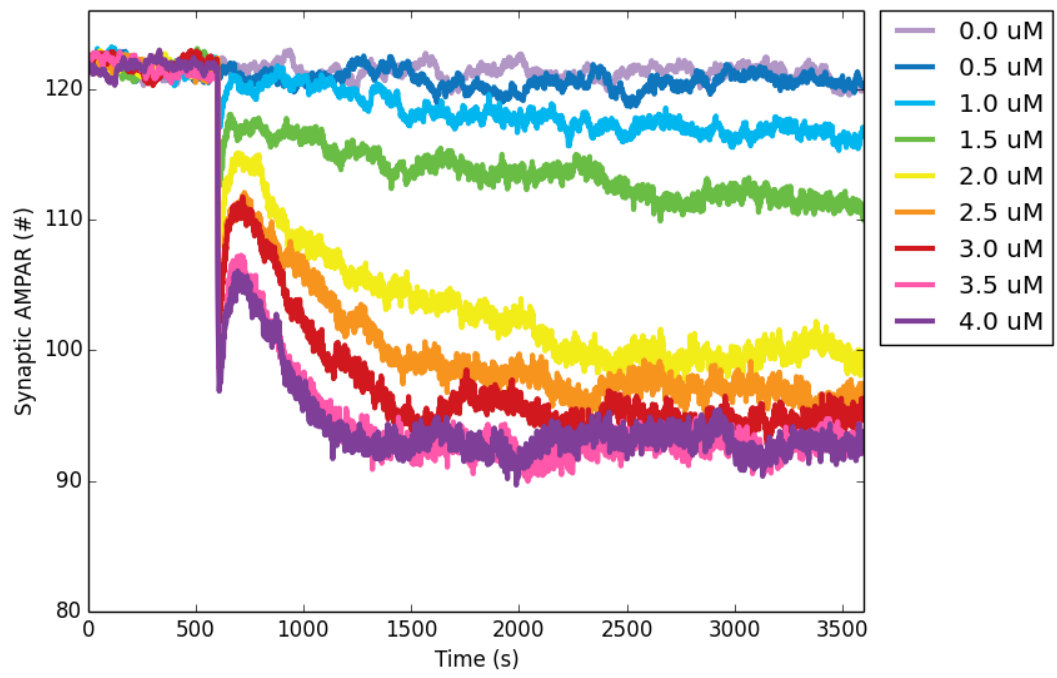

Figure A.1: Mean number of AMPA receptors in the synapse following stimulation with 1-second pulses of $\mathrm{Ca}^{2+}$ at different concentrations

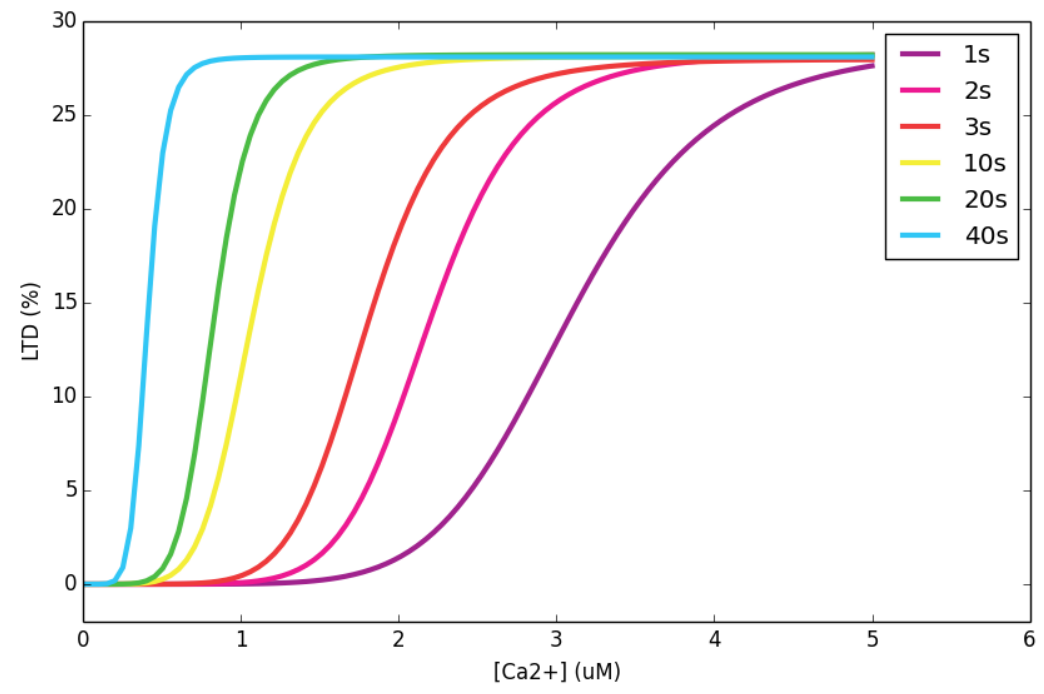

Figure A.2: Sigmoidal relationships between concentration of calcium stimulus and magnitude of LTD. Curves were obtained by nonlinear regression to Equation A.1 


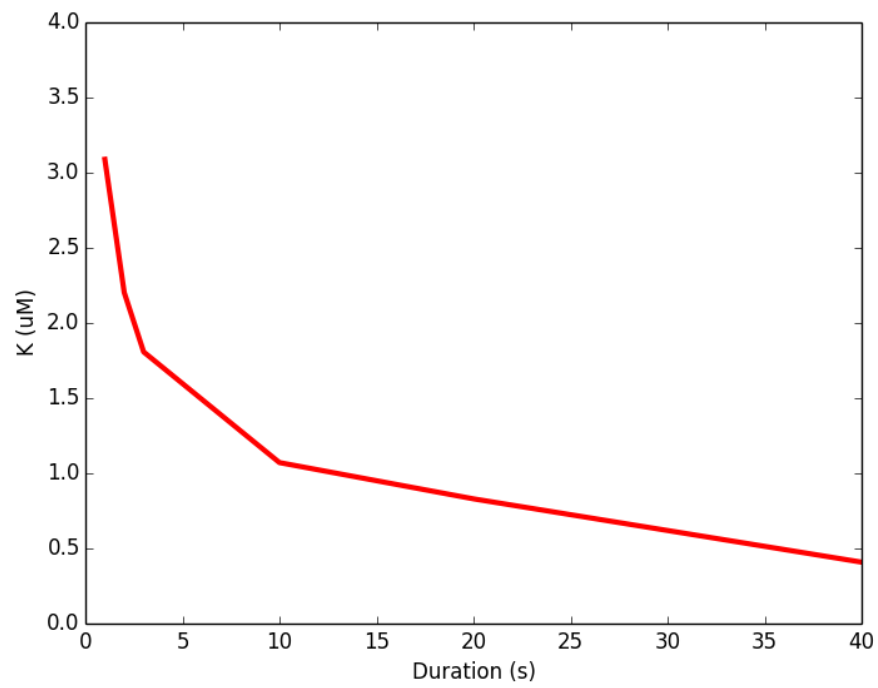

Figure A.3: Estimated values of $K$ (the $\mathrm{Ca}^{2+}$ input required for induction of LTD) at different durations of stimulation



Figure A.4: Estimated values of $n$ (the Hill coefficient) at different durations of stimulation 


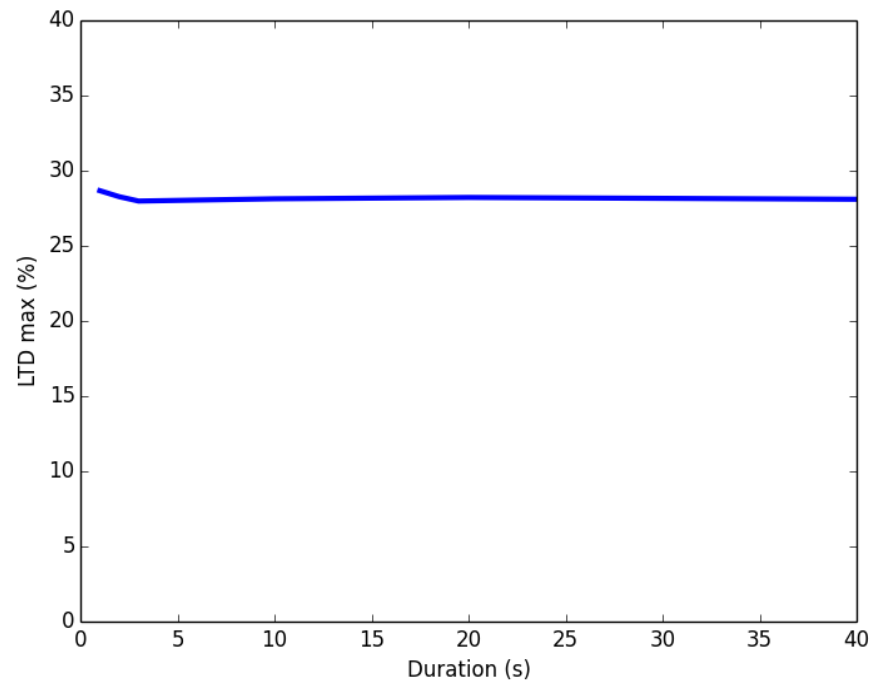

Figure A.5: Estimated values of $L T D_{\max }$ (the maximum magnitude of LTD) at different durations of stimulation 


\section{Appendix B}

\section{Supplementary Experimentation}

In this Appendix, we provide results of additional experiments performed on the pathway and network models. The charts in the following sections are from early tests in which we varied different parameters from the ones used in the main experiments. Note that all of the results here are for variations of scaffold parameters, specifically the association rate constant $(k f)$, the catalytic rate constant for enzymatic reactions on the scaffold (Pscaff), and scaffold concentration ([KSR]). These tests generated a large number of plots; rather than presenting them all as two-dimensional curves, we have summarized the results in bar charts. For each experiment, we varied one of the above parameters over a certain range (indicated in the tables below) at three different stimulus magnitudes. The charts display the ERK response to the initial and final values of the parameter in question as well as the peak ERK response. The value of the peak ERK response relative to the initial and final responses provides a rough indication of the shape of the underlying curve. For instance, in experiments that varied $[K S R]$, the initial and final ERK responses are much lower than the peak response, which indicates the typical biphasic curve for scaffold concentration. This approach does not supply any information about the scaffold concentration that corresponds to the peak ERK response, but it does provide a general sense of a variety of results at a glance. We should also note that these charts include experiments with the distributive versions of our models for reference. It is interesting to note that the only situation in which the distributive model responses are as strong as those of the processive models is when the association rate is varied. Summary 


\begin{tabular}{|l|l|l|}
\hline Parameter & Initial & Final \\
\hline \hline$[K S R]$ & $1 \mathrm{e}-3 \mu \mathrm{M}$ & $10.0 \mu \mathrm{M}$ \\
\hline Pscaff & $0.0 s^{-1}$ & $50.0 s^{-1}$ \\
\hline$k f$ & $0.0 s^{-1}$ & $50.0 s^{-1}$ \\
\hline
\end{tabular}

Table B.1: Parameter ranges for additional experiments on the pathway and network models.

charts for supplementary experiments on the pathway and network models follow. All experiments use the parameters and ranges in Table B. Figures B.1, B.2, and B.3 show the results of pathway experiments without feedback, with positive feedback, and with negative feedback, respectively. Figures B.4, B.5, and B.6 show the results of network experiments with default feedback, positive feedback, and negative feedback, respectively. 




Figure B.1: Summary charts for supplementary experiments on the pathway model without feedback. 


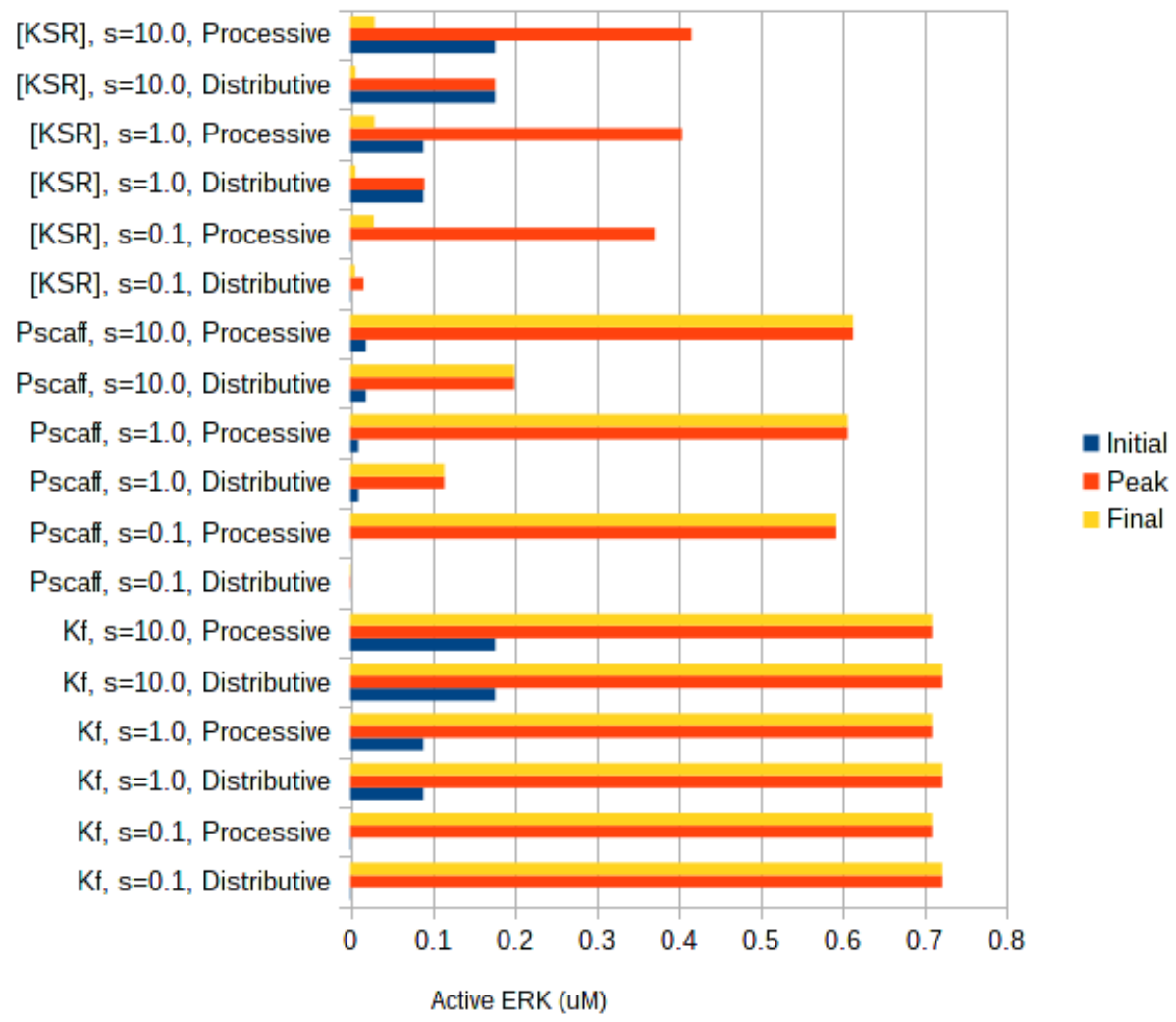

Figure B.2: Summary charts for supplementary experiments on the pathway model with positive feedback. 


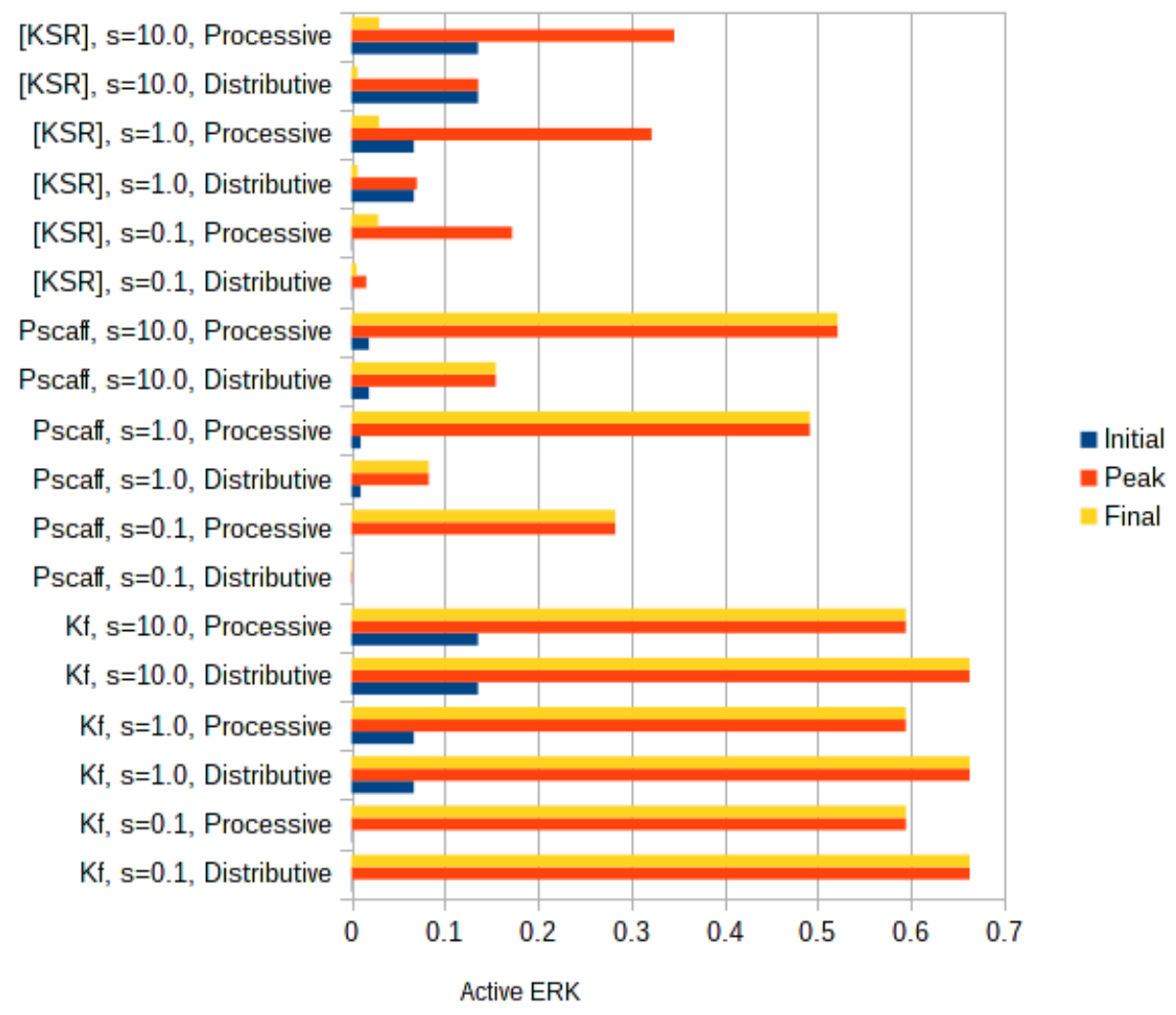

Figure B.3: Summary charts for supplementary experiments on the pathway model with negative feedback. 


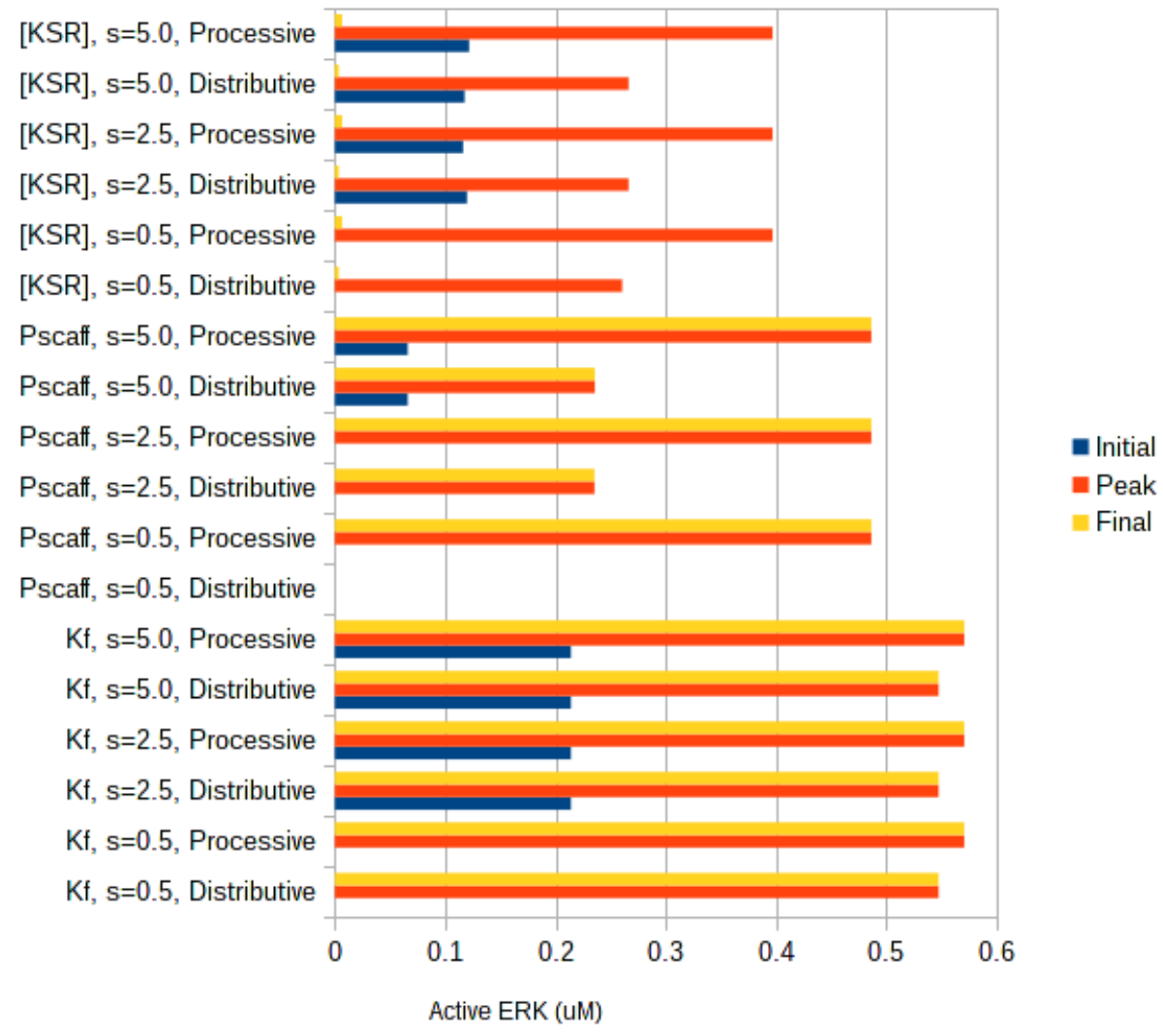

Figure B.4: Summary charts for supplementary experiments on the network model default feedback. 


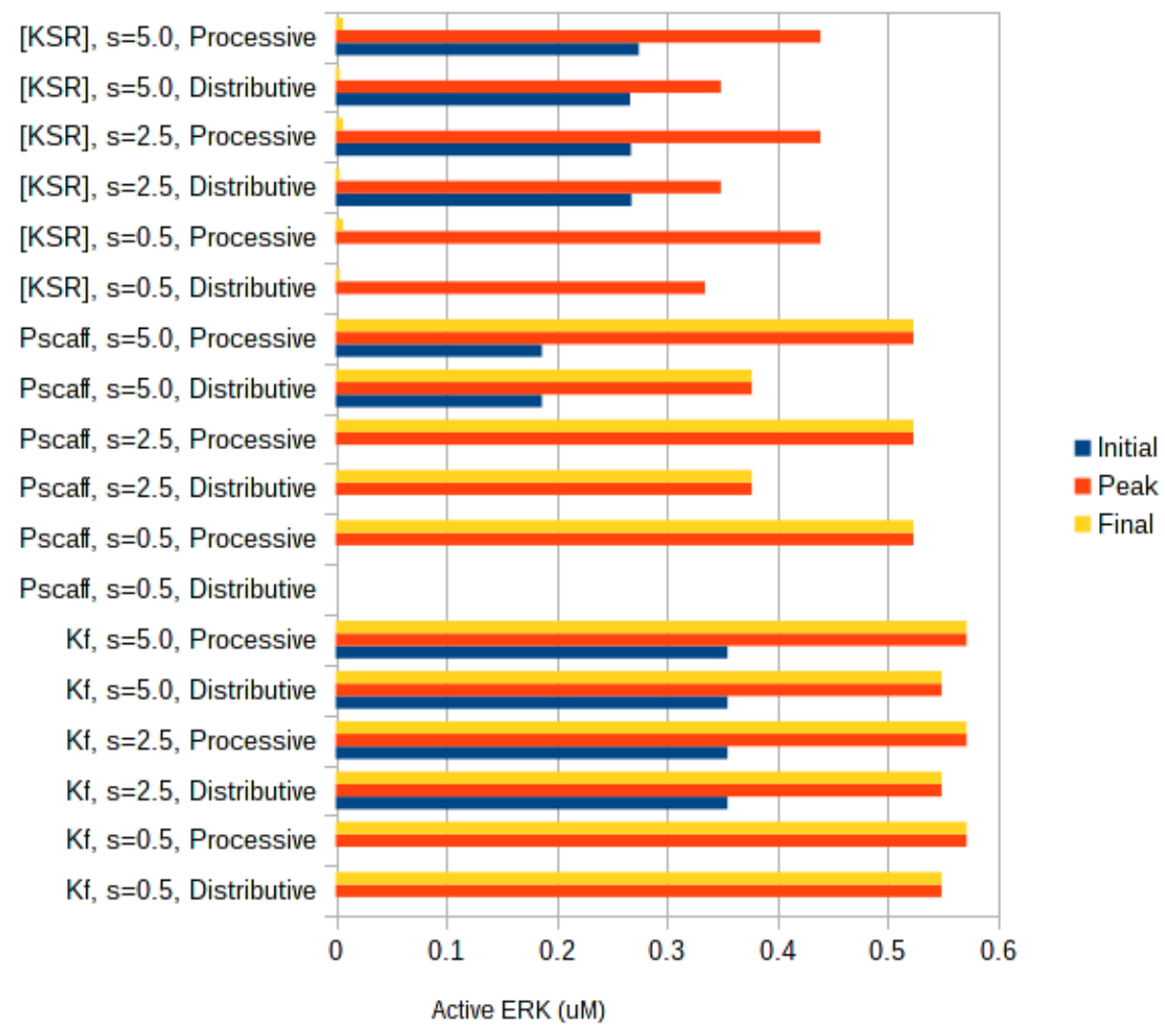

Figure B.5: Summary charts for supplementary experiments on the network model with positive feedback. 


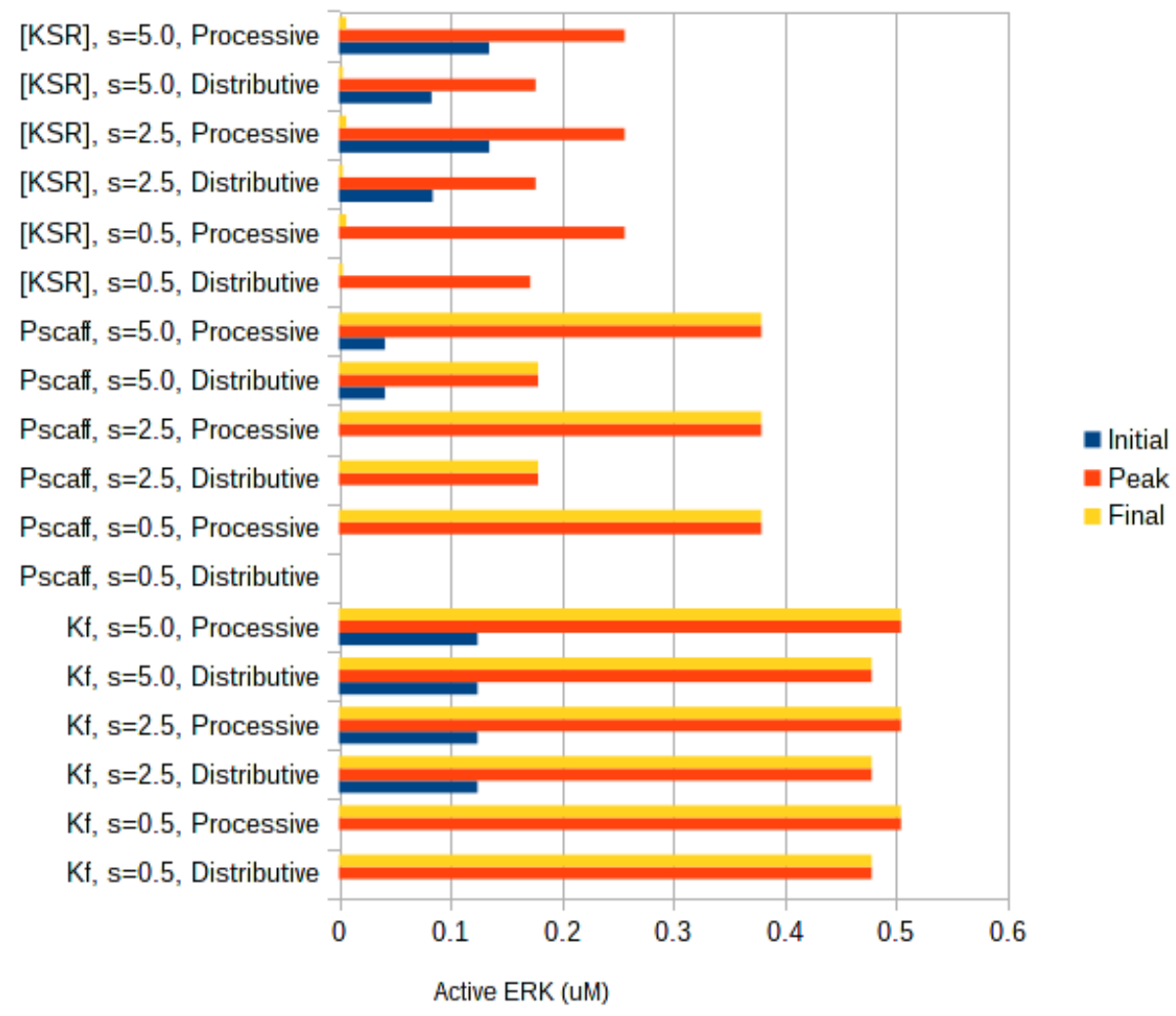

Figure B.6: Summary charts for supplementary experiments on the network model with negative feedback. 